\title{
Pd-Catalyzed and Copper Assisted Regioselective Sequential C2 and C7 Arylation of Thiazolo[5,4-f]quinazolin-9(8H)-one with Aryl Halides.
}

Marine Harari, Florence Couly, Corinne Fruit,* Thierry Besson.*

Normandie Univ, UNIROUEN, INSA Rouen, CNRS, COBRA,76000 Rouen, France.

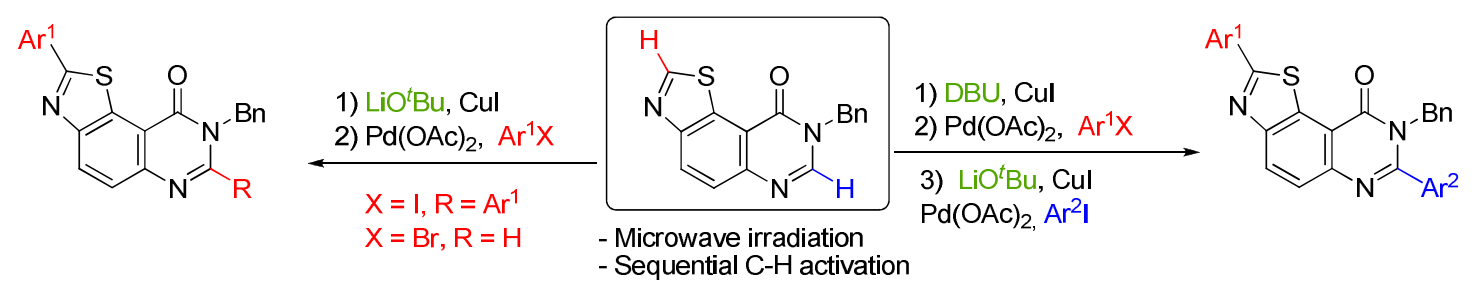




\section{Contents}

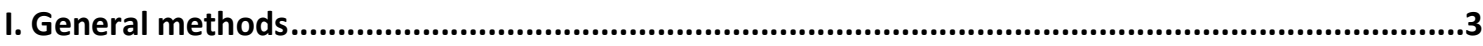

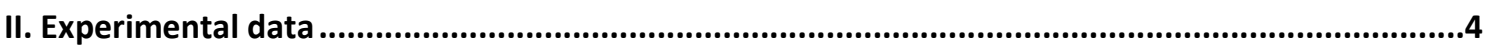

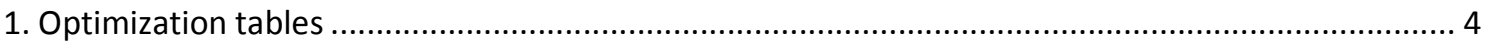

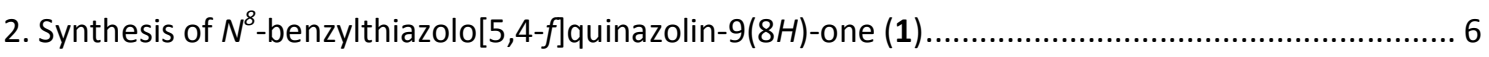

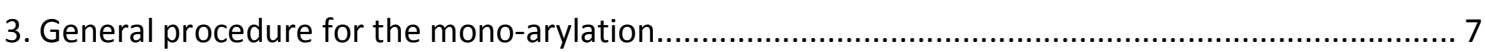

$N^{8}$-benzyl-2-phenylthiazolo[5,4-f]quinazolin-9(8H)-one (2a) ...................................................... 7

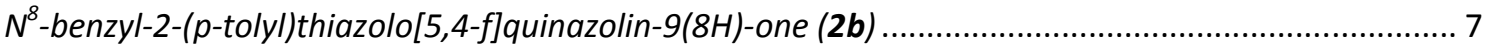

$N^{8}$-benzyl-2-(4-methoxyphenyl)thiazolo[5,4-f]quinazolin-9(8H)-one (2c) ...................................... 8

$N^{8}$-benzyl-2-(4-chlorophenyl)thiazolo[5,4-f]quinazolin-9(8H)-one (2d) ....................................... 8

$N^{8}$-benzyl-2-(4-fluorophenyl)thiazolo[5,4-f]quinazolin-9(8H)-one (2e).......................................... 9

4-( $N^{8}$-benzyl-9-oxo-8,9-dihydrothiazolo[5,4-f]quinazolin-2-yl)benzonitrile (2f)............................... 9

$N^{8}$-benzyl-2-(2,4-dichlorophenyl)thiazolo[5,4-f]quinazolin-9(8H)-one (2g) ...................................... 9

$N^{8}$-benzyl-2-(4-(dimethylamino)phenyl)thiazolo[5,4-f]quinazolin-9(8H)-one (2h).............................. 10

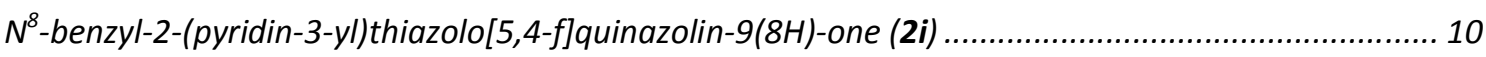

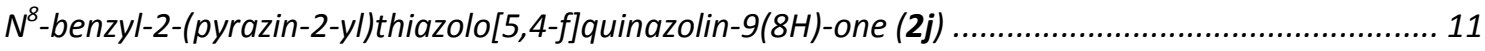

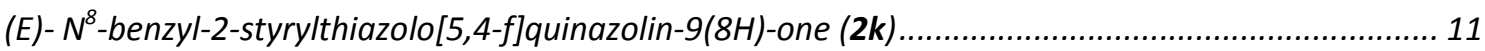

4. General procedure for the bis-arylation ......................................................................... 11

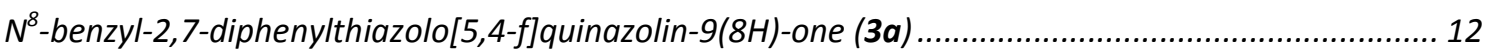

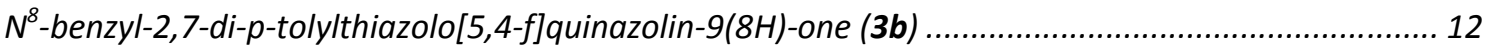

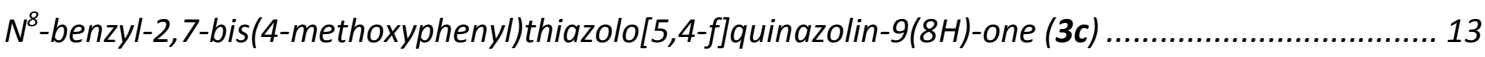

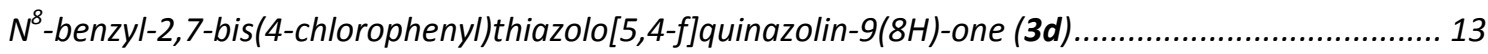

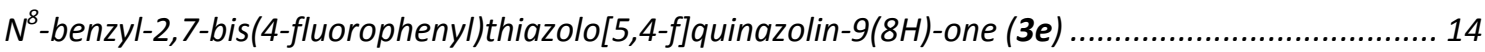

4,4'-( N'-benzyl-9-oxo-8,9-dihydrothiazolo[5,4-f]quinazoline-2,7-diyl)dibenzonitrile (3f) .................. 14

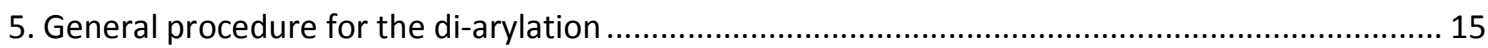

$N^{8}$-benzyl-2-phenyl-7-(p-tolyl)thiazolo[5,4-f]quinazolin-9(8H)-one (4a) ..................................... 15

4-( $N^{8}$-benzyl-9-oxo-2-(p-tolyl)-8,9-dihydrothiazolo[5,4-f]quinazolin-7-yl)benzonitrile (4b)................. 16

4-( $N^{8}$-benzyl-2-(4-methoxyphenyl)-9-oxo-8,9-dihydrothiazolo[5,4-f]quinazolin-7-yl)benzonitrile(4ca) 16

$N^{8}$-benzyl-7-(4-fluorophenyl)-2-(4-methoxyphenyl)thiazolo[5,4-f]quinazolin-9(8H)-one (4cb) ............ 17

4-( $N^{8}$-benzyl-2-(4-chlorophenyl)-9-oxo-8,9-dihydrothiazolo[5,4-f]quinazolin-7-yl)benzonitrile (4da).. 17 $N^{8}$-benzyl-2-(4-chlorophenyl)-7-(p-tolyl)thiazolo[5,4-f]quinazolin-9(8H)-one (4db) ............................. 18

$N^{8}$-benzyl-2-(4-chlorophenyl)-7-(4-methoxyphenyl)thiazolo[5,4-f]quinazolin-9(8H)-one (4dc)........... 18

$N^{8}$-benzyl-2-(4-fluorophenyl)-7-(p-tolyl)thiazolo[5,4-f]quinazolin-9(8H)-one (4e)............................... 19

4-( $N^{8}$-benzyl-7-(4-fluorophenyl)-9-oxo-8,9-dihydrothiazolo[5,4-f]quinazolin-2-yl)benzonitrile (4fa) ... 19

4-( $N^{8}$-benzyl-9-oxo-7-(o-tolyl)-8,9-dihydrothiazolo[5,4-f]quinazolin-2-yl)benzonitrile (4fb) ................ 20

6. General one-pot procedure for the sequential C-H bis-arylation ................................................... 20

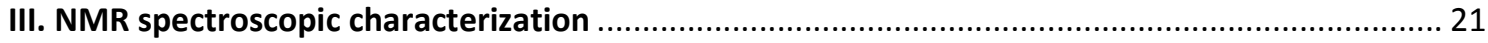




\section{General methods}

Materials were obtained commercially and used without further purification. All reactions were carried out under inert atmosphere of argon or nitrogen and monitored by thin-layer chromatography with silica gel 60 F254 pre-coated aluminium plates $(0,25 \mathrm{~mm})$. Visualization was performed with a UV light at 254 and $312 \mathrm{~nm}$.

Purifications were carried out by flash column chromatography system equipped with a dual UV-Vis spectrophotometer (200-600 nm), a fraction collector (176 tubes), a dual piston pump (1 to $200 \mathrm{~mL} / \mathrm{min}, \mathrm{P}_{\max }=15$ bar) allowing quaternary gradients and an additional inlet for air purge. Samples can be injected in liquid or solid mode.

Melting points of solid compounds were measured on a STUART Melting Point SMP3 with a precision of $+/-1.5{ }^{\circ} \mathrm{C}$. IR spectra were recorded on a PerkinElmer Spectrum 100 Series FT-IR spectrometer.

Liquids and solids were applied on the Single Reflection Attenuated Total Reflectance (ATR) Accessories. Absorption bands are given in $\mathrm{cm}^{-1}$.

${ }^{1} \mathrm{H},{ }^{13} \mathrm{C}\left\{{ }^{1} \mathrm{H}\right\}$ NMR spectra were recorded at $295^{\circ} \mathrm{K}$ on a Bruker AVANCE $300 \mathrm{MHz}$, at 300 and $75.4 \mathrm{MHz}$ respectively. Abbreviations used for peak multiplicities are s: singlet, $\mathrm{d}$ : doublet, t: triplet, q: quadruplet and $\mathrm{m}$ : multiplet. Coupling constants $J$ are in $\mathrm{Hz}$ and chemical shifts are given in $\mathrm{ppm}\left(\delta_{H}\right.$ and $\left.\delta_{C}\right)$ relative to $\mathrm{CDCl}_{3}$ (residual solvent signals).

Mass spectra analysis was performed by the Mass Spectrometry Laboratory of the University of Rouen. Mass spectra (ESI) were recorded with a Waters LCP 1er XR spectrometer.

Microwave experiments were conducted in a commercial microwave reactor especially designed for synthetic chemistry. Experiments were conducted in two different microwave reactors (Anton Paar Monomode $300^{\mathrm{TM}}$ and Biotage Initiator ${ }^{+}$) both equipped with a monomode cavity with a microwave power delivery system ranging from 0 to $850 \mathrm{~W}$ allowing pressurized reactions ( 0 to 30 bars) to be carried out in sealed glass vials ( 0.5 to 20 $\mathrm{mL}$ ) equipped with a snap cap and a silicon septum. The temperature $\left(0\right.$ to $\left.300{ }^{\circ} \mathrm{C}\right)$ was monitored via a contact-less infrared sensor and was calibrated with a Ruby Thermometer. Temperature, pressure, and power profiles were edited and monitored through a touch screen control panel. Time indicated in the various protocols is the time measured when the mixtures reached the programmed temperature after a ramp period of $2 \mathrm{~min}$. 


\section{Experimental data}

\section{Optimization tables}

Table 1. $\mathrm{C} 2-\mathrm{H}$ arylation of $N^{8}$-benzylated-thiazolo[5,4-f]quinazolin-9(8H)-one 1 with copper iodide $^{a}$.<smiles></smiles>

1

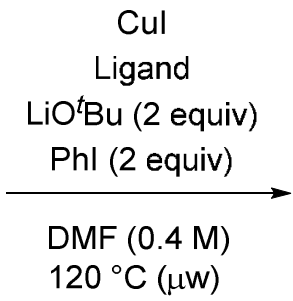<smiles>O=c1c2c(ccc3nc(-c4ccccc4)sc32)ncn1Cc1ccccc1</smiles>

$2 a$

\begin{tabular}{|c|c|c|c|c|c|}
\hline entry & $\begin{array}{l}\text { metal loading } \\
\text { (mol\%) }\end{array}$ & time (min) & ligand (mol\%) & 2a (yield \%) & 1 (yield \%) \\
\hline 1 & 10 & 10 & - & 28 & 66 \\
\hline 2 & 10 & 30 & - & 41 & 51 \\
\hline 3 & 30 & 30 & - & 40 & 55 \\
\hline 4 & 100 & 30 & - & 38 & 57 \\
\hline 5 & 10 & 60 & Phenanthroline (10) & 21 & 77 \\
\hline 6 & 10 & 60 & $\mathrm{PPh}_{3}(10)$ & 15 & 81 \\
\hline 7 & 10 & 240 & - & 62 & 31 \\
\hline $8^{c}$ & 100 & 240 & Phenanthroline (10) & 0 & 100 \\
\hline
\end{tabular}


Table 2. $\mathrm{C} 2-\mathrm{H}$ arylation of $N^{8}$-benzylated-thiazolo[5,4-f]quinazolin-9(8H)-one 1 with DBU as base $^{a}$.<smiles>O=c1[nH]c(Cc2ccccc2)cc2ccc3nc([TlH])sc3c12</smiles>

1

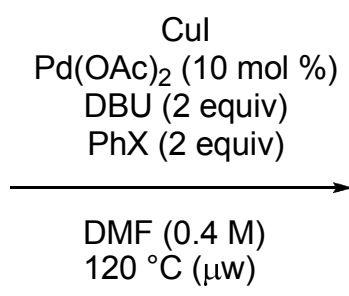

$120^{\circ} \mathrm{C}(\mu \mathrm{w})$<smiles></smiles>

2a

$\begin{array}{ccccccc}\text { entry } & \text { Cul loading (mol\%) } & \mathrm{PhX} & \text { heating } & \text { time }(\mathrm{h}) & \mathbf{2 a}(\text { yield \%) } & \mathbf{1} \text { (yield } \%) \\ 1 & 1 \text { equiv } & \mathrm{Phl} & \text { traditional } & 7 & 92 & 0 \\ 2 & - & \mathrm{Phl} & \text { traditional } & 7 & 0 & 100 \\ 3 & 10 & \mathrm{Phl} & \text { traditional } & 7 & 14 & 79 \\ 4 & 50 & \mathrm{Phl} & \text { traditional } & 7 & 31 & 57 \\ 5^{c} & 1 \text { equiv } & \mathrm{Phl} & \text { traditional } & 7 & 76 & 18 \\ 6 & 1 \text { equiv } & \mathrm{Phl} & \mu \mathrm{w} & 1,5 & 57 & 38 \\ 7 & 1 \text { equiv } & \mathrm{Phl} & \mu \mathrm{w} & 3 & 87 & 0 \\ 8^{d} & 1 \text { equiv } & \mathrm{Phl} & \mu \mathrm{w} & 3 & 38 & 57 \\ 9 & 1 \text { equiv } & \mathrm{PhBr} & \mu \mathrm{w} & 3 & 50 & 47 \\ 10 & 1 \text { equiv } & \mathrm{PhBr} & \mu \mathrm{w} & 4 & 76 & 18 \\ 11 & 1 \text { equiv } & \mathrm{PhBr} & \mu \mathrm{w} & 5 & 92 & 0\end{array}$

${ }^{a}$ Conditions: reactions were performed in a sealed tube at $0.4 \mathrm{M}$ with $\mathrm{Cul}$ ( $\mathrm{x} \mathrm{mol} \%$ ), DBU (2 equiv) and 1 (1 equiv) in a microwave reactor, before adding $\mathrm{PhX}\left(2\right.$ equiv) and $\mathrm{Pd}(\mathrm{OAc})_{2}(10 \mathrm{~mol} \%) .{ }^{b}$ Reported yields are isolated yields. ${ }^{c}$ Reaction was performed with $5 \mathrm{~mol} \%$ of $\mathrm{Pd}(\mathrm{OAc})_{2} .{ }^{d}$ Reaction was performed with 1 equiv of DBU. 
2. Synthesis of $N^{8}$-benzylthiazolo[5,4-f]quinazolin-9(8H)-one $(1)^{1}$<smiles>Nc1ccc([N+](=O)[O-])cc1C(=O)O</smiles><smiles>O=c1[nH]cnc2ccc([N+](=O)[O-])cc12</smiles><smiles></smiles><smiles>Nc1ccc2ncn(Br)c(=O)c2c1</smiles><smiles></smiles><smiles></smiles><smiles>C1CCCCC1</smiles>

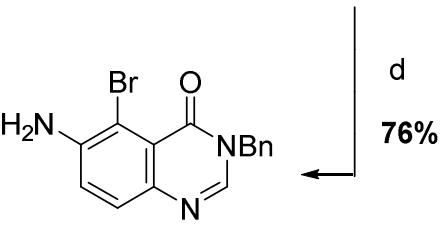

a) DMA-DMF (2.5 equiv), AcOEt (1 M), $30 \mathrm{~min}, 77^{\circ} \mathrm{C}(\mu \mathrm{w}) ;$ b) $\mathrm{H}_{2} \mathrm{NBn}$ (1.5 equiv), AcOH (1 M), $30 \mathrm{~min}, 118^{\circ} \mathrm{C}$ $(\mu \mathrm{w}) ; \mathrm{c}) \mathrm{Pd} / \mathrm{C} 10 \%(10 \% \mathrm{w} / \mathrm{w}), \mathrm{NH}_{4} \mathrm{CO}_{2} \mathrm{H}$ (5 equiv), EtOH (0.3 M), $30 \mathrm{~min}, 78^{\circ} \mathrm{C}(\mu \mathrm{w})$; d) NBS (1,1 equiv), DMF $(0.1 \mathrm{M}), 2 \mathrm{~h}$, r.t.; e) 4,5-Dichloro-1,2,3-dithiazolium chloride (Appel salt, 1.3 equiv), pyridine (2 equiv), DCM (0.3 M), 3 h, r.t.; f) Cul (1 equiv), pyridine ( $0.3 \mathrm{M}), 30 \mathrm{~min}, 115^{\circ} \mathrm{C}(\mu \mathrm{w})$.

$N^{8}$-benzylthiazolo[5,4-f]quinazolin-9(8H)-one (1)<smiles></smiles>

$N^{8}$-benzyl-9-oxo-8,9-dihydrothiazolo[5,4-f] quinazoline-2-carbonitrile (3 g, $9.424 \mathrm{mmol}, 1$ equiv) in $\mathrm{HBr}(0.3 \mathrm{M})$ is stirred under microwave irradiation in an open vessel for $1 \mathrm{~h}$. The resulting solution is diluted with dichloromethane and neutralized with an aqueous saturated solution of $\mathrm{NaHCO}_{3}$. The organic layer is washed with water, then dried over $\mathrm{MgSO}_{4}$ and concentrated to afford the corresponding product 1.

Yield: 98\%; 2.704 g; Beige solid; Rf: 0.32 (DCM/EtOAc, 4/1, v/v); mp 178-181 ${ }^{\circ} \mathrm{C}$; IR (neat) $v_{\max } 3062,3045,2922,2852,1658,1585,1442,1346,1284,1154,1058,983 \mathrm{~cm}^{-1} ;{ }^{1} \mathrm{H}$ NMR $\left(\mathrm{CDCl}_{3}, 25^{\circ} \mathrm{C}, 300 \mathrm{MHz}\right): \delta_{H} 9.22(1 \mathrm{H}, \mathrm{s}), 8.49(1 \mathrm{H}, \mathrm{d}, J=8.7 \mathrm{~Hz}), 8.26(1 \mathrm{H}, \mathrm{s}), 7.87(1 \mathrm{H}, \mathrm{d}, J=$ $8.8 \mathrm{~Hz}), 7.45-7.30(5 \mathrm{H}, \mathrm{m}), 5.32(2 \mathrm{H}, \mathrm{s}) ;{ }^{13} \mathrm{C} \mathrm{NMR}\left(\mathrm{CDCl}_{3}, 25{ }^{\circ} \mathrm{C}, 75.4 \mathrm{MHz}\right): \delta_{C} 160.0(\mathrm{C})$, $157.9(\mathrm{CH}), 152.7(\mathrm{C}), 147.1(\mathrm{C}), 146.2(\mathrm{CH}), 135.4(\mathrm{C}), 130.7(\mathrm{C}), 129.7(\mathrm{CH}), 129.3(2 \times \mathrm{CH})$, $128.7(\mathrm{CH}), 128.3(2 \times \mathrm{CH}), 126.3(\mathrm{CH}), 116.8(\mathrm{C}), 50.0\left(\mathrm{CH}_{2}\right)$. HRMS $\left(\mathrm{ESI}^{+}\right)$: Calcd for ${ }^{12} \mathrm{C}_{16}{ }^{1} \mathrm{H}_{12}{ }^{14} \mathrm{~N}_{3}{ }^{16} \mathrm{O}^{32} \mathrm{~S}[\mathrm{M}+\mathrm{H}]{ }^{+}:$294.0701; Found: 294.0689.

\footnotetext{
${ }^{1}$ Hédou, D.;Guillon, R.; Lecointe, C.; Logé, C.; Chosson, E.; Besson, T. Tetrahedron 2013, 69, 3182.
} 
3. General procedure for the mono-arylation<smiles>Cc1nc2ccc3nc(Cc4ccccc4)[nH]c(=O)c3c2s1</smiles>

1
1) Cul (0.5 equiv) $\mathrm{DBU}$ (2 equiv), $10 \mathrm{~min}$

2) $\mathrm{Pd}(\mathrm{OAc})_{2}(10 \mathrm{~mol} \%)$ Ar-X (2 equiv), $3-5 \mathrm{~h}$

$\operatorname{DMF}(0.4 \mathrm{M}), 120^{\circ} \mathrm{C}(\mu \mathrm{w})$<smiles>O=c1c2c(ccc3nc(Br)sc32)ncn1Cc1ccccc1</smiles>

2a-k

$N^{8}$-benzylthiazolo[5,4-f]quinazolin-9(8H)-one 1 (100 mg, $0.341 \mathrm{mmol}, 1.0$ equiv), copper iodide ( $65 \mathrm{mg}, 0.341 \mathrm{mmol}, 1$ equiv) and DBU (101 $\mu \mathrm{L}, 0.682 \mathrm{mmol}, 2.0$ equiv) in dry DMF $(850 \mu \mathrm{L})$ are added to a $2 \mathrm{~mL}$ glass microwave vial. The mixture is stirred under microwave irradiation at $120^{\circ} \mathrm{C}$ for $10 \mathrm{~min}$ ( $2 \mathrm{~min}$ ramp). Then $\mathrm{Pd}(\mathrm{OAc})_{2}$ ( $\left.7.6 \mathrm{mg}, 0.034 \mathrm{mmol}, 10 \mathrm{~mol} \%\right)$ and aryl halide $(0.682 \mathrm{mmol}, 2.0$ equiv) are added to the mixture. The reaction is then stirred under microwave irradiation at $120{ }^{\circ} \mathrm{C}$ for $3-5 \mathrm{~h}$ ( $2 \mathrm{~min}$ ramp). The resulting solution is diluted with dichloromethane, filtered through cotton and washed three times with dichloromethane. The volatiles are rotary evaporated and the crude product is purified by flash chromatography on silica gel with a gradient of dichloromethane/ethyl acetate (DCM/EtOAc, from $1 / 0$ to $4 / 1, \mathrm{v} / \mathrm{v}$ ) as eluent to afford the corresponding product, excepted for compound $\mathbf{2 i}$ (DCM/EtOAc, from $4 / 1$ to $0 / 1, v / v$ ).

$N^{8}$-benzyl-2-phenylthiazolo[5,4-f]quinazolin-9(8H)-one (2a)<smiles></smiles>

Yield: 92\%; $116 \mathrm{mg}$; Yellow powder; Rf: 0.55 (DCM/EtOAc, 4/1, v/v); mp 212-215 ${ }^{\circ} \mathrm{C}$; IR (neat) $v_{\max } 2926,1661,1584,1475,1441,1341,1154,1070 \mathrm{~cm}^{-1} ;{ }^{1} \mathrm{H} \mathrm{NMR}\left(\mathrm{CDCl}_{3}, 25^{\circ} \mathrm{C}, 300 \mathrm{MHz}\right)$ : $\delta_{H} 8.43(1 \mathrm{H}, \mathrm{d}, J=8.8 \mathrm{~Hz}), 8.25(1 \mathrm{H}, \mathrm{s}), 8.23-8.12(2 \mathrm{H}, \mathrm{m}), 7.86(1 \mathrm{H}, \mathrm{d}, J=8.7 \mathrm{~Hz}), 7.59-$ $7.47(3 \mathrm{H}, \mathrm{m}), 7.45-7.31(5 \mathrm{H}, \mathrm{m}), 5.32(2 \mathrm{H}, \mathrm{s}) ;{ }^{13} \mathrm{C} \mathrm{NMR}\left(\mathrm{CDCl}_{3}, 25^{\circ} \mathrm{C}, 75.4 \mathrm{MHz}\right): \delta_{\mathrm{C}} 171.9(\mathrm{C})$, 160.1 (C), 153.6 (C), 146.7 (C), $145.9(\mathrm{CH}), 135.5$ (C), 133.7 (C), 131.9 (C), $131.3(\mathrm{CH}), 129.3(4$ $x \mathrm{CH}), 129.2(\mathrm{CH}), 128.7(\mathrm{CH}), 128.3(2 \times \mathrm{CH}), 127.7(2 \times \mathrm{CH}), 126.3(\mathrm{CH}), 116.8(\mathrm{C}), 50.1\left(\mathrm{CH}_{2}\right)$. HRMS (ESI ${ }^{+}$): Calcd for ${ }^{12} \mathrm{C}_{22}{ }^{1} \mathrm{H}_{16}{ }^{14} \mathrm{~N}_{3}{ }^{16} \mathrm{O}^{32} \mathrm{~S}[\mathrm{M}+\mathrm{H}]^{+}$: 370.1014 ; Found: 370.1005.

$N^{8}$-benzyl-2-(p-tolyl)thiazolo[5,4-f]quinazolin-9(8H)-one (2b)<smiles>Cc1ccc(-c2nc3ccc4nc[nH]c(=O)c4c3s2)cc1</smiles> 
Yield: 86\%; $113 \mathrm{mg}$; Yellow solid; Rf: 0.61 (DCM/EtOAc, 4/1, v/v); mp 269-272 ${ }^{\circ} \mathrm{C}$; IR (neat) $v_{\max }$ 3066, 2951, 2916, 1708, 1657, 1585, 1478, 1454, 1366, 1337, 1264, 1181, 1153, 1069 $\mathrm{cm}^{-1} ;{ }^{1} \mathrm{H}$ NMR $\left(\mathrm{CDCl}_{3}, 25^{\circ} \mathrm{C}, 300 \mathrm{MHz}\right): \delta_{H} 8.41(1 \mathrm{H}, \mathrm{d}, J=8.7 \mathrm{~Hz}), 8.23(1 \mathrm{H}, \mathrm{s}), 8.15-8.00$ $(2 \mathrm{H}, \mathrm{m}), 7.84(1 \mathrm{H}, \mathrm{d}, J=8.8 \mathrm{~Hz}), 7.51-7.28(7 \mathrm{H}, \mathrm{m}), 5.32(2 \mathrm{H}, \mathrm{s}), 2.44(3 \mathrm{H}, \mathrm{s}) ;{ }^{13} \mathrm{C} \mathrm{NMR}$ $\left(\mathrm{CDCl}_{3}, 25^{\circ} \mathrm{C}, 75.4 \mathrm{MHz}\right): \delta_{\mathrm{C}} 172.1$ (C), 160.1 (C), 153.7 (C), $146.5(\mathrm{C}), 145.8(\mathrm{CH}), 141.8(\mathrm{C})$, 135.5 (C), $131.8(\mathrm{C}), 131.0$ (C), $130.0(2 \times \mathrm{CH}), 129.3(2 \times \mathrm{CH}), 129.1(\mathrm{CH}), 128.7(\mathrm{CH}), 128.3(2$ $\times \mathrm{CH}), 127.6(2 \times \mathrm{CH}), 126.2(\mathrm{CH}), 116.8(\mathrm{C}), 50.1\left(\mathrm{CH}_{2}\right), 21.7\left(\mathrm{CH}_{3}\right) . \mathrm{HRMS}\left(\mathrm{ESI}{ }^{+}\right)$: Calcd for ${ }^{12} \mathrm{C}_{23}{ }^{1} \mathrm{H}_{18}{ }^{14} \mathrm{~N}_{3}{ }^{16} \mathrm{O}^{32} \mathrm{~S}[\mathrm{M}+\mathrm{H}]{ }^{+}$: 384.1171; Found: 384.1171.

$N^{8}$-benzyl-2-(4-methoxyphenyl)thiazolo[5,4-f]quinazolin-9(8H)-one (2c)

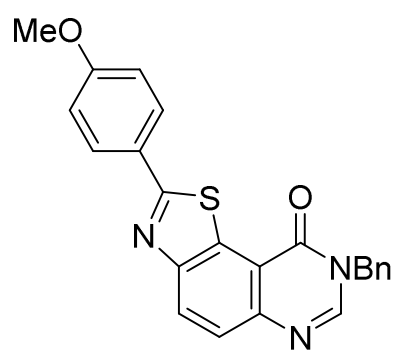

Yield: 63\%; 85 mg; Colorless solid; Rf: 0.33 (DCM/EtOAc, 4/1, v/v); mp 246-249 ${ }^{\circ} \mathrm{C}$; IR (neat) $v_{\max }$ 3067, 2938, 2838, 1657, 1600, 1478, 1452, 1403, 1337, 1262, 1169, 1152, 1069, 1032 $\mathrm{cm}^{-1} ;{ }^{1} \mathrm{H} \mathrm{NMR}\left(\mathrm{CDCl}_{3}, 25{ }^{\circ} \mathrm{C}, 300 \mathrm{MHz}\right): \delta_{H} 8.39(1 \mathrm{H}, \mathrm{d}, J=8.2 \mathrm{~Hz}), 8.23(1 \mathrm{H}, \mathrm{s}), 8.17-8.08$ $(2 \mathrm{H}, \mathrm{m}), 7.83(1 \mathrm{H}, \mathrm{d}, J=8.6 \mathrm{~Hz}), 7.46-7.29(5 \mathrm{H}, \mathrm{m}), 7.05-7.02(2 \mathrm{H}, \mathrm{m}), 5.32(2 \mathrm{H}, \mathrm{s}), 3.90$ $(3 \mathrm{H}, \mathrm{s}) ;{ }^{13} \mathrm{C} \mathrm{NMR}\left(\mathrm{CDCl}_{3}, 25{ }^{\circ} \mathrm{C}, 75.4 \mathrm{MHz}\right): \delta_{\mathrm{C}} 171.7$ (C), 162.2 (C), 160.1 (C), 153.7 (C), 146.4 (C), $145.7(\mathrm{CH}), 135.5(2 \times \mathrm{C}), 131.7(\mathrm{C}), 129.3(4 \times \mathrm{CH}), 128.8(\mathrm{CH}), 128.7(\mathrm{CH}), 128.3(2 \times \mathrm{CH})$, $\left.126.5(\mathrm{C}), 126.1(\mathrm{CH}), 114.7(2 \times \mathrm{CH}), 55.7\left(\mathrm{CH}_{3}\right), 50.1\left(\mathrm{CH}_{2}\right) . \mathrm{HRMS}(\mathrm{ESI})^{+}\right)$: Calcd for ${ }^{12} \mathrm{C}_{23}{ }^{1} \mathrm{H}_{17}{ }^{14} \mathrm{~N}_{3}{ }^{16} \mathrm{O}_{2}{ }^{32} \mathrm{~S}[\mathrm{M}+\mathrm{H}]^{+}:$: 400.1120; Found: 400.1126 .

$N^{8}$-benzyl-2-(4-chlorophenyl)thiazolo[5,4-f]quinazolin-9(8H)-one (2d)<smiles>O=c1c2c(ccc3nc(-c4ccc(Cl)cc4)sc32)ncn1Cc1ccccc1</smiles>

Yield: 59\%; $82 \mathrm{mg}$; Yellow solid; Rf: 0.51 (DCM/EtOAc, 4/1, v/v); mp 282-285 ${ }^{\circ} \mathrm{C}$; IR (neat) $v_{\max }$ 2923, 2852, 1738, 1658, 1584, 1452, 1367, 1337, 1206, 1153, $1087 \mathrm{~cm}^{-1} ;{ }^{1} \mathrm{H} \mathrm{NMR}\left(\mathrm{CDCl}_{3}, 25\right.$ $\left.{ }^{\circ} \mathrm{C}, 300 \mathrm{MHz}\right): \delta_{H} 8.42(1 \mathrm{H}, \mathrm{d}, J=8.8 \mathrm{~Hz}), 8.25(1 \mathrm{H}, \mathrm{s}), 8.13(2 \mathrm{H}, \mathrm{d}, J=8.4 \mathrm{~Hz}), 7.87(1 \mathrm{H}, \mathrm{d}, J=$ $8.8 \mathrm{~Hz}), 7.51(2 \mathrm{H}, \mathrm{d}, J=8.4 \mathrm{~Hz}), 7.45-7.31(5 \mathrm{H}, \mathrm{m}), 5.33(2 \mathrm{H}, \mathrm{s}) ;{ }^{13} \mathrm{C} \mathrm{NMR}_{(\mathrm{CDCl}}, 25{ }^{\circ} \mathrm{C}, 75.4$ $\mathrm{MHz}$ ): $\delta_{C} 170.4(\mathrm{C}), 160.0(\mathrm{C}), 153.5(\mathrm{C}), 146.8(\mathrm{CH}), 146.0(\mathrm{C}), 137.4(\mathrm{C}), 135.4(\mathrm{C}), 132.2(\mathrm{C})$, $131.9(\mathrm{C}), 129.6(2 \times \mathrm{CH}), 129.3(2 \times \mathrm{CH}), 129.3(\mathrm{CH}), 128.8(2 \times \mathrm{CH}), 128.7(\mathrm{CH}), 128.3(2 \times$ $\mathrm{CH}), 126.5(\mathrm{CH}), 115.1(\mathrm{C}), 50.1\left(\mathrm{CH}_{2}\right)$. HRMS $\left(\mathrm{ESI}^{+}\right)$: Calcd for ${ }^{12} \mathrm{C}_{22}{ }^{1} \mathrm{H}_{14}{ }^{14} \mathrm{~N}_{3}{ }^{16} \mathrm{O}^{32} \mathrm{~S}^{35} \mathrm{Cl}[\mathrm{M}+\mathrm{H}]^{+}$: 404.0624; Found: 404.0627. 
$N^{8}$-benzyl-2-(4-fluorophenyl)thiazolo[5,4-f]quinazolin-9(8H)-one (2e)<smiles></smiles>

Yield: 71\%; $94 \mathrm{mg}$; Yellow solid; Rf: 0.53 (DCM/EtOAc, 4/1, v/v); mp 234-237 ${ }^{\circ} \mathrm{C}$; IR (neat) $v_{\max }$ $3060,3032,2948,1659,1585,1478,1453,1337,1230,1153,1069,952 \mathrm{~cm}^{-1} ;{ }^{1} \mathrm{H} \mathrm{NMR}\left(\mathrm{CDCl}_{3}\right.$, $\left.25{ }^{\circ} \mathrm{C}, 300 \mathrm{MHz}\right): \delta_{H} 8.41(1 \mathrm{H}, \mathrm{d}, J=8.8 \mathrm{~Hz}), 8.24(1 \mathrm{H}, \mathrm{s}), 8.18(2 \mathrm{H}, \mathrm{dd}, J=8.8,5.4 \mathrm{~Hz}), 7.86$ $(1 \mathrm{H}, \mathrm{d}, J=8.7 \mathrm{~Hz}), 7.48-7.29(5 \mathrm{H}, \mathrm{m}), 7.22(2 \mathrm{H}, \mathrm{t}, J=8.6 \mathrm{~Hz}), 5.33(2 \mathrm{H}, \mathrm{s}) ;{ }^{13} \mathrm{C} \mathrm{NMR}\left(\mathrm{CDCl}_{3}, 25\right.$ ${ }^{\circ} \mathrm{C}, 75.4 \mathrm{MHz}$ ): $\delta_{C} 170.6(\mathrm{C}), 166.4(\mathrm{C}), 161.5$ (C, d, $\left.J=231.1 \mathrm{~Hz}\right), 160.0$ (C), 153.6 (C), 146.0 $(\mathrm{CH}), 135.4(\mathrm{C}), 132.0(\mathrm{C}), 130.0(\mathrm{C}, \mathrm{d}, J=3.0 \mathrm{~Hz}), 129.7(2 \times \mathrm{CH}, \mathrm{d}, J=8.7 \mathrm{~Hz}), 129.3(2 \times \mathrm{CH})$, $129.2(\mathrm{CH}), 128.7(\mathrm{CH}), 128.4(2 \times \mathrm{CH}), 126.4(\mathrm{CH}), 116.5(2 \times \mathrm{CH}, \mathrm{d}, J=22.2 \mathrm{~Hz}), 114.6(\mathrm{C})$, $50.1\left(\mathrm{CH}_{2}\right)$. HRMS $\left(\mathrm{ESI}^{+}\right)$: Calcd for ${ }^{12} \mathrm{C}_{22}{ }^{1} \mathrm{H}_{15}{ }^{14} \mathrm{~N}_{3}{ }^{16} \mathrm{O}^{32} \mathrm{~S}^{19} \mathrm{~F}[\mathrm{M}+\mathrm{H}]{ }^{+}$: 388.0920; Found: 388.0917.

4-( $N^{8}$-benzyl-9-oxo-8,9-dihydrothiazolo[5,4-f]quinazolin-2-yl)benzonitrile (2f)<smiles>N#Cc1ccc(-c2nc3ccc4ncn(Cc5ccccc5)c(=O)c4c3s2)cc1</smiles>

Yield: 59\%; $79 \mathrm{mg}$; Yellow solid; Rf: 0.45 (DCM/EtOAc, 4/1, v/v); $\mathrm{mp}>300{ }^{\circ} \mathrm{C}$; IR (neat) $v_{\max }$ 3057, 2950, 2226, 1739, 1655, 1583, 1451, 1338, 1152, $1068 \mathrm{~cm}^{-1} ;{ }^{1} \mathrm{H}$ NMR $\left(\mathrm{CDCl}_{3}, 25^{\circ} \mathrm{C}, 300\right.$ $\mathrm{MHz}): \delta_{H} 8.46(1 \mathrm{H}, \mathrm{d}, J=8.7 \mathrm{~Hz}), 8.33-8.27(3 \mathrm{H}, \mathrm{m}), 7.91(1 \mathrm{H}, \mathrm{d}, J=9.0 \mathrm{~Hz}), 7.82(2 \mathrm{H}, \mathrm{d}, J=$ $8.2 \mathrm{~Hz}), 7.45-7.34(5 \mathrm{H}, \mathrm{m}), 5.34(2 \mathrm{H}, \mathrm{s}) ;{ }^{13} \mathrm{C} \mathrm{NMR}\left(\mathrm{CDCl}_{3}, 25{ }^{\circ} \mathrm{C}, 75.4 \mathrm{MHz}\right): \delta_{\mathrm{C}} 169.1(\mathrm{C})$, 159.9 (C), 153.5 (C), 147.9 (C), 147.9 (C), 146.4 (CH), 137.6 (C), 135.3 (C), $133.1(2 \times \mathrm{CH})$, $129.7(\mathrm{CH}), 129.4(2 \times \mathrm{CH}), 128.8(\mathrm{CH}), 128.4(2 \times \mathrm{CH}), 128.2(\mathrm{C}), 128.1(2 \times \mathrm{CH}), 126.9(\mathrm{CH})$, 118.43 (C), 114.4 (C), $50.3\left(\mathrm{CH}_{2}\right)$. HRMS $\left(\mathrm{ESI}^{+}\right)$: Calcd for ${ }^{12} \mathrm{C}_{23}{ }^{1} \mathrm{H}_{14}{ }^{14} \mathrm{~N}_{3}{ }^{16} \mathrm{O}^{32} \mathrm{~S}[\mathrm{M}+\mathrm{H}]{ }^{+}$: 395.0967; Found: 395.0965.

$N^{8}$-benzyl-2-(2,4-dichlorophenyl)thiazolo[5,4-f]quinazolin-9(8H)-one (2g)<smiles>O=c1c2c(ccc3nc(-c4ccc(Cl)cc4Cl)sc32)ncn1Cc1ccccc1</smiles> 
Yield: 62\%; 93 mg; Yellow powder; Rf: 0.68 (DCM/EtOAc, 4/1, v/v); mp 262-265 ${ }^{\circ} \mathrm{C}$; IR (neat) $v_{\max } 3067,1664,1581,1477,1442,1348,1265,1150,1101,1063,952 \mathrm{~cm}^{-1} ;{ }^{1} \mathrm{H} \mathrm{NMR}\left(\mathrm{CDCl}_{3}\right.$, $\left.25^{\circ} \mathrm{C}, 300 \mathrm{MHz}\right): \delta_{H} 8.47(1 \mathrm{H}, \mathrm{d}, J=8.8 \mathrm{~Hz}), 8.33(1 \mathrm{H}, \mathrm{d}, J=8.6 \mathrm{~Hz}), 8.26(1 \mathrm{H}, \mathrm{s}), 7.89(1 \mathrm{H}, \mathrm{d}, J$ $=8.8 \mathrm{~Hz}), 7.59(1 \mathrm{H}, \mathrm{d}, J=2.1 \mathrm{~Hz}), 7.46-7.32(6 \mathrm{H}, \mathrm{m}), 5.34(2 \mathrm{H}, \mathrm{s}) ;{ }^{13} \mathrm{C} \mathrm{NMR}\left(\mathrm{CDCl}_{3}, 25{ }^{\circ} \mathrm{C}\right.$, $75.4 \mathrm{MHz}$ ): $\delta_{C} 167.0(\mathrm{C}), 159.9(\mathrm{C}), 151.8(\mathrm{C}), 146.7(\mathrm{C}), 146.3(\mathrm{CH}), 137.0(\mathrm{C}), 135.3(\mathrm{C}), 133.6$ (C), 133.1 (C), $132.7(\mathrm{CH}), 130.9(\mathrm{CH}), 130.8(\mathrm{C}), 129.6(\mathrm{CH}), 129.4(2 \times \mathrm{CH}), 128.8(\mathrm{CH}), 128.3$ $(2 \times \mathrm{CH}), 127.8(\mathrm{CH}), 126.2(\mathrm{CH}), 116.6(\mathrm{C}), 50.2\left(\mathrm{CH}_{2}\right)$. HRMS $\left(\mathrm{ESI}^{+}\right)$: Calcd for ${ }^{12} \mathrm{C}_{22}{ }^{1} \mathrm{H}_{14}{ }^{14} \mathrm{~N}_{3}{ }^{16} \mathrm{O}^{32} \mathrm{~S}^{35} \mathrm{Cl}_{2}[\mathrm{M}+\mathrm{H}]{ }^{+}$: 438.0235; Found: 438.0244 .

$N^{8}$-benzyl-2-(4-(dimethylamino)phenyl)thiazolo[5,4-f]quinazolin-9(8H)-one (2h)<smiles></smiles>

Yield: 87\%; $122 \mathrm{mg}$; Orange powder; Rf: 0.45 (DCM/EtOAc, 4/1, v/v); mp 224-227 ${ }^{\circ} \mathrm{C}$; IR (neat) $v_{\max } 3544,2919,2851,2803,1654,1602,1529,1444,1429,1366,1335,1263,1187$, 1150, 1067, $943 \mathrm{~cm}^{-1} ;{ }^{1} \mathrm{H}$ NMR $\left(\mathrm{CDCl}_{3}, 25{ }^{\circ} \mathrm{C}, 300 \mathrm{MHz}\right): \delta_{H} 8.32(1 \mathrm{H}, \mathrm{d}, J=8.8 \mathrm{~Hz}), 8.18(1 \mathrm{H}$, s), $8.11-7.96(2 \mathrm{H}, \mathrm{m}), 7.78(1 \mathrm{H}, \mathrm{d}, J=8.8 \mathrm{~Hz}), 7.49-7.29(5 \mathrm{H}, \mathrm{m}), 6.84-6.67(2 \mathrm{H}, \mathrm{m}), 5.29$ $(2 \mathrm{H}, \mathrm{s}), 3.06(6 \mathrm{H}, \mathrm{s}) ;{ }^{13} \mathrm{C} \mathrm{NMR}\left(\mathrm{CDCl}_{3}, 25{ }^{\circ} \mathrm{C}, 75.4 \mathrm{MHz}\right): \delta_{\mathrm{C}} 172.6$ (C), 160.2 (C), 154.0 (C), 152.4 (C), 145.9 (C), $145.3(\mathrm{CH}), 135.6$ (C), 131.3 (C), $129.2(2 \times \mathrm{CH}), 129.0(2 \times \mathrm{CH}), 128.6$ (CH), $128.3(3 \times \mathrm{CH}), 125.8(\mathrm{CH}), 121.3(\mathrm{C}), 116.6(\mathrm{C}), 111.9(2 \times \mathrm{CH}), 50.0\left(\mathrm{CH}_{2}\right), 40.3(2 \times$ $\mathrm{CH}_{3}$ ). HRMS (ESI ${ }^{+}$): Calcd for ${ }^{12} \mathrm{C}_{24}{ }^{1} \mathrm{H}_{21}{ }^{14} \mathrm{~N}_{4}{ }^{16} \mathrm{O}^{32} \mathrm{~S}[\mathrm{M}+\mathrm{H}]^{+}$: 413.1436 ; Found: 413.1431.

$N^{8}$-benzyl-2-(pyridin-3-yl)thiazolo[5,4-f]quinazolin-9(8H)-one (2i)<smiles></smiles>

Yield: 69\%; $87 \mathrm{mg}$; Brown solid; Rf: 0.08 (DCM/EtOAc, 4/1, v/v); mp 201-204 ${ }^{\circ} \mathrm{C}$; IR (neat) $v_{\max }$ 3395, 3059, 2919, 2851, 1656, 1587, 1449, 1345, 1267, 1157, 1076, $1000 \mathrm{~cm}^{-1} ;{ }^{1} \mathrm{H}$ NMR $\left(\mathrm{CDCl}_{3}, 25^{\circ} \mathrm{C}, 300 \mathrm{MHz}\right): \delta_{H} 9.41(1 \mathrm{H}, \mathrm{d}, J=2.3 \mathrm{~Hz}), 8.75(1 \mathrm{H}, \mathrm{dd}, J=4.8,1.6 \mathrm{~Hz}), 8.55-8.38$ $(2 \mathrm{H}, \mathrm{m}), 8.26(1 \mathrm{H}, \mathrm{s}), 7.89(1 \mathrm{H}, \mathrm{d}, J=8.7 \mathrm{~Hz}), 7.48(1 \mathrm{H}, \mathrm{ddd}, J=8.0,4.9,0.9 \mathrm{~Hz}), 7.45-7.33$ $(5 \mathrm{H}, \mathrm{s}), 5.33(2 \mathrm{H}, \mathrm{s}) ;{ }^{13} \mathrm{C} \mathrm{NMR}\left(\mathrm{CDCl}_{3}, 25{ }^{\circ} \mathrm{C}, 75.4 \mathrm{MHz}\right): \delta_{\mathrm{C}} 168.4(\mathrm{C}), 160.0(\mathrm{C}), 153.4(\mathrm{C})$, $151.9(\mathrm{CH}), 148.7(\mathrm{CH}), 147.0(\mathrm{C}), 146.2(\mathrm{CH}), 135.4(\mathrm{C}), 134.7(\mathrm{CH}), 131.9(\mathrm{C}), 129.8(\mathrm{C}), 129.4$ (2 x CH), $129.4(\mathrm{CH}), 128.8(\mathrm{CH}), 128.4(2 \times \mathrm{CH}), 126.7(\mathrm{CH}), 124.1(\mathrm{CH}), 116.8(\mathrm{C}), 50.2\left(\mathrm{CH}_{2}\right)$. HRMS (ESI ${ }^{+}$): Calcd for ${ }^{12} \mathrm{C}_{21}{ }^{1} \mathrm{H}_{15}{ }^{14} \mathrm{~N}_{4}{ }^{16} \mathrm{O}^{32} \mathrm{~S}[\mathrm{M}+\mathrm{H}]^{+}$: 371.0967 ; Found: 371.0957. 
$N^{8}$-benzyl-2-(pyrazin-2-yl)thiazolo[5,4-f]quinazolin-9(8H)-one (2j)<smiles>O=c1c2c(ccc3nc(-c4cnccn4)sc32)ncn1Cc1ccccc1</smiles>

Yield: 47\%; $59 \mathrm{mg}$; Brown solid; Rf: 0.16 (DCM/EtOAc, 4/1, v/v); mp 216-219 ${ }^{\circ} \mathrm{C}$; IR (neat) $v_{\max }$ $3062,3033,2953,1739,1654,1584,1449,1340,1238,1153,1068,1014 \mathrm{~cm}^{-1} ;{ }^{1} \mathrm{H}$ NMR $\left(\mathrm{CDCl}_{3}, 25^{\circ} \mathrm{C}, 300 \mathrm{MHz}\right): \delta_{H} 9.63(1 \mathrm{H}, \mathrm{s}), 8.69(2 \mathrm{H}, \mathrm{s}), 8.48(1 \mathrm{H}, \mathrm{d}, J=8.5 \mathrm{~Hz}), 8.26(1 \mathrm{H}, \mathrm{s}), 7.88$ $(1 \mathrm{H}, \mathrm{d}, J=8.8 \mathrm{~Hz}), 7.48-7.31(5 \mathrm{H}, \mathrm{m}), 5.34(2 \mathrm{H}, \mathrm{s}) ;{ }^{13} \mathrm{C} \mathrm{NMR}\left(\mathrm{CDCl}_{3}, 25{ }^{\circ} \mathrm{C}, 75.4 \mathrm{MHz}\right): \delta_{C}$ $170.0(\mathrm{C}), 159.9(\mathrm{C}), 153.7(\mathrm{C}), 147.4(\mathrm{C}), 146.4(\mathrm{CH}), 146.0(\mathrm{CH}), 144.5(\mathrm{CH}), 142.5(\mathrm{CH}), 135.4$ (C), $133.4(\mathrm{C}), 129.8(\mathrm{CH}), 129.3(2 \times \mathrm{CH}), 128.7(\mathrm{CH}), 128.4(2 \times \mathrm{CH}), 128.3(\mathrm{C}), 126.8(\mathrm{CH})$, 117.0 (C), $50.1\left(\mathrm{CH}_{2}\right)$. HRMS $\left(\mathrm{ESI}^{+}\right)$: Calcd for ${ }^{12} \mathrm{C}_{20}{ }^{1} \mathrm{H}_{13}{ }^{14} \mathrm{~N}_{5}{ }^{16} \mathrm{O}^{32} \mathrm{~S}[\mathrm{M}+\mathrm{H}]{ }^{+}:$: 372.0919; Found: 372.0909 .

(E)- $N^{8}$-benzyl-2-styrylthiazolo[5,4-f]quinazolin-9(8H)-one (2k)

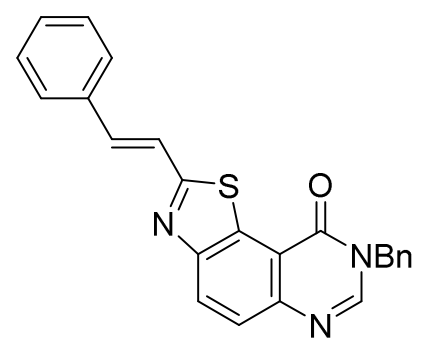

Yield: 43\%; $58 \mathrm{mg}$; Brown solid; Rf: 0.55 (DCM/EtOAc, 4/1, v/v); mp 116-119 ${ }^{\circ} \mathrm{C}$; IR (neat) $v_{\max }$ 3028, 2928, 2326, 2247, 2111, 1881, 1792, 1660, 1583, 1495, 1444, 1340, 1253, 1153, 1074, $951 \mathrm{~cm}^{-1} ;{ }^{1} \mathrm{H} \mathrm{NMR}\left(\mathrm{CDCl}_{3}, 25{ }^{\circ} \mathrm{C}, 300 \mathrm{MHz}\right): \delta_{H} 8.35(1 \mathrm{H}, \mathrm{d}, J=8.8 \mathrm{~Hz}), 8.23(1 \mathrm{H}, \mathrm{s}), 7.83(1 \mathrm{H}$, $\mathrm{d}, J=8.9 \mathrm{~Hz}), 7.69(1 \mathrm{H}, \mathrm{d}, J=16.3 \mathrm{~Hz}), 7.61(2 \mathrm{H}, \mathrm{d}, J=7.3 \mathrm{~Hz}), 7.48(2 \mathrm{H}, \mathrm{d}, J=16.2 \mathrm{~Hz}), 7.45-$ $7.32(7 \mathrm{H}, \mathrm{m}), 5.32(2 \mathrm{H}, \mathrm{s}) ;{ }^{13} \mathrm{C} \mathrm{NMR}\left(\mathrm{CDCl}_{3}, 25{ }^{\circ} \mathrm{C}, 75.4 \mathrm{MHz}\right): \delta_{\mathrm{C}} 170.9$ (C), 160.1 (C), 145.9 (CH), $138.4(\mathrm{CH}), 135.5(\mathrm{CH}), 129.8(\mathrm{CH}), 129.3(2 \times \mathrm{CH}), 129.2(3 \times \mathrm{CH}), 129.0(\mathrm{C}), 128.9(\mathrm{C})$, 128.7 (C), 128.6 (C), $128.4(2 \times \mathrm{CH}), 128.2$ (C), $127.7(2 \times \mathrm{CH}), 126.3(\mathrm{CH}), 124.1$ (C), 122.1 (CH), $50.1\left(\mathrm{CH}_{2}\right)$. HRMS (ESI $\left.{ }^{+}\right)$: Calcd for ${ }^{12} \mathrm{C}_{24}{ }^{1} \mathrm{H}_{18}{ }^{14} \mathrm{~N}_{3}{ }^{16} \mathrm{O}^{32} \mathrm{~S}[\mathrm{M}+\mathrm{H}]{ }^{+}: 396.1171$; Found: 396.1175 .

4. General procedure for the bis-arylation<smiles>Cc1nc2ccc3c(c2s1)C(=O)C(Cc1ccccc1)=N3</smiles>

1
1) Cul (0.5 equiv) $\mathrm{LiO}^{t} \mathrm{Bu}$ (2 equiv), $10 \mathrm{~min}$ 2) $\mathrm{Pd}(\mathrm{OAc})_{2}(5 \mathrm{~mol} \%)$ Ar-I (3 equiv), $4 \mathrm{~h}$

$\operatorname{DMF}(0.4 \mathrm{M}), 120^{\circ} \mathrm{C}(\mu \mathrm{w})$

$N^{8}$-benzylthiazolo[5,4-f]quinazolin-9(8H)one 1 ( $0.341 \mathrm{mmol}, 1.0$ equiv), copper iodide (33 $\mathrm{mg}, 0.17 \mathrm{mmol}, 0.5$ equiv) and lithium tert-butoxide (55 mg, $0.682 \mathrm{mmol}, 2.0$ equiv) in dry 
DMF $(850 \mu \mathrm{L})$ are added to a $2 \mathrm{~mL}$ glass microwave vial. The mixture is stirred under microwave irradiation at $120{ }^{\circ} \mathrm{C}$ for $10 \mathrm{~min}\left(2 \mathrm{~min}\right.$ ramp). Then $\mathrm{Pd}(\mathrm{OAc})_{2}(3.8 \mathrm{mg}, 0.017$ mmol, $5.0 \mathrm{~mol} \%)$ and aryl iodide $(1.023 \mathrm{mmol}, 3.0$ equiv) are added to the mixture. The reaction is then stirred under microwave irradiation at $120{ }^{\circ} \mathrm{C}$ for $4 \mathrm{~h}(2 \mathrm{~min}$ ramp). The resulting solution is diluted with dichloromethane, filtered through cotton and washed three times with dichloromethane. The volatiles are rotary evaporated and the crude product is purified by flash chromatography on silica gel with a gradient of petroleum ether /dichloromethane/ethyl acetate (PE/DCM/EtOAc, from $1 / 4 / 0$ to $0 / 1 / 0$ to $0 / 4 / 1, v / v$ ) as eluent to afford the corresponding product.

$N^{8}$-benzyl-2,7-diphenylthiazolo[5,4-f]quinazolin-9(8H)-one (3a)

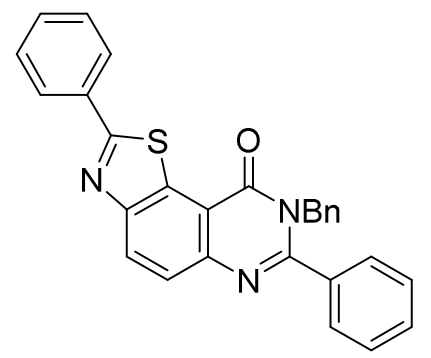

Yield: 59\%; 89 mg; Yellow solid; Rf: 0.91 (DCM/EtOAc, 4/1, v/v); mp 174-177 ${ }^{\circ} \mathrm{C}$; IR (neat) $v_{\max }$ $3057,3028,2916,2847,1685,1347,1272,1250,1158,1127,1070,1016 \mathrm{~cm}^{-1} ;{ }^{1} \mathrm{H}$ NMR $\left(\mathrm{CDCl}_{3}, 25^{\circ} \mathrm{C}, 300 \mathrm{MHz}\right): \delta_{\mathrm{H}} 8.47(1 \mathrm{H}, \mathrm{d}, J=8.8 \mathrm{~Hz}), 8.26-8.13(2 \mathrm{H}, \mathrm{m}), 7.93(1 \mathrm{H}, \mathrm{d}, J=8.7$ $\mathrm{Hz}), 7.61-7.48(4 \mathrm{H}, \mathrm{m}), 7.48-7.34(3 \mathrm{H}, \mathrm{m}), 7.25-7.14(4 \mathrm{H}, \mathrm{m}), 7.03-6.91(2 \mathrm{H} \mathrm{m}), 5.41$ $(2 \mathrm{H}, \mathrm{s}) ;{ }^{13} \mathrm{C}$ NMR $\left(\mathrm{CDCl}_{3}, 25{ }^{\circ} \mathrm{C}, 75.4 \mathrm{MHz}\right): \delta_{\mathrm{C}} 171.7$ (C), 161.5 (C), 158.8 (C), $156.1(\mathrm{C}), 153.5$ (C), 145.9 (C), 136.3 (C), 135.1 (C), 133.8 (C), 132.1 (C), $131.3(\mathrm{CH}), 130.3(\mathrm{CH}), 129.5(\mathrm{CH})$, $129.3(2 \times \mathrm{CH}), 128.9(2 \times \mathrm{CH}), 128.8(2 \times \mathrm{CH}), 128.3(2 \times \mathrm{CH}), 127.8(\mathrm{CH}), 127.7(2 \times \mathrm{CH}), 127.2$ $(2 \times \mathrm{CH}), 126.4(\mathrm{CH}), 49.3\left(\mathrm{CH}_{2}\right)$. HRMS $\left(\mathrm{ESI}^{+}\right)$: Calcd for ${ }^{12} \mathrm{C}_{28}{ }^{1} \mathrm{H}_{20}{ }^{14} \mathrm{~N}_{3}{ }^{16} \mathrm{O}^{32} \mathrm{~S}[\mathrm{M}+\mathrm{H}]{ }^{+}$: 446.1327; Found: 446.1322.

$N^{8}$-benzyl-2,7-di-p-tolylthiazolo[5,4-f]quinazolin-9(8H)-one (3b)

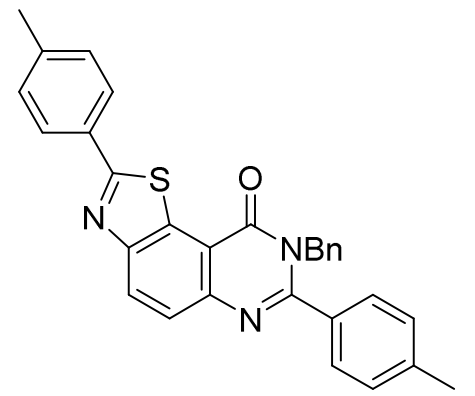

Yield: 63\%; $101 \mathrm{mg}$; Yellow solid; Rf: 0.94 (DCM/EtOAc, 4/1, v/v); mp 224-227 ${ }^{\circ} \mathrm{C}$; IR (neat) $v_{\max } 3029,2920,2853,1660,1606,1566,1452,1346,1290,1239,1153,1110,1028,958 \mathrm{~cm}^{-}$ ${ }^{1} ;{ }^{1} \mathrm{H}$ NMR $\left(\mathrm{CDCl}_{3}, 25{ }^{\circ} \mathrm{C}, 300 \mathrm{MHz}\right): \delta_{H} 8.45(1 \mathrm{H}, \mathrm{d}, J=8.7 \mathrm{~Hz}), 8.12(2 \mathrm{H}, \mathrm{d}, J=8.0 \mathrm{~Hz}), 7.91$ $(1 \mathrm{H}, \mathrm{d}, J=8.7 \mathrm{~Hz}), 7.41-7.30(5 \mathrm{H}, \mathrm{m}), 7.31-7.23(4 \mathrm{H}, \mathrm{m}), 7.07-7.00(2 \mathrm{H}, \mathrm{m}), 5.44(2 \mathrm{H}, \mathrm{s})$, $2.48(3 \mathrm{H}, \mathrm{s}), 2.46(3 \mathrm{H}, \mathrm{s}) ;{ }^{13} \mathrm{C} \mathrm{NMR}\left(\mathrm{CDCl}_{3}, 25{ }^{\circ} \mathrm{C}, 75.4 \mathrm{MHz}\right): \delta_{\mathrm{C}} 171.7(\mathrm{C}), 161.6(\mathrm{C}), 156.2(\mathrm{C})$, 153.4 (C), 145.8 (C), 141.7 (C), 140.5 (C), 136.5 (C), 132.3 (C), 131.9 (C), 131.1 (C), 130.0 (2 x $\mathrm{CH}), 129.4(2 \times \mathrm{CH}), 129.2(\mathrm{CH}), 128.7(2 \times \mathrm{CH}), 128.3(2 \times \mathrm{CH}), 127.7(\mathrm{CH}), 127.6(2 \times \mathrm{CH})$, 
$\left.127.2(2 \times \mathrm{CH}), 126.3(\mathrm{CH}), 115.4(\mathrm{C}), 49.3\left(\mathrm{CH}_{2}\right), 21.69\left(\mathrm{CH}_{3}\right), 21.59\left(\mathrm{CH}_{3}\right) . \mathrm{HRMS}(\mathrm{ESI})^{+}\right)$: Calcd for ${ }^{12} \mathrm{C}_{30}{ }^{1} \mathrm{H}_{24}{ }^{14} \mathrm{~N}_{3}{ }^{16} \mathrm{O}^{32} \mathrm{~S}[\mathrm{M}+\mathrm{H}]^{+}$: 474.1640; Found: 474.1629.

$N^{8}$-benzyl-2,7-bis(4-methoxyphenyl)thiazolo[5,4-f]quinazolin-9(8H)-one (3c)

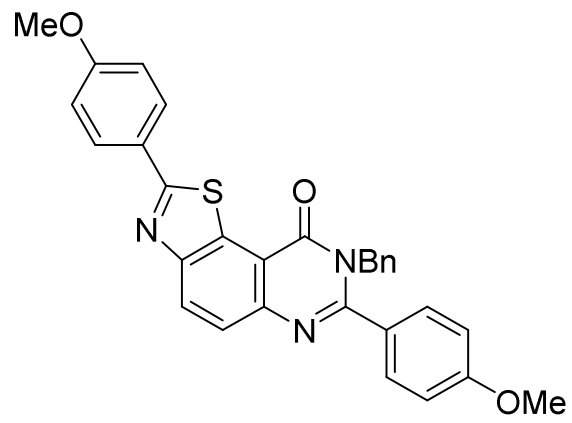

Yield: 67\%; $115 \mathrm{mg}$; Yellow solid; Rf: 0.81 (DCM/EtOAc, 4/1, v/v); mp 236-239 ${ }^{\circ} \mathrm{C}$; IR (neat) $v_{\max } 3021,2975,2942,2840,1661,1605,1578,1564,1514,1454,1421,1347,1251,1177$, $1161,1027 \mathrm{~cm}^{-1} ;{ }^{1} \mathrm{H}$ NMR $\left(\mathrm{CDCl}_{3}, 25^{\circ} \mathrm{C}, 300 \mathrm{MHz}\right): \delta_{H} 8.40(1 \mathrm{H}, \mathrm{d}, J=8.8 \mathrm{~Hz}), 8.14(2 \mathrm{H}, \mathrm{d}, J=$ $8.7 \mathrm{~Hz}), 7.87(1 \mathrm{H}, \mathrm{d}, J=8.7 \mathrm{~Hz}), 7.37(2 \mathrm{H}, \mathrm{d}, J=8.6 \mathrm{~Hz}), 7.25-7.18(3 \mathrm{H}, \mathrm{m}), 7.07-6.98(4 \mathrm{H}$, $\mathrm{m}), 6.94(2 \mathrm{H}, \mathrm{d}, J=8.6 \mathrm{~Hz}), 5.42(2 \mathrm{H}, \mathrm{s}), 3.90(3 \mathrm{H}, \mathrm{s}), 3.86(3 \mathrm{H}, \mathrm{s}) ;{ }^{13} \mathrm{C} \mathrm{NMR}\left(\mathrm{CDCl}_{3}, 25{ }^{\circ} \mathrm{C}, 75.4\right.$ $\mathrm{MHz}$ ): $\delta_{C} 171.4(\mathrm{C}), 162.2$ (C), 161.7 (C), 161.1 (C), 155.9 (C), 153.5 (C), 145.7 (C), 136.6 (C), $131.9(\mathrm{C}), 130.0(2 \times \mathrm{CH}), 129.3(2 \times \mathrm{CH}), 129.1(\mathrm{CH}), 128.8(2 \times \mathrm{CH}), 127.7(\mathrm{CH}), 127.6(\mathrm{C})$, $127.1(2 \times \mathrm{CH}), 126.6(\mathrm{C}), 126.2(\mathrm{CH}), 115.3(\mathrm{C}), 114.7(2 \times \mathrm{CH}), 114.2(2 \times \mathrm{CH}), 55.6\left(\mathrm{CH}_{3}\right), 55.6$ $\left(\mathrm{CH}_{3}\right), 49.4\left(\mathrm{CH}_{2}\right)$. HRMS $\left(\mathrm{ESI}^{+}\right)$: Calcd for ${ }^{12} \mathrm{C}_{30}{ }^{1} \mathrm{H}_{24}{ }^{14} \mathrm{~N}_{3}{ }^{16} \mathrm{O}_{3}{ }^{32} \mathrm{~S}[\mathrm{M}+\mathrm{H}]{ }^{+}$: 506.1538; Found: 506.1530 .

$N^{8}$-benzyl-2,7-bis(4-chlorophenyl)thiazolo[5,4-f]quinazolin-9(8H)-one (3d)

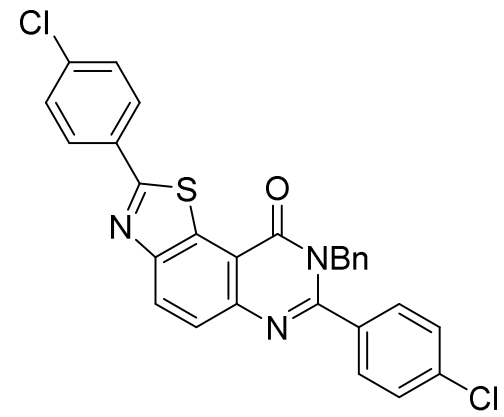

Yield: 56\%; $98 \mathrm{mg}$; Yellow solid; Rf: 0.98 (DCM/EtOAc, 4/1, v/v); mp 204-207 ${ }^{\circ} \mathrm{C}$; IR (neat) $v_{\max }$ 3048, 2951, 2361, 2118, 1899, 1664, 1574, 1491, 1471, 1344, 1290, 1239, 1152, 1089, 1013, $958 \mathrm{~cm}^{-1} ;{ }^{1} \mathrm{H} \mathrm{NMR}\left(\mathrm{CDCl}_{3}, 25^{\circ} \mathrm{C}, 300 \mathrm{MHz}\right): \delta_{H} 8.46(1 \mathrm{H}, \mathrm{d}, J=8.8 \mathrm{~Hz}), 8.14(2 \mathrm{H}, \mathrm{d}, J=8.4 \mathrm{~Hz})$, $7.91(1 \mathrm{H}, \mathrm{d}, J=8.8 \mathrm{~Hz}), 7.51(2 \mathrm{H}, \mathrm{d}, J=8.6 \mathrm{~Hz}), 7.42(2 \mathrm{H}, \mathrm{d}, J=8.4 \mathrm{~Hz}), 7.34(2 \mathrm{H}, \mathrm{d}, J=8.4 \mathrm{~Hz})$, $7.26-7.22(3 \mathrm{H}, \mathrm{m}), 7.06-6.87(2 \mathrm{H}, \mathrm{m}), 5.39(2 \mathrm{H}, \mathrm{s}) ;{ }^{13} \mathrm{C} \mathrm{NMR}\left(\mathrm{CDCl}_{3}, 25{ }^{\circ} \mathrm{C}, 75.4 \mathrm{MHz}\right): \delta_{\mathrm{C}}$ 170.4 (C), 161.4 (C), 155.2 (C), 153.5 (C), 145.9 (C), 137.4 (C), 136.6 (2 x C), 136.1 (C), 133.5 (C), $132.2(\mathrm{C}), 132.2(\mathrm{C}), 129.8(2 \times \mathrm{CH}), 129.6(3 \times \mathrm{CH}), 129.1(2 \times \mathrm{CH}), 128.9(2 \times \mathrm{CH}), 128.9(2$ $x \mathrm{CH}), 128.0(\mathrm{CH}), 127.0(2 \times \mathrm{CH}), 126.6(\mathrm{CH}), 49.33\left(\mathrm{CH}_{2}\right)$. HRMS $\left(\mathrm{ESI}^{+}\right)$: Calcd for ${ }^{12} \mathrm{C}_{28}{ }^{1} \mathrm{H}_{18}{ }^{14} \mathrm{~N}_{3}{ }^{16} \mathrm{O}^{32} \mathrm{~S}^{35} \mathrm{Cl}_{2}[\mathrm{M}+\mathrm{H}]{ }^{+}:$514.0548; Found: 514.0532. 
$N^{8}$-benzyl-2,7-bis(4-fluorophenyl)thiazolo[5,4-f]quinazolin-9(8H)-one (3e)

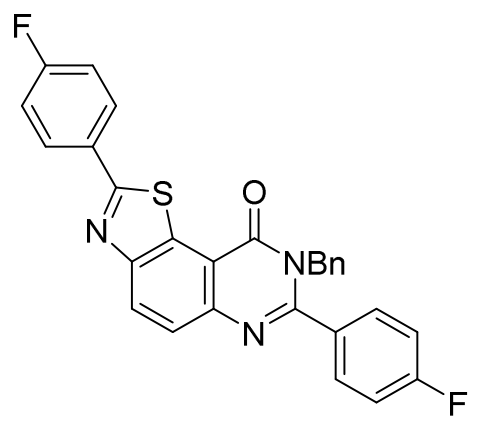

Yield: 26\%; $42 \mathrm{mg}$; Yellow solid; Rf: 0.80 (DCM/EtOAc, 4/1, v/v); mp 205-208 ${ }^{\circ} \mathrm{C}$; IR (neat) $v_{\max }$ 3057, 3043, 2999, 1667, 1574, 1511, 1458, 1343, 1229, $1156 \mathrm{~cm}^{-1} ;{ }^{1} \mathrm{H}$ NMR $\left(\mathrm{CDCl}_{3}, 25^{\circ} \mathrm{C}, 300\right.$ $\mathrm{MHz}): \delta_{H} 8.43(1 \mathrm{H}, \mathrm{d}, J=8.8 \mathrm{~Hz}), 8.22-8.15(2 \mathrm{H}, \mathrm{m}), 7.87(1 \mathrm{H}, \mathrm{d}, J=8.8 \mathrm{~Hz}), 7.43-7.34(2 \mathrm{H}$, m), $7.26-7.17(5 \mathrm{H}, \mathrm{m}), 7.16-7.08(2 \mathrm{H}, \mathrm{m}), 6.99-6.93(2 \mathrm{H}, \mathrm{m}), 5.38(2 \mathrm{H}, \mathrm{s}) ;{ }^{13} \mathrm{C}\left\{{ }^{1} \mathrm{H}\right\} \mathrm{NMR}$ $\left(\mathrm{CDCl}_{3}, 25^{\circ} \mathrm{C}, 75.4 \mathrm{MHz}\right): \delta_{\mathrm{C}} 170.5(\mathrm{C}), 164.7(\mathrm{C}, \mathrm{d}, J=252.2 \mathrm{~Hz}), 163.7(\mathrm{C}, \mathrm{d}, J=251.2 \mathrm{~Hz}$ ), 161.5 (C), 155.2 (C), 153.5 (C), 145.7 (C), 136.2 (C), 132.1 (C), 131.2 (C, d, J = 3.6 Hz), 130.5 (2 $x \mathrm{CH}, \mathrm{d}, J=8.6 \mathrm{~Hz}), 130.1(\mathrm{C}, \mathrm{d}, J=3.3 \mathrm{~Hz}), 129.7(2 \times \mathrm{CH}, \mathrm{d}, J=8.7 \mathrm{~Hz}), 129.5(\mathrm{CH}), 128.9(2 \mathrm{x}$ $\mathrm{CH}), 127.9(\mathrm{CH}), 127.0(2 \times \mathrm{CH}), 126.5(\mathrm{CH}), 116.5(2 \times \mathrm{CH}, \mathrm{d}, J=22.1 \mathrm{~Hz}), 116.0(2 \times \mathrm{CH}, \mathrm{d}, J=$ $22.0 \mathrm{~Hz}), 115.4(\mathrm{C}), 49.3\left(\mathrm{CH}_{2}\right)$. HRMS $\left(\mathrm{ESI}^{+}\right)$: Calcd for ${ }^{12} \mathrm{C}_{28}{ }^{1} \mathrm{H}_{17}{ }^{14} \mathrm{~N}_{3}{ }^{16} \mathrm{O}^{32} \mathrm{~S}^{19} \mathrm{~F}_{2}[\mathrm{M}+\mathrm{H}]^{+}$: 482.1139; Found: 482.1132.

4,4'-(N-benzyl-9-oxo-8,9-dihydrothiazolo[5,4-f]quinazoline-2,7-diyl)dibenzonitrile (3f)

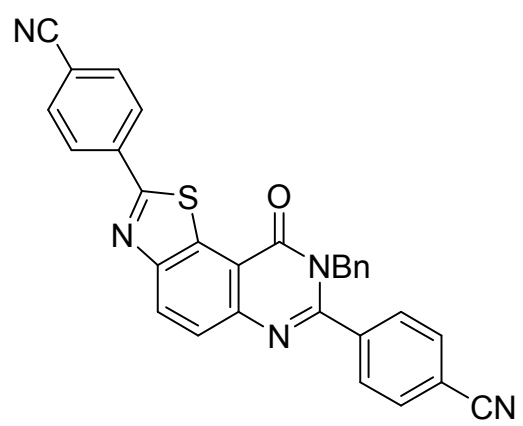

Yield: 55\%; $93 \mathrm{mg}$; Yellow solid; Rf: 0.84 (DCM/EtOAc, 4/1, v/v); $\mathrm{mp}>300{ }^{\circ} \mathrm{C}$; IR (neat) $v_{\max }$ $3051,2922,2852,2230,1658,1574,1554,1353,1291,1237,1149,1061,1016 \mathrm{~cm}^{-1} ;{ }^{1} \mathrm{H}$ NMR $\left(\mathrm{CDCl}_{3}, 25^{\circ} \mathrm{C}, 300 \mathrm{MHz}\right): \delta_{H} 8.51(1 \mathrm{H}, \mathrm{d}, J=8.8 \mathrm{~Hz}), 8.40-8.22(2 \mathrm{H}, \mathrm{m}), 7.92(1 \mathrm{H}, \mathrm{d}, J=8.8$ $\mathrm{Hz}), 7.89-7.78(2 \mathrm{H}, \mathrm{m}), 7.76-7.68(2 \mathrm{H}, \mathrm{m}), 7.53-7.45(2 \mathrm{H}, \mathrm{m}), 7.26-7.15(3 \mathrm{H}, \mathrm{m}), 6.99-$ $6.84(2 \mathrm{H}, \mathrm{m}), 5.37(2 \mathrm{H}, \mathrm{s}) ;{ }^{13} \mathrm{C}\left\{{ }^{1} \mathrm{H}\right\} \mathrm{NMR}\left(\mathrm{CDCl}, 25^{\circ} \mathrm{C}, 75.4 \mathrm{MHz}\right): \delta_{\mathrm{C}} 169.4(\mathrm{C}), 161.1(\mathrm{C}), 154.6$ (C), 153.7 (C), 146.1 (C), 139.0 (C), 137.5 (C), 135.7 (C), $133.1(2 \times \mathrm{CH}), 132.6$ (C), $132.6(2 \times$ $\mathrm{CH}), 130.2(\mathrm{CH}), 129.2(2 \times \mathrm{CH}), 129.1(2 \times \mathrm{CH}), 128.2(\mathrm{CH}), 128.1(2 \times \mathrm{CH}), 127.0(\mathrm{CH}), 126.9$ $(2 \times \mathrm{CH}), 118.4$ (C), 118.0 (C), 115.6 (C), 114.5 (C), 114.3 (C), $49.23\left(\mathrm{CH}_{2}\right)$. HRMS $\left(\mathrm{ESI}^{+}\right)$: Calcd for ${ }^{12} \mathrm{C}_{30}{ }^{1} \mathrm{H}_{18}{ }^{14} \mathrm{~N}_{5}{ }^{16} \mathrm{O}^{32} \mathrm{~S}[\mathrm{M}+\mathrm{H}]^{+}$: 496.1232; Found: 496.1237. 
5. General procedure for the di-arylation<smiles></smiles>

2
1) Cul ( 0.5 equiv) $\mathrm{LiO}^{t} \mathrm{Bu}$ (2 equiv), 10 min

2) $\mathrm{Pd}(\mathrm{OAc})_{2}(5 \mathrm{~mol} \%)$ $\mathrm{Ar}^{2}-\mathrm{I}(2$ equiv), $5 \mathrm{~h}$

$\operatorname{DMF}(0.4 \mathrm{M}), 120^{\circ} \mathrm{C}(\mu \mathrm{w})$<smiles></smiles>

4a-f

2-aryl- $N^{8}$-benzylthiazolo[5,4-f]quinazolin-9(8H)-one 2 ( $0.271 \mathrm{mmol}, 1.0$ equiv), copper iodide ( $0.136 \mathrm{mmol}, 0.5$ equiv) and lithium tert-butoxide $(0.542 \mathrm{mmol}, 2.0$ equiv) in dry DMF (0.4 $\mathrm{M})$ are added to a $2 \mathrm{~mL}$ glass microwave vial. The mixture is stirred under microwave irradiation at $120^{\circ} \mathrm{C}$ for $10 \mathrm{~min}\left(2 \mathrm{~min}\right.$ ramp). Then $\mathrm{Pd}(\mathrm{OAc})_{2}(0.014 \mathrm{mmol}, 5 \mathrm{~mol} \%)$ and aryl iodide ( $0.542 \mathrm{mmol}, 2.0$ equiv) are added to the mixture. The reaction is then stirred under microwave irradiation at $120{ }^{\circ} \mathrm{C}$ for $5 \mathrm{~h}$ ( $2 \mathrm{~min}$ ramp). The resulting solution is diluted with dichloromethane, filtered through cotton and washed three times with dichloromethane. The volatiles are rotary evaporated and the crude product is purified by flash chromatography on silica gel with a gradient of petroleum ether /dichloromethane/ethyl acetate (PE/DCM/EtOAc, from $1 / 4 / 0$ to $0 / 1 / 0$ to $0 / 4 / 1, v / v$ ) as eluent to afford the corresponding product.

$N^{8}$-benzyl-2-phenyl-7-(p-tolyl)thiazolo[5,4-f]quinazolin-9(8H)-one (4a)

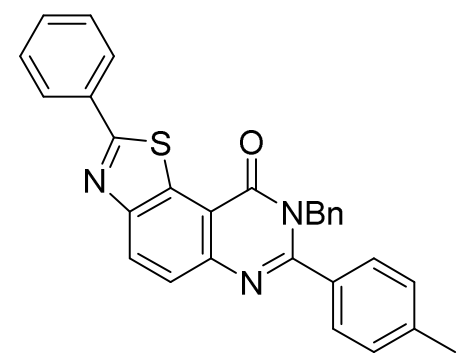

Yield: 87\%; $108 \mathrm{mg}$; Yellow solid; Rf: 0.98 (DCM/EtOAc, 4/1, v/v); mp 234-237 ${ }^{\circ} \mathrm{C}$; IR (neat) $v_{\max } 3067,3021,2922,1666,1577,1564,1545,1454,1349,1251,1160,968 \mathrm{~cm}^{-1} ;{ }^{1} \mathrm{H}$ NMR $\left(\mathrm{CDCl}_{3}, 25^{\circ} \mathrm{C}, 300 \mathrm{MHz}\right): \delta_{H} 8.45(1 \mathrm{H}, \mathrm{d}, J=8.8 \mathrm{~Hz}), 8.27-8.11(2 \mathrm{H}, \mathrm{m}), 7.91(1 \mathrm{H}, \mathrm{d}, J=8.8$ $\mathrm{Hz}), 7.56-7.49(3 \mathrm{H}, \mathrm{m}), 7.36-7.28(2 \mathrm{H}, \mathrm{m}), 7.26-7.17(5 \mathrm{H}, \mathrm{m}), 7.03-6.97(2 \mathrm{H}, \mathrm{m}), 5.41$ $(2 \mathrm{H}, \mathrm{s}), 2.43(3 \mathrm{H}, \mathrm{s}) ;{ }^{13} \mathrm{C}\left\{{ }^{1} \mathrm{H}\right\} \mathrm{NMR}\left(\mathrm{CDCl}_{3}, 25{ }^{\circ} \mathrm{C}, 75.4 \mathrm{MHz}\right): \delta_{\mathrm{C}} 171.7(\mathrm{C}), 161.5(\mathrm{C}), 156.4(\mathrm{C})$, 153.4 (C), 145.8 (C), 140.6 (C), 136.4 (2 x C), 133.8 (C), 132.1 (C), $131.3(\mathrm{CH}), 129.5(2 \times \mathrm{CH})$, $129.5(\mathrm{CH}), 129.3(2 \times \mathrm{CH}), 128.8(2 \times \mathrm{CH}), 128.3(2 \times \mathrm{CH}), 127.8(\mathrm{CH}), 127.7(2 \times \mathrm{CH}), 127.2(2$ x CH), $\left.126.3(\mathrm{CH}), 115.4(\mathrm{C}), 49.4\left(\mathrm{CH}_{2}\right), 21.6\left(\mathrm{CH}_{3}\right) . \mathrm{HRMS}(\mathrm{ESI})^{+}\right)$: Calcd for ${ }^{12} \mathrm{C}_{29}{ }^{1} \mathrm{H}_{22}{ }^{14} \mathrm{~N}_{3}{ }^{16} \mathrm{O}^{32} \mathrm{~S}$ $[\mathrm{M}+\mathrm{H}]^{+}:$460.1484; Found: 460.1506. 
4-( $N^{8}$-benzyl-9-oxo-2-(p-tolyl)-8,9-dihydrothiazolo[5,4-f]quinazolin-7-yl)benzonitrile (4b)

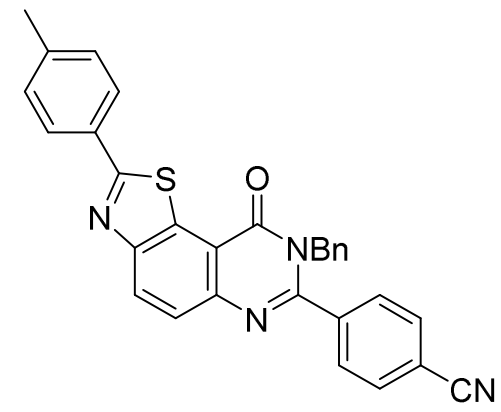

Yield: 41\%; 54 mg; Yellow solid; Rf: 0.67 (DCM/EtOAc, 4/1, v/v); mp 256-259 ${ }^{\circ} \mathrm{C}$; IR (neat) $v_{\max }$ $3020,2970,2930,2227,1739,1662,1575,1453,1348,1230,1156,1071,1013 \mathrm{~cm}^{-1} ;{ }^{1} \mathrm{H}$ NMR $\left(\mathrm{CDCl}_{3}, 25^{\circ} \mathrm{C}, 300 \mathrm{MHz}\right): \delta_{H} 8.43(1 \mathrm{H}, \mathrm{d}, J=8.7 \mathrm{~Hz}), 8.08(2 \mathrm{H}, \mathrm{d}, J=7.9 \mathrm{~Hz}), 7.84(1 \mathrm{H}, \mathrm{d}, J=8.7$ $\mathrm{Hz}), 7.70(2 \mathrm{H}, \mathrm{d}, J=8.0 \mathrm{~Hz}), 7.48(2 \mathrm{H}, \mathrm{d}, J=8.0 \mathrm{~Hz}), 7.33(2 \mathrm{H}, \mathrm{d}, J=7.9 \mathrm{~Hz}), 7.29-7.19(3 \mathrm{H}$, $\mathrm{m}), 6.95-6.85(2 \mathrm{H}, \mathrm{m}), 5.33(2 \mathrm{H}, \mathrm{s}), 2.44(3 \mathrm{H}, \mathrm{s}) ;{ }^{13} \mathrm{C}\left\{{ }^{1} \mathrm{H}\right\} \mathrm{NMR}\left(\mathrm{CDCl}_{3}, 25{ }^{\circ} \mathrm{C}, 75.4 \mathrm{MHz}\right): \delta_{\mathrm{C}}$ 172.3 (C), 161.2 (C), 153.9 (C), 145.3 (C), 142.0 (2 x C), 139.2 (C), 135.9 (C), 132.5 (2 x CH), 132.1 (C), 130.9 (C), $130.0(2 \times \mathrm{CH}), 129.5(\mathrm{CH}), 129.2(2 \times \mathrm{CH}), 129.0(2 \times \mathrm{CH}), 128.1(\mathrm{CH})$, $127.6(2 \times \mathrm{CH}), 126.9(2 \times \mathrm{CH}), 126.3(\mathrm{CH}), 118.0(\mathrm{C}), 115.5(\mathrm{C}), 114.1(\mathrm{C}), 49.1\left(\mathrm{CH}_{2}\right), 21.7$ $\left(\mathrm{CH}_{3}\right)$. HRMS $\left(\mathrm{ESI}^{+}\right)$: Calcd for ${ }^{12} \mathrm{C}_{30}{ }^{1} \mathrm{H}_{20}{ }^{14} \mathrm{~N}_{4}{ }^{16} \mathrm{O}^{32} \mathrm{~S}[\mathrm{M}+\mathrm{H}]^{+}:$: 485.1436 ; Found: 485.1427.

4-( $N^{8}$-benzyl-2-(4-methoxyphenyl)-9-oxo-8,9-dihydrothiazolo[5,4-f]quinazolin-7-yl)benzonitrile (4ca)

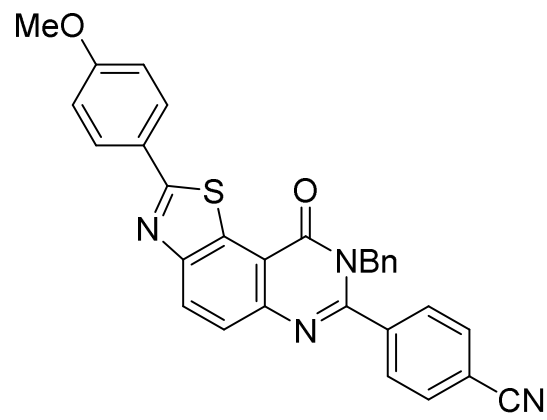

Yield: 73\%; $99 \mathrm{mg}$; Yellow solid; Rf: 0.79 (DCM/EtOAc, 4/1, v/v); mp 226-229 ${ }^{\circ} \mathrm{C}$; IR (neat) $v_{\max }$ 3064, 2937, 2839, 2231, 1672, 1605, 1573, 1477, 1452, 1415, 1344, 1311, 1258, 1159, 1116, 1031, $969 \mathrm{~cm}^{-1} ;{ }^{1} \mathrm{H}$ NMR $\left(\mathrm{CDCl}_{3}, 25^{\circ} \mathrm{C}, 300 \mathrm{MHz}\right): \delta_{H} 8.42(1 \mathrm{H}, \mathrm{d}, J=8.8 \mathrm{~Hz}), 8.13(2 \mathrm{H}, \mathrm{d}, J=$ $8.8 \mathrm{~Hz}), 7.83(1 \mathrm{H}, \mathrm{d}, J=8.8 \mathrm{~Hz}), 7.70(2 \mathrm{H}, \mathrm{d}, J=8.1 \mathrm{~Hz}), 7.48(2 \mathrm{H}, \mathrm{d}, J=8.1 \mathrm{~Hz}), 7.26-7.20$ $(3 \mathrm{H}, \mathrm{m}), 7.03(2 \mathrm{H}, \mathrm{d}, J=8.7 \mathrm{~Hz}), 6.97-6.82(2 \mathrm{H}, \mathrm{m}), 5.34(2 \mathrm{H}, \mathrm{s}), 3.90(3 \mathrm{H}, \mathrm{s}) ;{ }^{13} \mathrm{C}\left\{{ }^{1} \mathrm{H}\right\} \mathrm{NMR}$ $\left(\mathrm{CDCl}_{3}, 25{ }^{\circ} \mathrm{C}, 75.4 \mathrm{MHz}\right): \delta_{C} 172.0$ (C), 162.3 (C), $161.2(\mathrm{C}), 153.9$ (C), 153.8 (C), $145.2(\mathrm{C})$, 139.2 (C), 135.9 (C), $132.5(2 \times \mathrm{CH}), 132.0(\mathrm{C}), 129.3(3 \times \mathrm{CH}), 129.2(2 \times \mathrm{CH}), 129.0(2 \times \mathrm{CH})$, $128.1(\mathrm{CH}), 126.9(2 \times \mathrm{CH}), 126.4(\mathrm{C}), 126.2(\mathrm{CH}), 118.1(\mathrm{C}), 115.5(\mathrm{C}), 114.7(2 \times \mathrm{CH}), 114.1$ (C), $55.6\left(\mathrm{CH}_{2}\right), 49.1\left(\mathrm{CH}_{3}\right)$. HRMS (ESI $\left.{ }^{+}\right)$: Calcd for ${ }^{12} \mathrm{C}_{30}{ }^{1} \mathrm{H}_{21}{ }^{14} \mathrm{~N}_{4}{ }^{16} \mathrm{O}_{2}{ }^{32} \mathrm{~S}[\mathrm{M}+\mathrm{H}]{ }^{+}: 501.1385$; Found: 501.1379. 
$N^{8}$-benzyl-7-(4-fluorophenyl)-2-(4-methoxyphenyl)thiazolo[5,4-f]quinazolin-9(8H)-one (4cb)

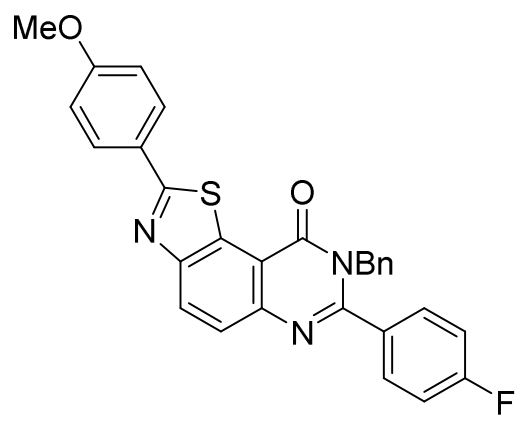

Yield: 77\%; $103 \mathrm{mg}$; Yellow solid; Rf: 0.66 (DCM/EtOAc, 4/1, v/v); mp 244-247 ${ }^{\circ} \mathrm{C}$; IR (neat) $v_{\max } 3074,3030,2957,2839,1667,1604,1569,1496,1453,1343,1246,1224,1172,1151$, $1023 \mathrm{~cm}^{-1} ;{ }^{1} \mathrm{H} \mathrm{NMR}\left(\mathrm{CDCl}_{3}, 25^{\circ} \mathrm{C}, 300 \mathrm{MHz}\right): \delta_{H} 8.40(1 \mathrm{H}, \mathrm{d}, J=8.7 \mathrm{~Hz}), 8.13(2 \mathrm{H}, \mathrm{d}, J=8.6$ $\mathrm{Hz}), 7.84(1 \mathrm{H}, \mathrm{d}, J=8.7 \mathrm{~Hz}), 7.38(2 \mathrm{H}, \mathrm{dd}, J=8.5,5.2 \mathrm{~Hz}), 7.27-7.20(3 \mathrm{H}, \mathrm{m}), 7.11(2 \mathrm{H}, \mathrm{t}, J=$ $8.5 \mathrm{~Hz}), 7.03(2 \mathrm{H}, \mathrm{d}, J=8.6 \mathrm{~Hz}), 7.01-6.91(2 \mathrm{H}, \mathrm{m}), 5.37(2 \mathrm{H}, \mathrm{s}), 3.89(3 \mathrm{H}, \mathrm{s}) ;{ }^{13} \mathrm{C}\left\{{ }^{1} \mathrm{H}\right\} \mathrm{NMR}$ $\left(\mathrm{CDCl}_{3}, 25^{\circ} \mathrm{C}, 75.4 \mathrm{MHz}\right): \delta_{\mathrm{C}} 171.6(\mathrm{C}), 163.7(\mathrm{C}, \mathrm{d}, J=250.9 \mathrm{~Hz}), 162.2$ (C), 161.6 (C), 154.9 (C), 153.7 (C), 145.4 (C), 136.3 (C), 131.9 (C), 131.3 (C, d, J = 3.6 Hz), 130.5 (2 x CH, d, J = 8.6 $\mathrm{Hz}), 129.3(2 \times \mathrm{CH}), 129.2(\mathrm{CH}), 128.9(2 \times \mathrm{CH}), 127.9(\mathrm{CH}), 127.0(2 \times \mathrm{CH}), 126.6(\mathrm{C}), 126.2$ $(\mathrm{CH}), 116.0(2 \times \mathrm{CH}, \mathrm{d}, J=21.9 \mathrm{~Hz}), 115.4(\mathrm{C}), 114.7(2 \times \mathrm{CH}), 55.7\left(\mathrm{CH}_{3}\right), 49.3\left(\mathrm{CH}_{2}\right)$. HRMS (ESI ${ }^{+}$): Calcd for ${ }^{12} \mathrm{C}_{29}{ }^{1} \mathrm{H}_{20}{ }^{14} \mathrm{~N}_{3}{ }^{16} \mathrm{O}_{2}{ }^{32} \mathrm{~S}^{19} \mathrm{~F}[\mathrm{M}+\mathrm{H}]^{+}:$494.1327; Found: 494.1336.

4-( $N^{8}$-benzyl-2-(4-chlorophenyl)-9-oxo-8,9-dihydrothiazolo[5,4-f]quinazolin-7-yl)benzonitrile (4da)

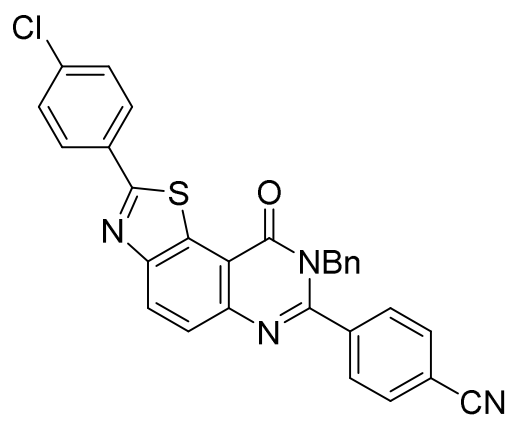

Yield: 58\%; $79 \mathrm{mg}$; Yellow solid; Rf: 0.92 (DCM/EtOAc, 4/1, v/v); mp 262-265 ${ }^{\circ} \mathrm{C}$; IR (neat) $v_{\max }$ 2932, 2922, 2230, 1739, 1665, 1575, 1441, 1342, 1234, 1152, 1089, 1063, 1014, 956, $831 \mathrm{~cm}$ 1; ${ }^{1} \mathrm{H}$ NMR $\left(\mathrm{CDCl}_{3}, 25{ }^{\circ} \mathrm{C}, 300 \mathrm{MHz}\right): \delta_{H} 8.47(1 \mathrm{H}, \mathrm{d}, J=8.8 \mathrm{~Hz}), 8.15(2 \mathrm{H}, \mathrm{d}, J=8.3 \mathrm{~Hz}), 7.88$ $(1 \mathrm{H}, \mathrm{d}, J=8.7 \mathrm{~Hz}), 7.72(2 \mathrm{H}, \mathrm{d}, J=8.0 \mathrm{~Hz}), 7.55-7.50(4 \mathrm{H}, \mathrm{m}), 7.26-7.16(3 \mathrm{H}, \mathrm{m}), 6.97-$ $6.86(2 \mathrm{H}, \mathrm{m}), 5.36(2 \mathrm{H}, \mathrm{s}) ;{ }^{13} \mathrm{C}\left\{{ }^{1} \mathrm{H}\right\} \mathrm{NMR}\left(\mathrm{CDCl}_{3}, 25^{\circ} \mathrm{C}, 75.4 \mathrm{MHz}\right): \delta_{\mathrm{C}} 174.7(\mathrm{C}), 161.7(\mathrm{C}), 161.2$ (C), 154.2 (C), 153.8 (C), 145.7 (C), 139.1 (C), 137.6 (C), 135.8 (C), 132.5 (2 x CH), 132.2 (C), 129.8 (C), $129.7(2 \times \mathrm{CH}), 129.6(\mathrm{CH}), 129.2(2 \times \mathrm{CH}), 129.1(2 \times \mathrm{CH}), 128.9(2 \times \mathrm{CH}), 128.2$ (CH), $126.9(2 \times \mathrm{CH}), 126.6(\mathrm{CH}), 118.0(\mathrm{C}), 114.2(\mathrm{C}), 49.2\left(\mathrm{CH}_{2}\right)$. HRMS (ESI $\left.{ }^{+}\right)$: Calcd for ${ }^{12} \mathrm{C}_{29}{ }^{1} \mathrm{H}_{18}{ }^{14} \mathrm{~N}_{4}{ }^{16} \mathrm{O}^{32} \mathrm{~S}^{35} \mathrm{Cl}[\mathrm{M}+\mathrm{H}]^{+}$: 505.0890; Found: 505.0886. 
$N^{8}$-benzyl-2-(4-chlorophenyl)-7-( $p$-tolyl)thiazolo[5,4-f]quinazolin-9(8H)-one (4db)

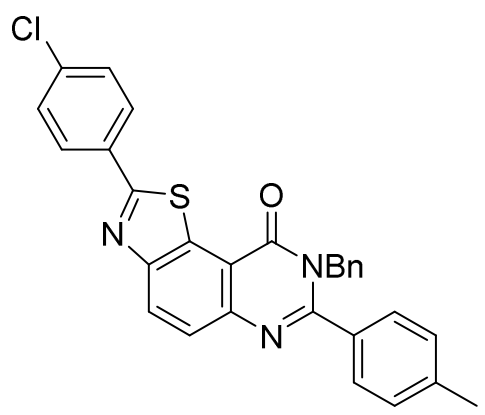

Yield: 35\%; 47 mg; White solid; Rf: 0.92 (DCM/EtOAc, 4/1, v/v); mp 228-231 ${ }^{\circ} \mathrm{C}$; IR (neat) $v_{\max }$ 3054, 3029, 1737, 1561, 1541, 1451, 1349, 1297, 1247, 1159, 1090, $1017 \mathrm{~cm}^{-1} ;{ }^{1} \mathrm{H}$ NMR $\left(\mathrm{CDCl}_{3}, 25^{\circ} \mathrm{C}, 300 \mathrm{MHz}\right): \delta_{H} 8.36(1 \mathrm{H}, \mathrm{d}, J=8.8 \mathrm{~Hz}), 8.06(2 \mathrm{H}, \mathrm{d}, J=8.5 \mathrm{~Hz}), 7.84(1 \mathrm{H}, \mathrm{d}, J=8.7$ $\mathrm{Hz}), 7.43(2 \mathrm{H}, \mathrm{d}, J=8.5 \mathrm{~Hz}), 7.25(2 \mathrm{H}, \mathrm{d}, J=7.9 \mathrm{~Hz}), 7.20-7.13(5 \mathrm{H}, \mathrm{m}), 6.98-6.86(2 \mathrm{H}, \mathrm{m})$, $5.34(2 \mathrm{H}, \mathrm{s}), 2.36(3 \mathrm{H}, \mathrm{s}) ;{ }^{13} \mathrm{C}\left\{{ }^{1} \mathrm{H}\right\} \mathrm{NMR}\left(\mathrm{CDCl}_{3}, 25^{\circ} \mathrm{C}, 75.4 \mathrm{MHz}\right): \delta_{\mathrm{C}} 170.2(\mathrm{C}), 161.5(\mathrm{C}), 156.5$ (C), 153.3 (C), 146.0 (C), 140.6 (C), 137.3 (C), 136.4 (C), 132.3 (C), 132.2 (C), 132.1 (C), 129.6 $(2 \times \mathrm{CH}), 129.5(2 \times \mathrm{CH}), 129.4(\mathrm{CH}), 128.8(2 \times \mathrm{CH}), 128.8(2 \times \mathrm{CH}), 128.3(2 \times \mathrm{CH}), 127.8(\mathrm{CH})$, $127.2(2 \times \mathrm{CH}), 126.6(\mathrm{CH}), 115.4(\mathrm{C}), 49.4\left(\mathrm{CH}_{2}\right), 21.6\left(\mathrm{CH}_{3}\right)$. HRMS (ESI $\left.{ }^{+}\right)$: Calcd for ${ }^{12} \mathrm{C}_{29}{ }^{1} \mathrm{H}_{20}{ }^{14} \mathrm{~N}_{3}{ }^{16} \mathrm{O}^{32} \mathrm{~S}^{35} \mathrm{Cl}[\mathrm{M}+\mathrm{H}]{ }^{+}:$494.1094; Found: 494.1095.

$N^{8}$-benzyl-2-(4-chlorophenyl)-7-(4-methoxyphenyl)thiazolo[5,4-f]quinazolin-9(8H)-one (4dc)

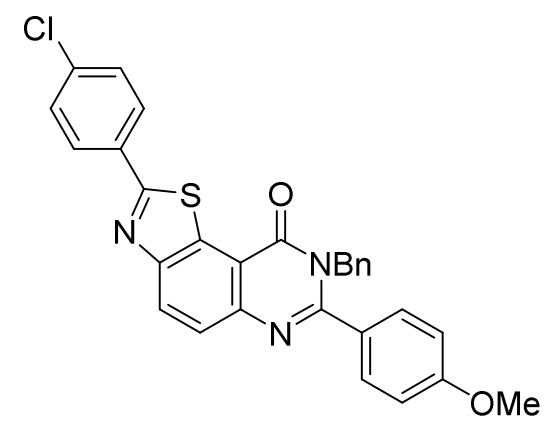

Yield: 33\%; 45 mg; Yellow solid; Rf: 0.82 (DCM/EtOAc, 4/1, v/v); mp 248-251 ${ }^{\circ} \mathrm{C}$; IR (neat) $v_{\max }$ 3052, 3006, 2921, 2850, 1726, 1577, 1511, 1449, 1346, 1306, 1257, 1174, 1088, $1019 \mathrm{~cm}^{-1}$; ${ }^{1} \mathrm{H} \mathrm{NMR}\left(\mathrm{CDCl}_{3}, 25^{\circ} \mathrm{C}, 300 \mathrm{MHz}\right): \delta_{H} 8.43(1 \mathrm{H}, \mathrm{d}, J=8.6 \mathrm{~Hz}), 8.13(2 \mathrm{H}, \mathrm{d}, J=8.4 \mathrm{~Hz}), 7.91(1 \mathrm{H}$, $\mathrm{d}, J=8.7 \mathrm{~Hz}), 7.50(2 \mathrm{H}, \mathrm{d}, J=8.2 \mathrm{~Hz}),, 7.38(2 \mathrm{H}, \mathrm{d}, J=8.5 \mathrm{~Hz}), 7.31-7.16(3 \mathrm{H}, \mathrm{m}), 7.10-6.97$ $(2 \mathrm{H}, \mathrm{m}), 6.95(2 \mathrm{H}, \mathrm{d}, J=8.2 \mathrm{~Hz}), 5.43(2 \mathrm{H}, \mathrm{s}), 3.87(3 \mathrm{H}, \mathrm{s}) ;{ }^{13} \mathrm{C}\left\{{ }^{1} \mathrm{H}\right\} \mathrm{NMR}\left(\mathrm{CDCl}_{3}, 25{ }^{\circ} \mathrm{C}, 75.4\right.$ $\mathrm{MHz}$ ): $\delta_{\mathrm{C}} 170.1$ (C), 161.6 (C), 161.2 (C), 156.3 (C), 153.3 (C), 150.5 (C), 145.9 (C), 137.3 (C), 136.4 (C), 132.3 (C), $130.0(2 \times \mathrm{CH}), 129.6(2 \times \mathrm{CH}), 129.5(\mathrm{CH}), 129.3(\mathrm{C}), 128.8(4 \times \mathrm{CH}), 128.4$ (C), $127.8(\mathrm{CH}), 127.1(2 \times \mathrm{CH}), 126.5(\mathrm{CH}), 114.2(2 \times \mathrm{CH}), 55.7\left(\mathrm{CH}_{3}\right), 49.5\left(\mathrm{CH}_{2}\right)$. HRMS $\left.(\mathrm{ESI})^{+}\right)$: Calcd for ${ }^{12} \mathrm{C}_{29}{ }^{1} \mathrm{H}_{20}{ }^{14} \mathrm{~N}_{3}{ }^{16} \mathrm{O}_{2}{ }^{32} \mathrm{~S}^{35} \mathrm{Cl}[\mathrm{M}+\mathrm{H}]^{+}:$510.1043; Found: 510.1055. 


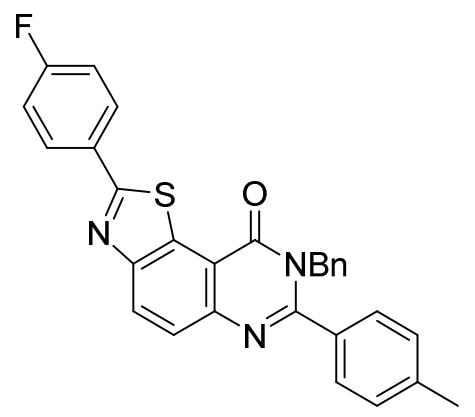

Yield: 59\%; 76 mg; Yellow solid; Rf: 0.92 (DCM/EtOAc, 4/1, v/v); mp 204-207 ${ }^{\circ} \mathrm{C}$; IR (neat) $v_{\max }$ 3064, 3031, 2923, 2854, 1662, 1576, 1514, 1453, 1348, 1230, 1156, 1071, 1029, $969 \mathrm{~cm}^{-1} ;{ }^{1} \mathrm{H}$ $\operatorname{NMR}\left(\mathrm{CDCl}_{3}, 25^{\circ} \mathrm{C}, 300 \mathrm{MHz}\right): \delta_{H} 8.42(1 \mathrm{H}, \mathrm{d}, J=8.8 \mathrm{~Hz}), 8.18(2 \mathrm{H}, \mathrm{dd}, J=8.6,5.3 \mathrm{~Hz}), 7.89$ $(1 \mathrm{H}, \mathrm{d}, J=8.8 \mathrm{~Hz}), 7.32(2 \mathrm{H}, \mathrm{d}, J=7.9 \mathrm{~Hz}), 7.26-7.13(7 \mathrm{H}, \mathrm{m}), 7.07-6.89(2 \mathrm{H}, \mathrm{m}), 5.41(2 \mathrm{H}$, s), $2.43(3 \mathrm{H}, \mathrm{s}) ;{ }^{13} \mathrm{C}\left\{{ }^{1} \mathrm{H}\right\} \operatorname{NMR}\left(\mathrm{CDCl}_{3}, 25^{\circ} \mathrm{C}, 75.4 \mathrm{MHz}\right): \delta_{C} 170.3(\mathrm{C}), 164.7(\mathrm{C}, \mathrm{d}, J=252.3 \mathrm{~Hz})$, 161.5 (C), 156.4 (C), 153.3 (C), 146.0 (C), 140.6 (C), 136.4 (C), 132.3 (C), 132.0 (C), 130.2 (C, d, $J=3.3 \mathrm{~Hz}), 129.7(2 \times \mathrm{CH}, \mathrm{d}, J=8.5 \mathrm{~Hz}), 129.5(2 \times \mathrm{CH}), 129.3(\mathrm{CH}), 128.8(2 \times \mathrm{CH}), 128.3(2 \times$ $\mathrm{CH}), 127.7(\mathrm{CH}), 127.2(2 \times \mathrm{CH}), 126.5(\mathrm{CH}), 116.5(2 \times \mathrm{CH}, \mathrm{d}, J=22.3 \mathrm{~Hz}), 115.4(\mathrm{C}), 49.4$ $\left(\mathrm{CH}_{2}\right)$, $21.6\left(\mathrm{CH}_{3}\right)$. HRMS $\left(\mathrm{ESI}^{+}\right)$: Calcd for ${ }^{12} \mathrm{C}_{29}{ }^{1} \mathrm{H}_{21}{ }^{14} \mathrm{~N}_{3}{ }^{16} \mathrm{O}^{32} \mathrm{~S}^{19} \mathrm{~F}[\mathrm{M}+\mathrm{H}]{ }^{+}$: 478.1389; Found: 478.1387.

4-( $N^{8}$-benzyl-7-(4-fluorophenyl)-9-oxo-8,9-dihydrothiazolo[5,4-f]quinazolin-2-yl)benzonitrile $(4 \mathrm{fa})$

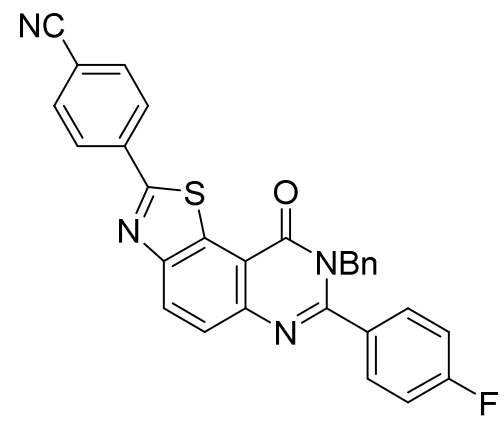

Yield: 69\%; $91 \mathrm{mg}$; Yellow solid; Rf: 0.91 (DCM/EtOAc, 4/1, v/v); mp 276-279 ${ }^{\circ} \mathrm{C}$; IR (neat) $v_{\max }$ $3019,2922,2230,1655,1572,1509,1440,1342,1233,1149,1062,1013 \mathrm{~cm}^{-1} ;{ }^{1} \mathrm{H}$ NMR $\left(\mathrm{CDCl}_{3}, 25^{\circ} \mathrm{C}, 300 \mathrm{MHz}\right): \delta_{H} 8.49(1 \mathrm{H}, \mathrm{d}, J=8.8 \mathrm{~Hz}), 8.31(2 \mathrm{H}, \mathrm{d}, J=8.2 \mathrm{~Hz}), 7.93(1 \mathrm{H}, \mathrm{d}, J=8.8$ $\mathrm{Hz}), 7.82(2 \mathrm{H}, \mathrm{d}, J=8.2 \mathrm{~Hz}), 7.40(2 \mathrm{H}, \mathrm{dd}, J=8.5,5.3 \mathrm{~Hz}), 7.26-7.21(3 \mathrm{H}, \mathrm{m}), 7.13(2 \mathrm{H}, \mathrm{t}, J=$ $8.4 \mathrm{~Hz}), 7.05-6.89(2 \mathrm{H}, \mathrm{m}), 5.40(2 \mathrm{H}, \mathrm{s}) ;{ }^{13} \mathrm{C}\left\{{ }^{1} \mathrm{H}\right\} \mathrm{NMR}\left(\mathrm{CDCl}_{3}, 25^{\circ} \mathrm{C}, 75.4 \mathrm{MHz}\right): \delta_{\mathrm{C}} 169.0(\mathrm{C})$, 161.4 (C), 160.5 (C, d, J = 250.7 Hz), 155.7 (C), 153.4 (C), 146.3 (C), 137.6 (C), 136.1 (C), 134.2 (C), $133.1(2 \times \mathrm{CH}), 132.5(\mathrm{C}), 131.1(\mathrm{C}, \mathrm{d}, J=4.8 \mathrm{~Hz}), 130.5(2 \times \mathrm{CH}, \mathrm{d}, J=8.8 \mathrm{~Hz}), 130.0(\mathrm{CH})$, $128.9(2 \times \mathrm{CH}), 128.0(2 \times \mathrm{CH}), 128.0(\mathrm{CH}), 127.0(2 \times \mathrm{CH}), 127.0(\mathrm{CH}), 116.1(2 \times \mathrm{CH}, \mathrm{d}, J=22.0$ $\mathrm{Hz}), 115.5$ (C), 114.4 (C), $49.4\left(\mathrm{CH}_{2}\right)$. HRMS (ESI $\left.{ }^{+}\right)$: Calcd for ${ }^{12} \mathrm{C}_{29}{ }^{1} \mathrm{H}_{18}{ }^{14} \mathrm{~N}_{4}{ }^{16} \mathrm{O}^{32} \mathrm{~S}^{19} \mathrm{~F}[\mathrm{M}+\mathrm{H}]^{+}$: 489.1185; Found: 489.1178. 


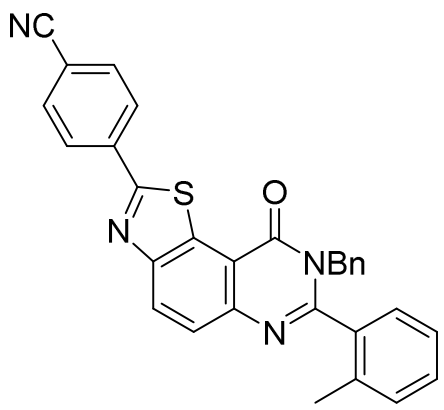

Yield: 78\%; 102 mg; Yellow solid; Rf: 0.92 (DCM/EtOAc, 4/1, v/v); mp 139-142 ${ }^{\circ} \mathrm{C}$; IR (neat) $v_{\max } 3058,3029,2955,2924,2223,1655,1568,1453,1349,1233,1158,1106,966 \mathrm{~cm}^{-1} ;{ }^{1} \mathrm{H}$ $\operatorname{NMR}\left(\mathrm{CDCl}_{3}, 25^{\circ} \mathrm{C}, 300 \mathrm{MHz}\right): \delta_{H} 8.48(1 \mathrm{H}, \mathrm{d}, J=8.8 \mathrm{~Hz}), 8.32(2 \mathrm{H}, \mathrm{d}, J=8.2 \mathrm{~Hz}), 7.92(1 \mathrm{H}, \mathrm{d}, J$ $=8.8 \mathrm{~Hz}), 7.83(2 \mathrm{H}, \mathrm{d}, J=8.2 \mathrm{~Hz}), 7.46-7.38(1 \mathrm{H}, \mathrm{m}), 7.32-7.26(2 \mathrm{H}, \mathrm{m}), 7.23-7.13(4 \mathrm{H}$, m), $6.96-6.81(2 \mathrm{H}, \mathrm{m}), 5.34(1 \mathrm{H}, \mathrm{d}, J=14.7 \mathrm{~Hz}), 5.25(1 \mathrm{H}, \mathrm{d}, J=14.9 \mathrm{~Hz}), 1.96(3 \mathrm{H}, \mathrm{s}) ;{ }^{13} \mathrm{C}\left\{{ }^{1} \mathrm{H}\right\}$ $\operatorname{NMR}\left(\mathrm{CDCl}_{3}, 25^{\circ} \mathrm{C}, 75.4 \mathrm{MHz}\right.$ ): $\delta_{C} 168.8$ (C), 161.5 (C), 156.2 (C), 153.3 (C), 146.5 (C), 137.7 (C), 136.3 (C), 135.8 (C), 134.4 (C), $133.1(2 \times \mathrm{CH}), 132.5$ (C), 130.9 (CH), $130.3(\mathrm{CH}), 129.9$ $(\mathrm{CH}), 128.6(2 \times \mathrm{CH}), 128.2(\mathrm{CH}), 128.1(\mathrm{CH}), 128.0(4 \times \mathrm{CH}), 127.0(\mathrm{CH}), 126.2(\mathrm{CH}), 118.5(\mathrm{C})$, 115.6 (C), 114.3 (C), $48.7\left(\mathrm{CH}_{2}\right), 19.14\left(\mathrm{CH}_{3}\right)$. HRMS (ESI $\left.{ }^{+}\right)$: Calcd for ${ }^{12} \mathrm{C}_{30}{ }^{1} \mathrm{H}_{21}{ }^{14} \mathrm{~N}_{4}{ }^{16} \mathrm{O}^{32} \mathrm{~S}[\mathrm{M}+$ $\mathrm{H}^{+}$: 485.1436; Found: 485.1428.

\section{General one-pot procedure for the sequential C-H bis-arylation}

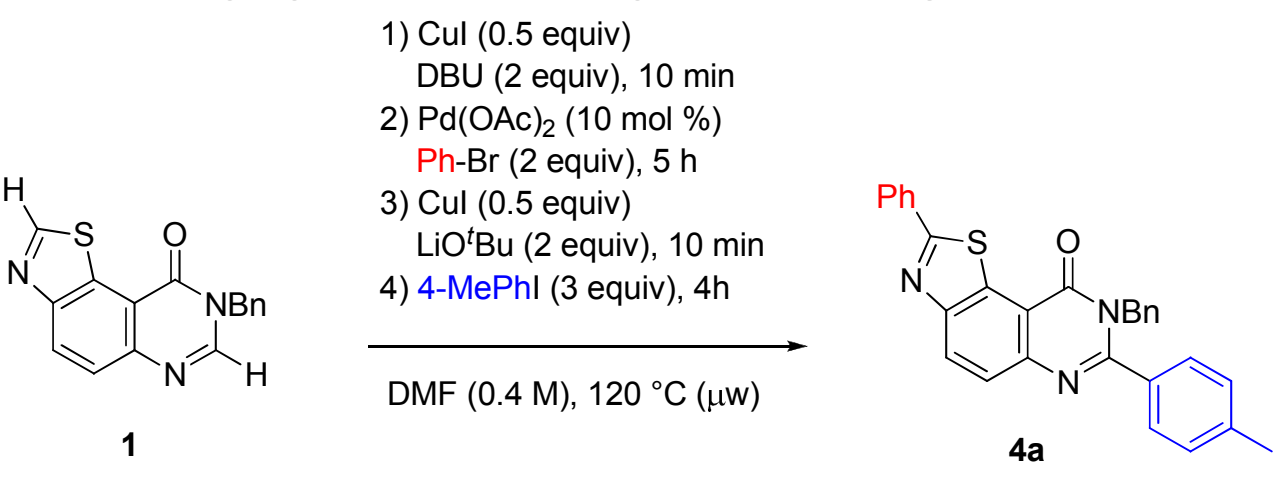

$N^{8}$-benzylthiazolo[5,4-f]quinazolin-9(8H)-one 1 (100 mg, $0.341 \mathrm{mmol}, 1.0$ equiv), copper iodide (33 mg, $0.170 \mathrm{mmol}, 0.5$ equiv) and DBU (101 $\mu \mathrm{L}, 0.682 \mathrm{mmol}, 2.0$ equiv) in dry DMF $(850 \mu \mathrm{L})$ are added to a $2 \mathrm{~mL}$ glass microwave vial. The mixture is stirred under microwave irradiation at $120^{\circ} \mathrm{C}$ for $10 \mathrm{~min}$ ( $2 \mathrm{~min}$ ramp). Then $\mathrm{Pd}(\mathrm{OAc})_{2}(7.6 \mathrm{mg}, 0.034 \mathrm{mmol}, 10 \mathrm{~mol} \%)$ and phenyl bromide ( $71 \mu \mathrm{L}, 0.680 \mathrm{mmol}, 2.0$ equiv) are added to the mixture. The reaction is then stirred under microwave irradiation at $120^{\circ} \mathrm{C}$ for $5 \mathrm{~h}(2 \mathrm{~min}$ ramp). Then copper iodide (33 mg, $0.170 \mathrm{mmol}, 0.5$ equiv) and lithium tert-butoxide ( $55 \mathrm{mg}, 0.542 \mathrm{mmol}, 2.0$ equiv) are added to the mixture. The reaction is then stirred under microwave irradiation at $120^{\circ} \mathrm{C}$ for $10 \mathrm{~min}$ ( $2 \mathrm{~min}$ ramp). And then 4-iodotoluene ( $222 \mathrm{mg}, 1.023 \mathrm{mmol}, 3$ equiv) is added to the mixture. The reaction is then stirred under microwave irradiation at $120{ }^{\circ} \mathrm{C}$ for $4 \mathrm{~h}(2 \mathrm{~min}$ ramp). The resulting solution is diluted with dichloromethane, filtered through cotton and washed three times with dichloromethane. The volatiles are rotary evaporated and the 
crude product is purified by flash chromatography on silica gel with a gradient of petroleum ether /dichloromethane/ethyl acetate (PE/DCM/EtOAc, from $1 / 4 / 0$ to $0 / 1 / 0$ to $0 / 4 / 1, v / v$ ) as eluent to afford the corresponding product.

III. NMR spectroscopic characterization 
$N^{8}$-benzylthiazolo[5,4-f]quinazolin-9(8H)-one (1)

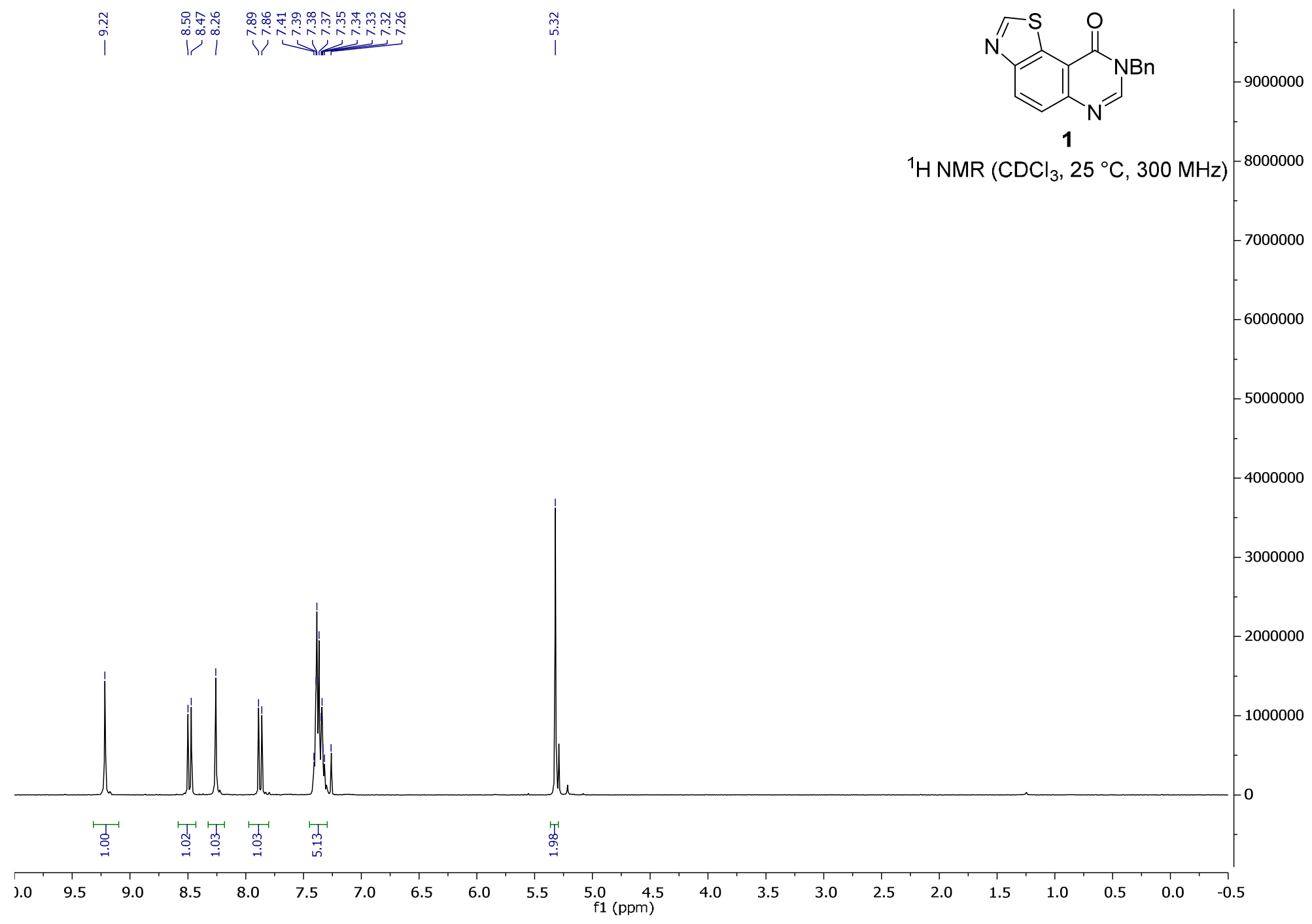




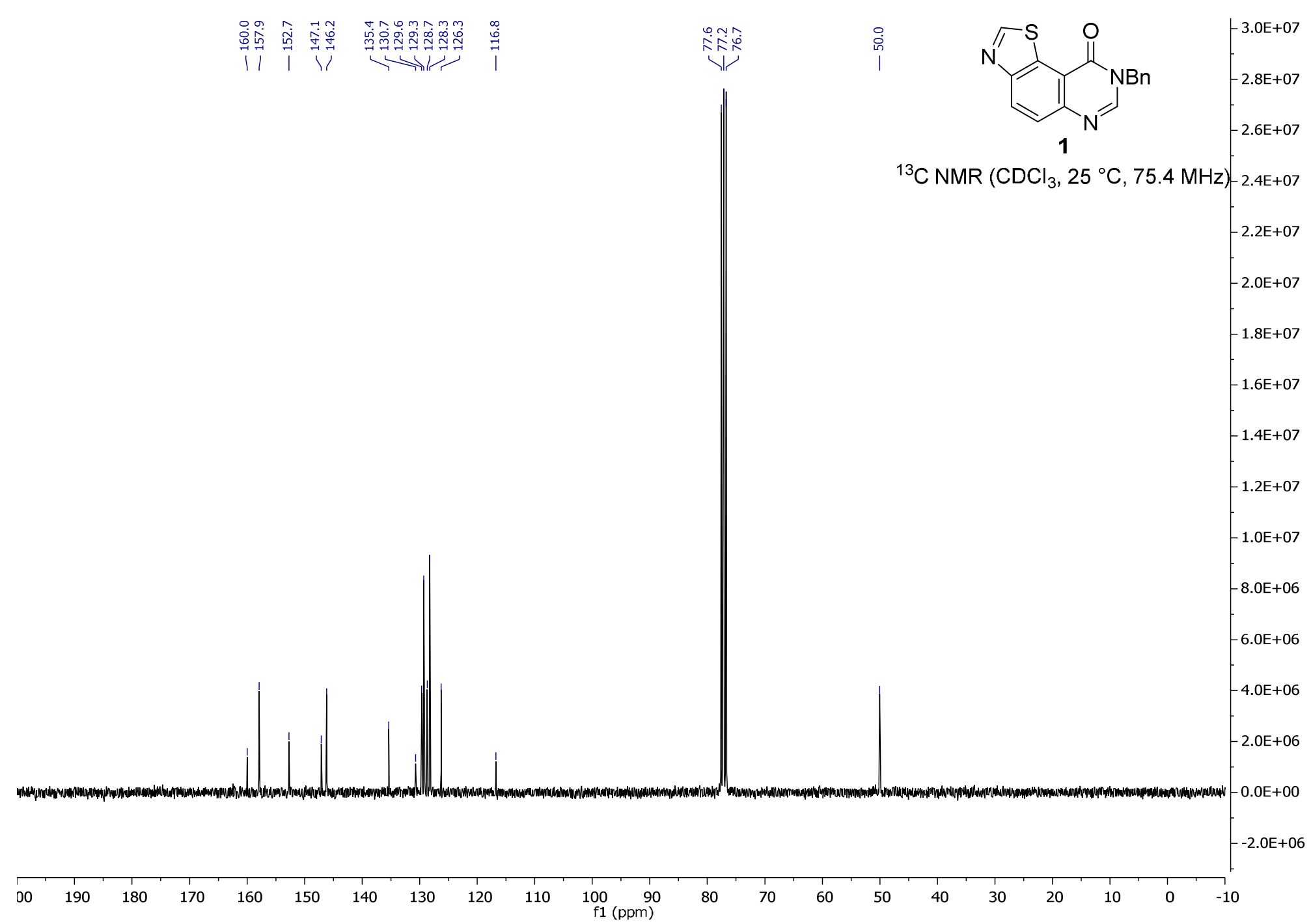


$N^{8}$-benzyl-2-phenylthiazolo[5,4-f]quinazolin-9(8H)-one (2a)

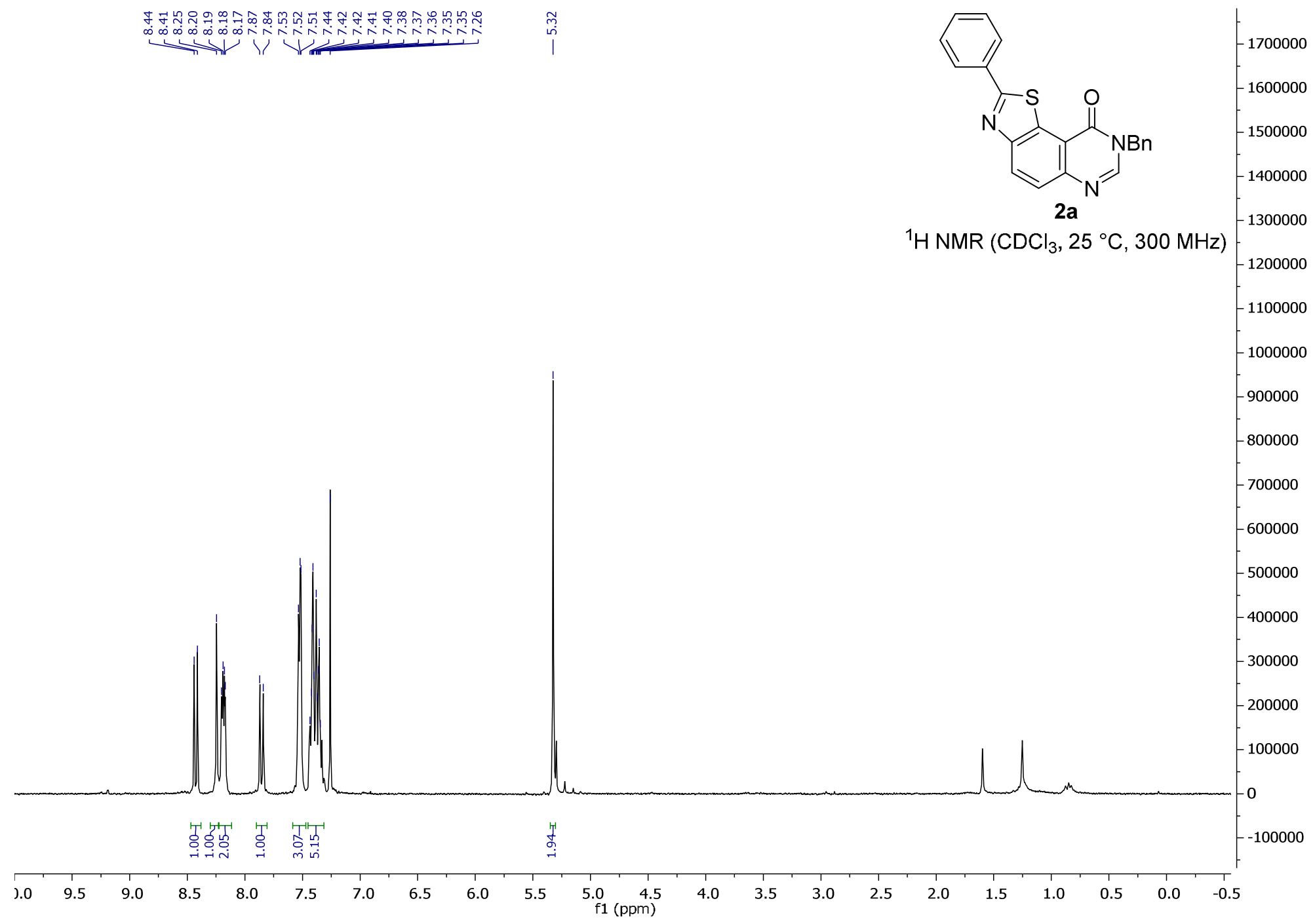




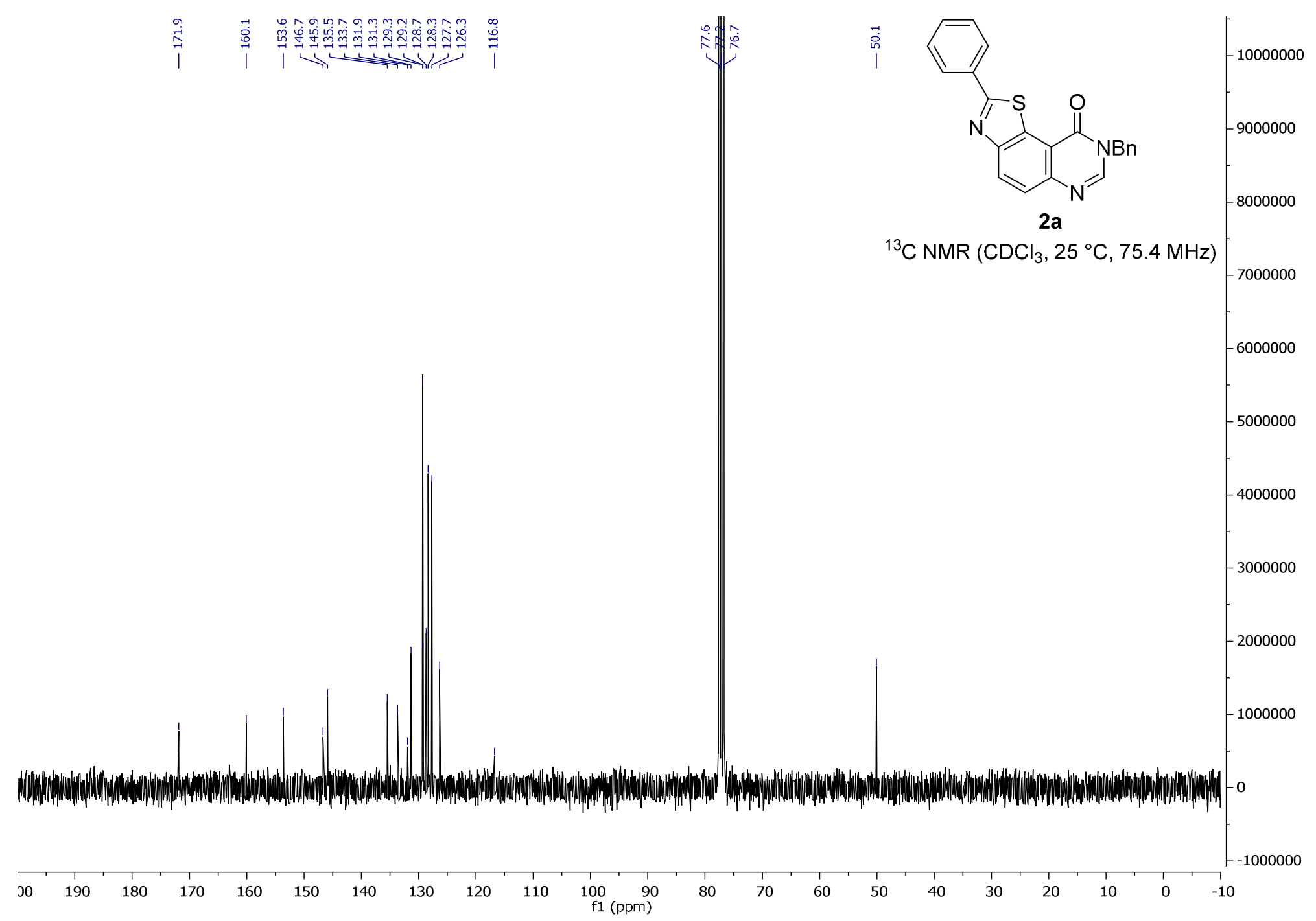


$N^{8}$-benzyl-2-(p-tolyl)thiazolo[5,4-f]quinazolin-9(8H)-one (2b)

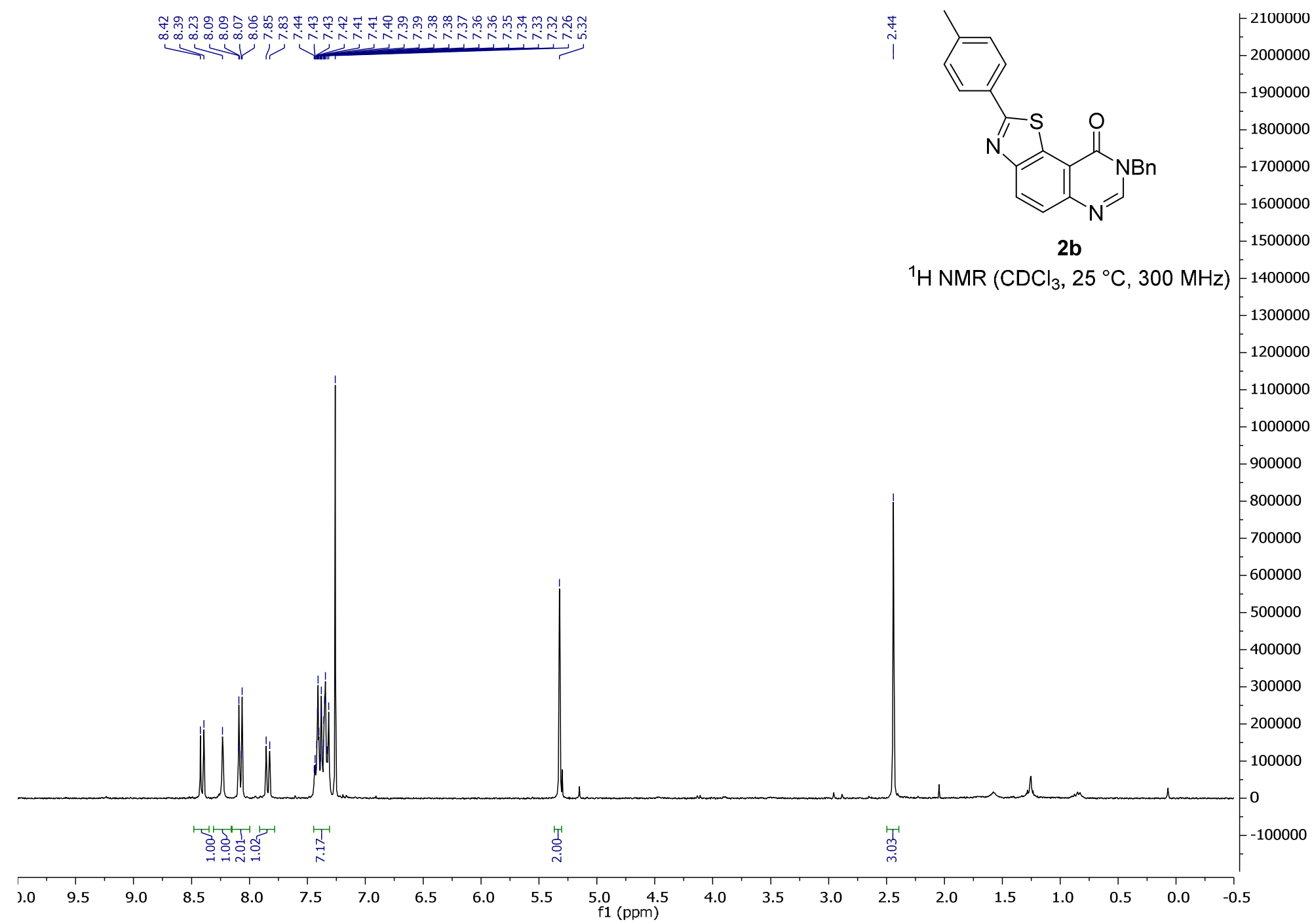




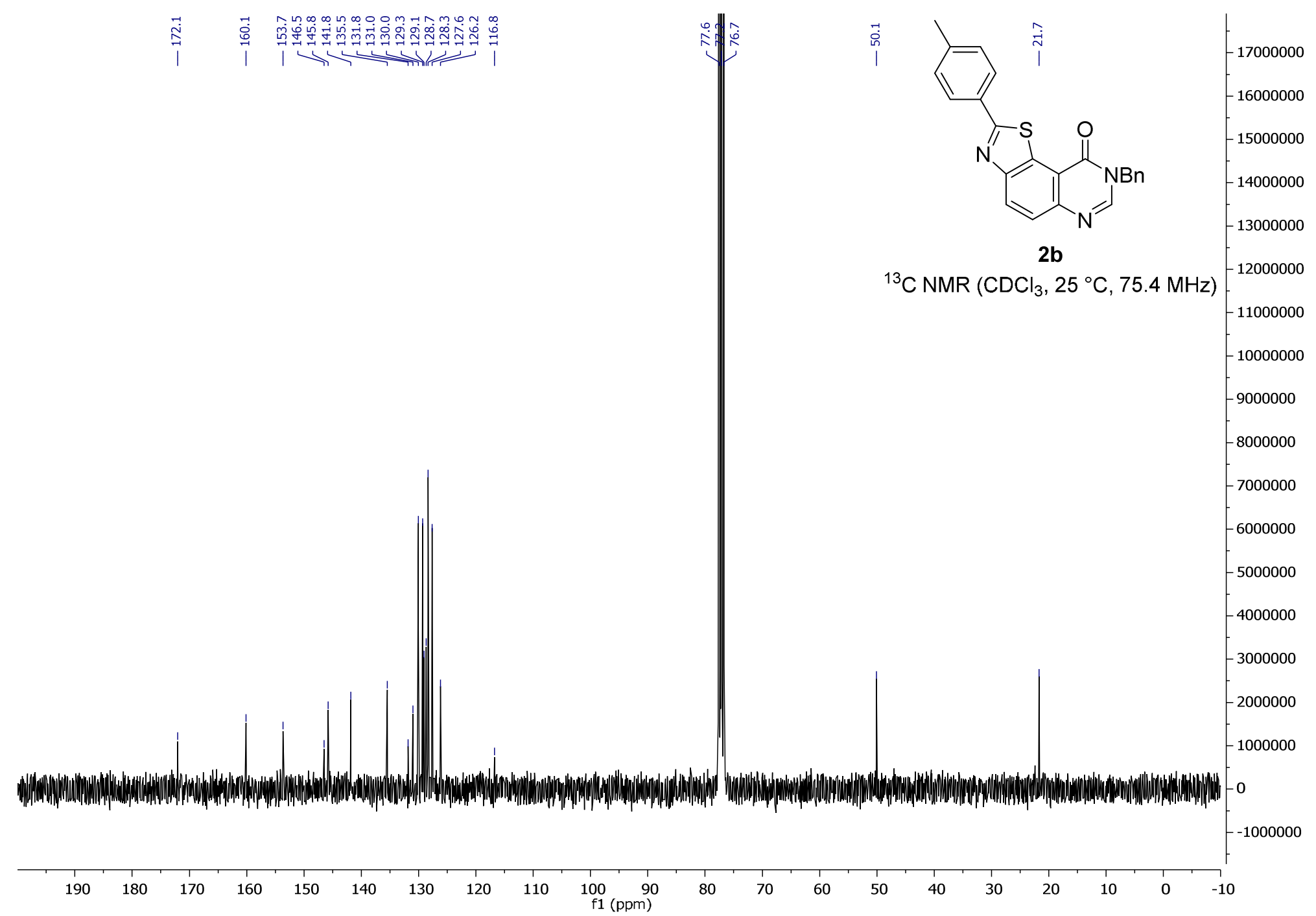


$N^{8}$-benzyl-2-(4-methoxyphenyl)thiazolo[5,4-f]quinazolin-9(8H)-one (2c)

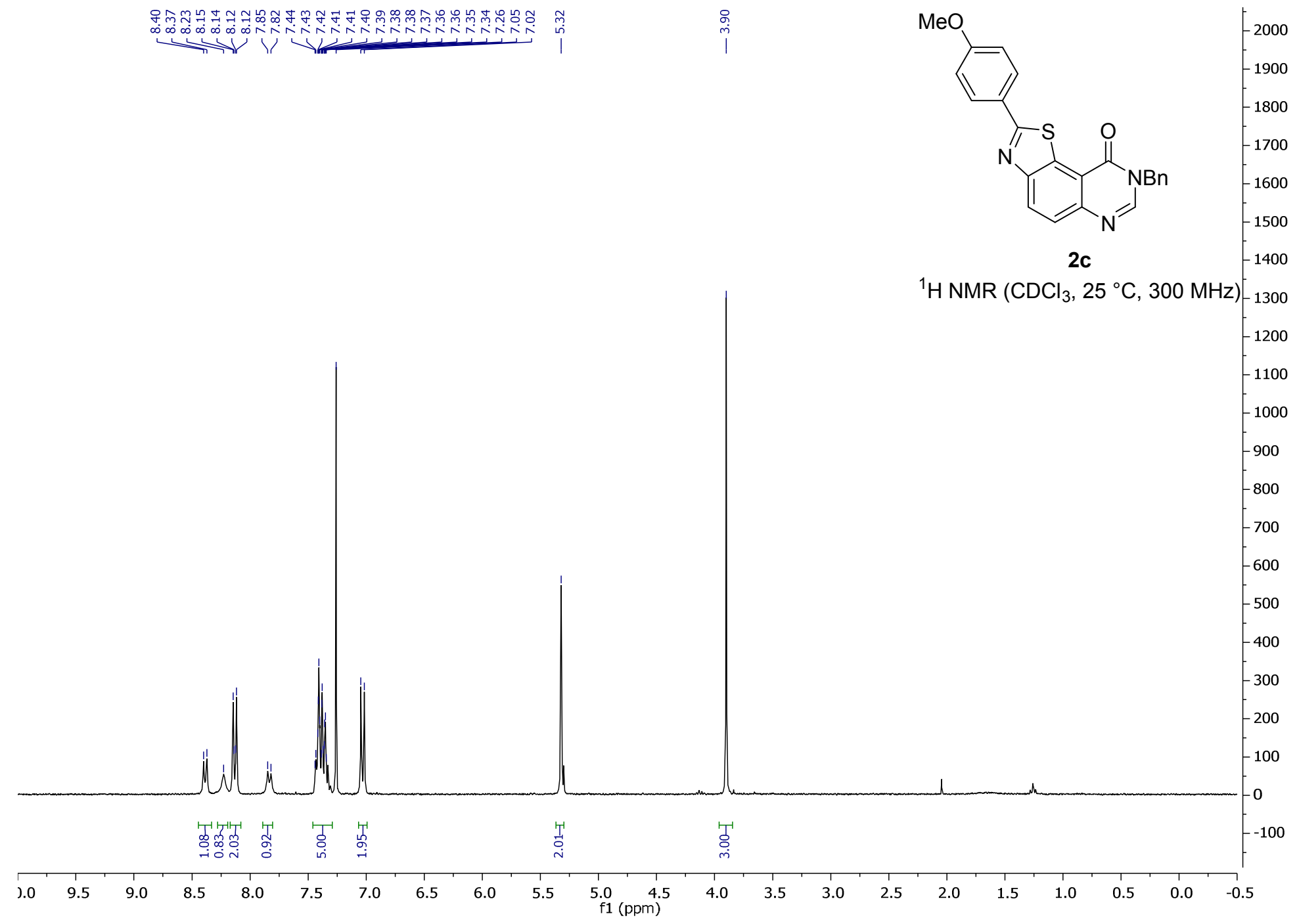




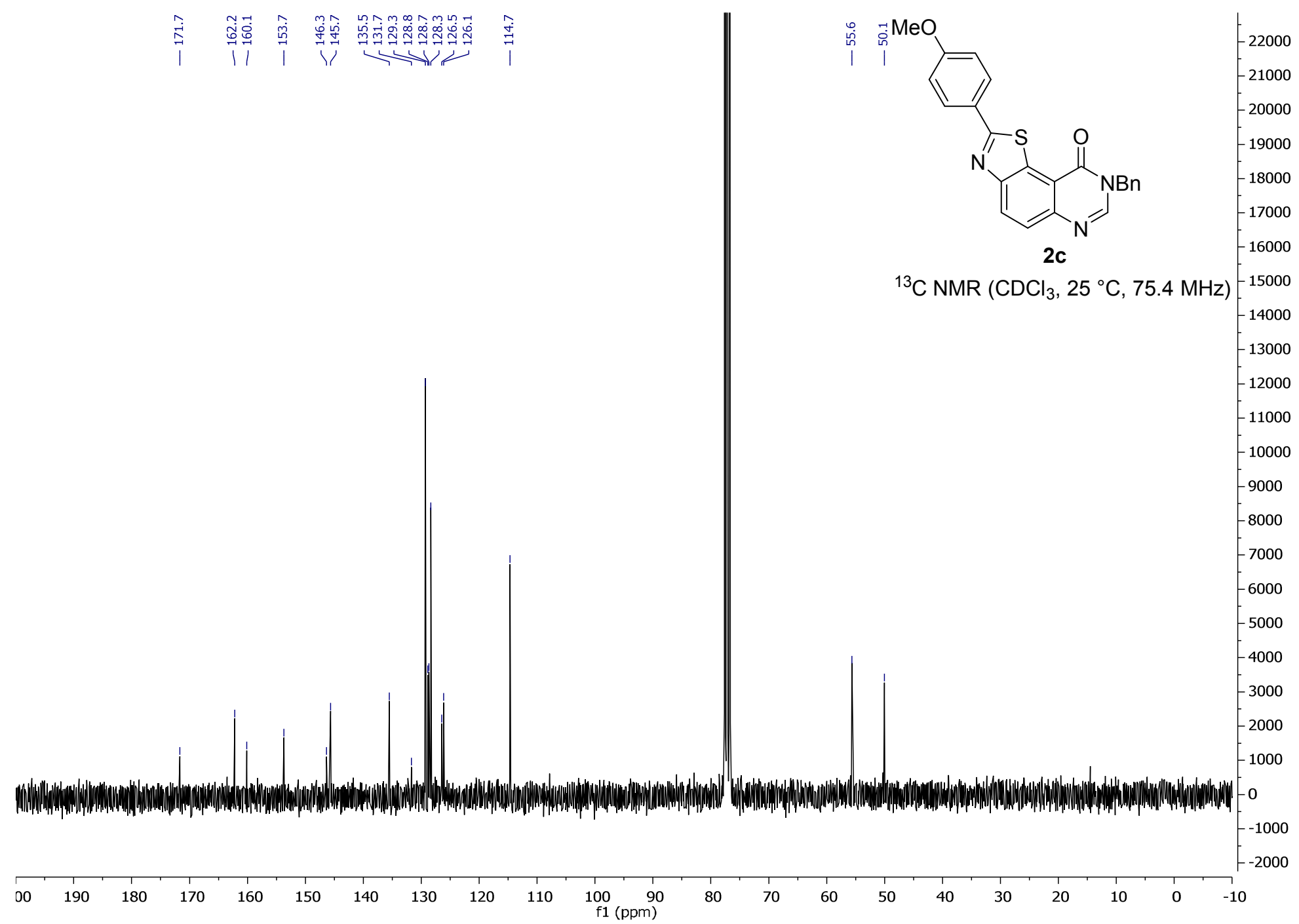


$N^{8}$-benzyl-2-(4-chlorophenyl)thiazolo[5,4-f]quinazolin-9(8H)-one (2d)

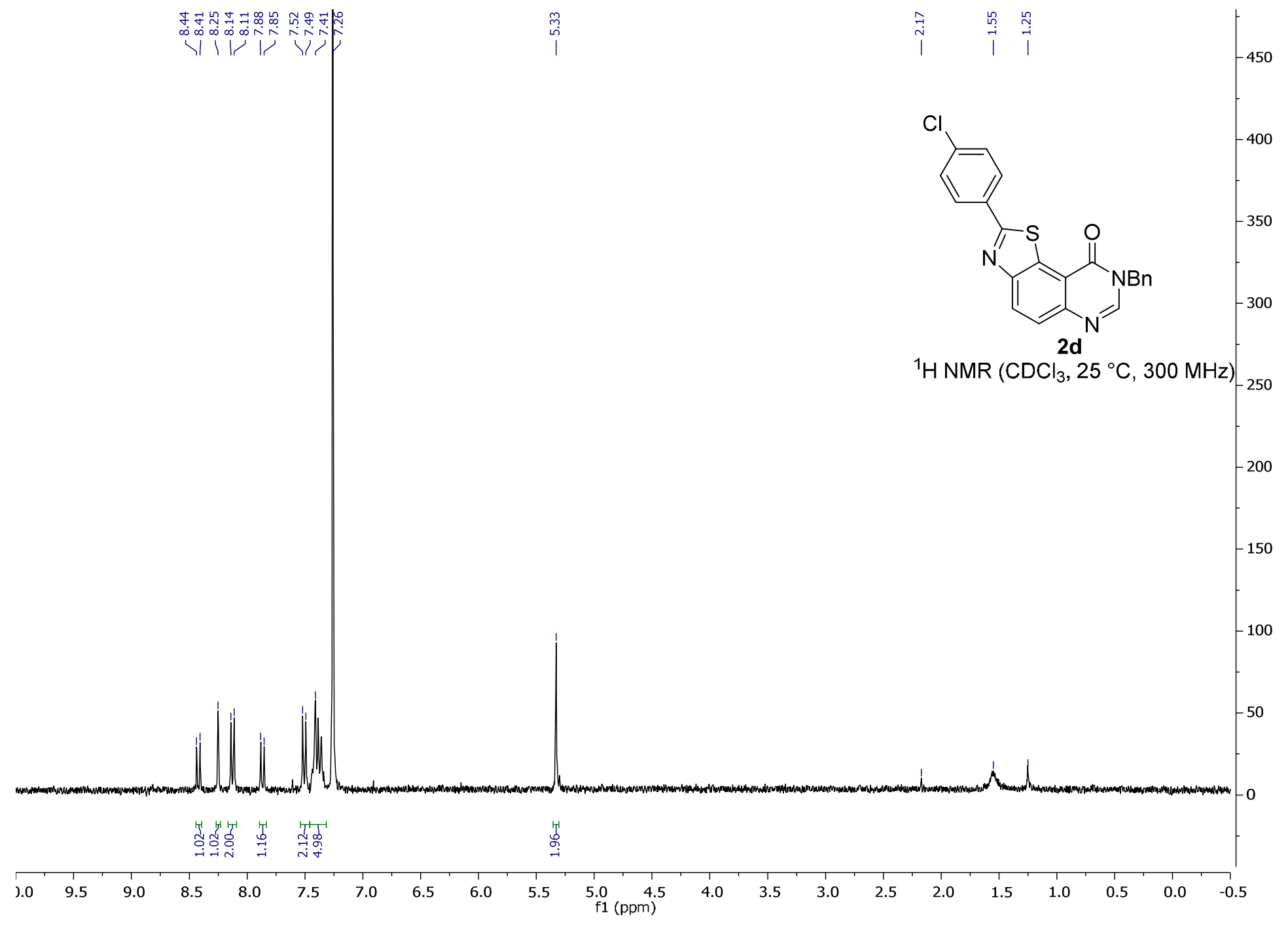




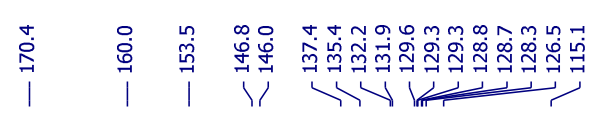

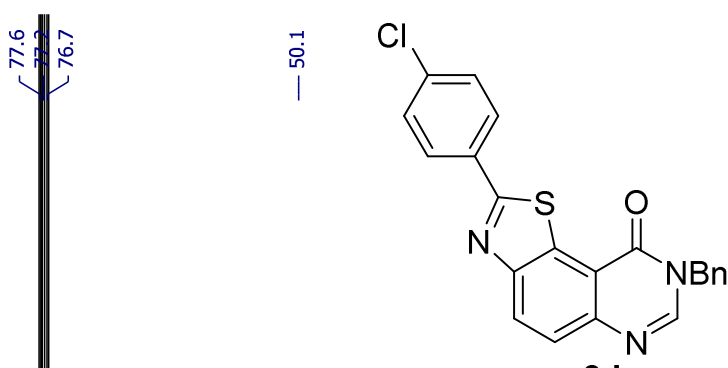

$-45000$

2d

${ }^{13} \mathrm{C}$ NMR $\left(\mathrm{CDCl}_{3}, 25^{\circ} \mathrm{C}, 75.4 \mathrm{MHz}\right)$

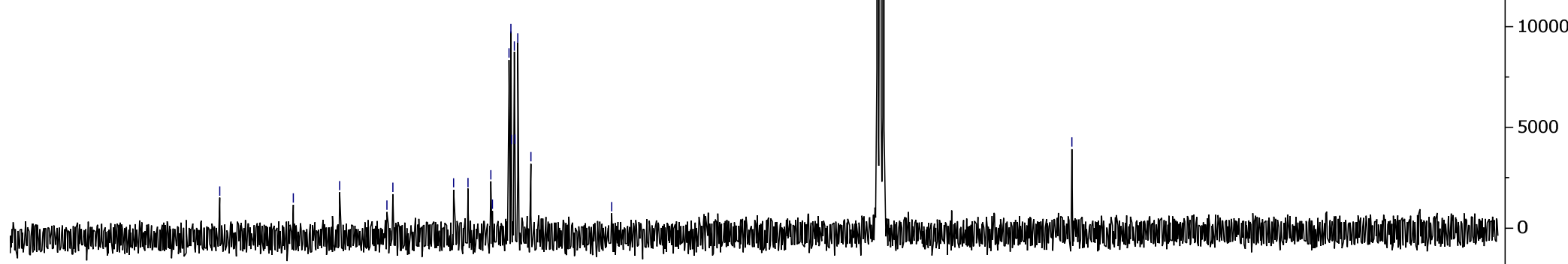

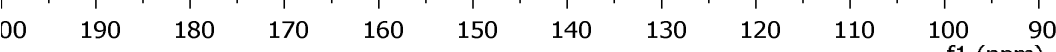


$N^{8}$-benzyl-2-(4-fluorophenyl)thiazolo[5,4-f]quinazolin-9(8H)-one (2e)

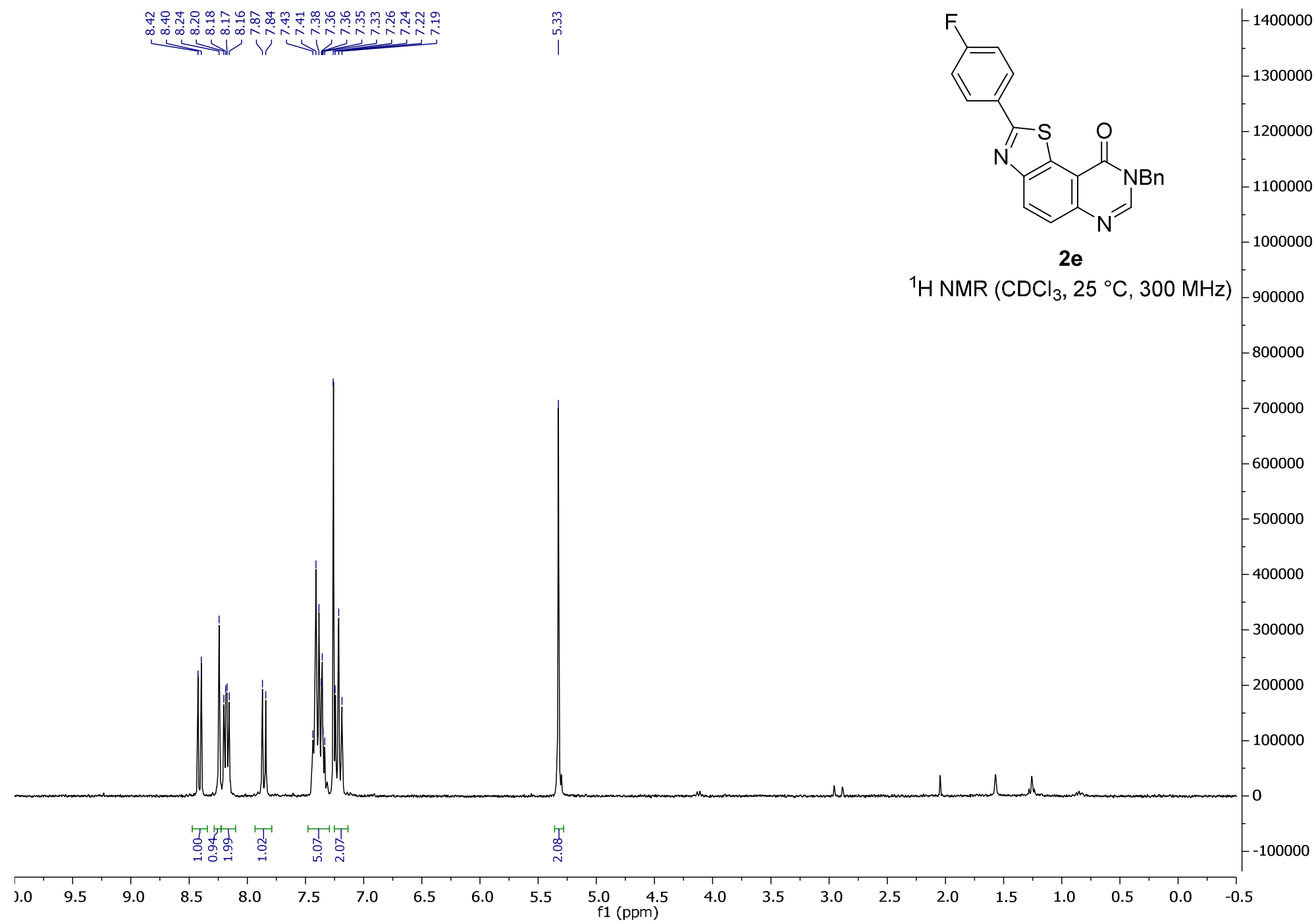




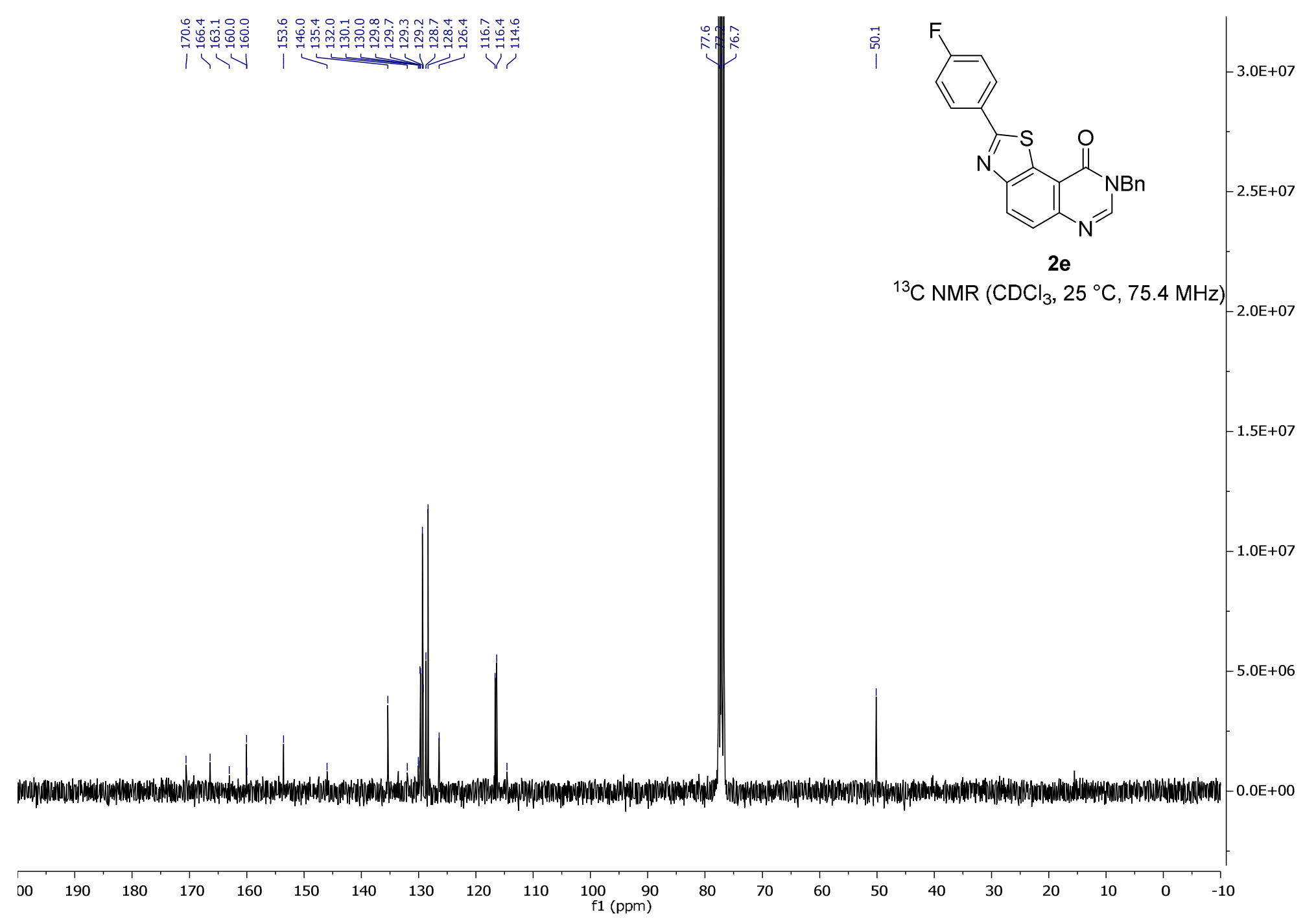


4-( $N^{8}$-benzyl-9-oxo-8,9-dihydrothiazolo[5,4-f]quinazolin-2-yl)benzonitrile (2f)

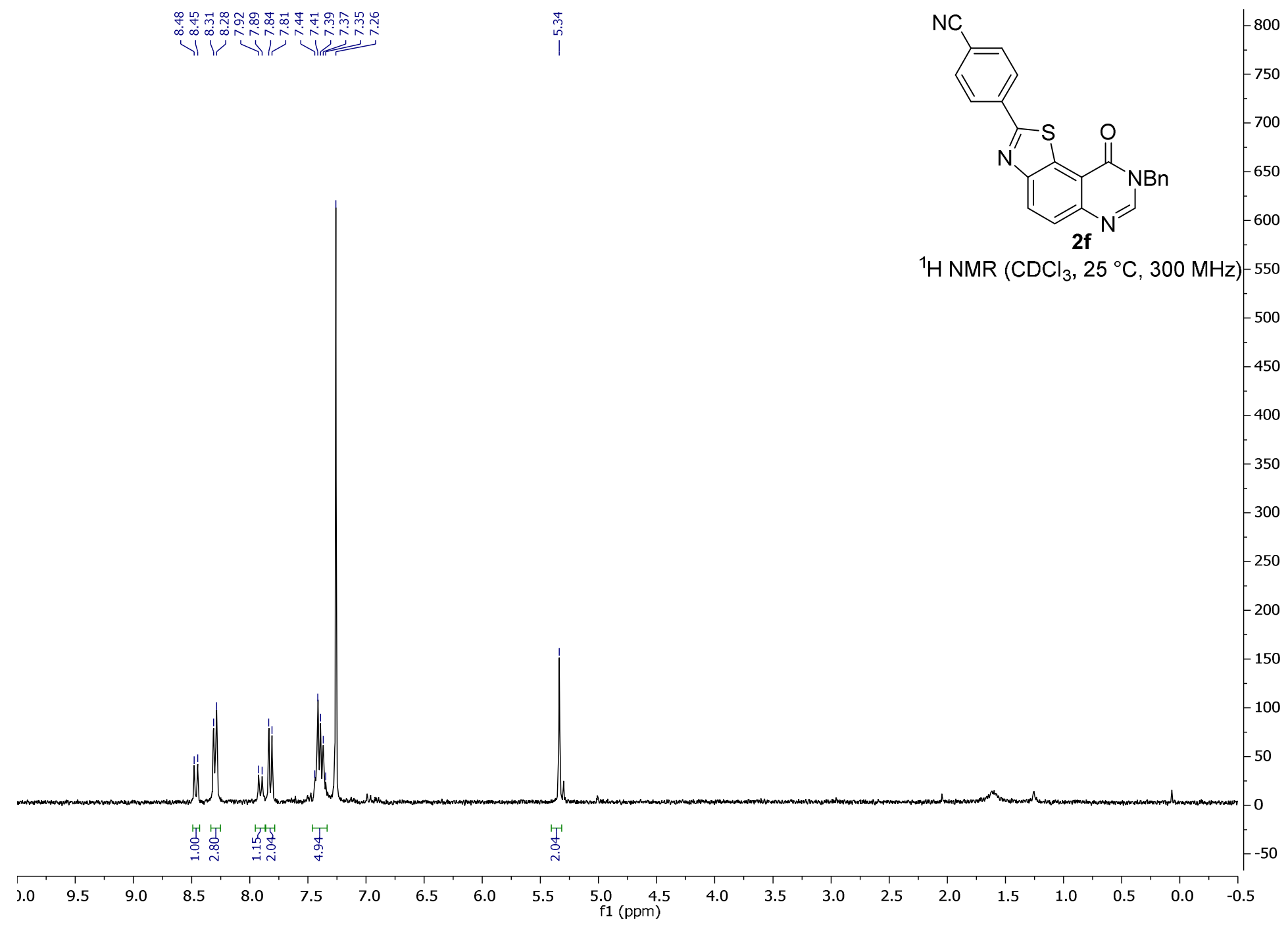




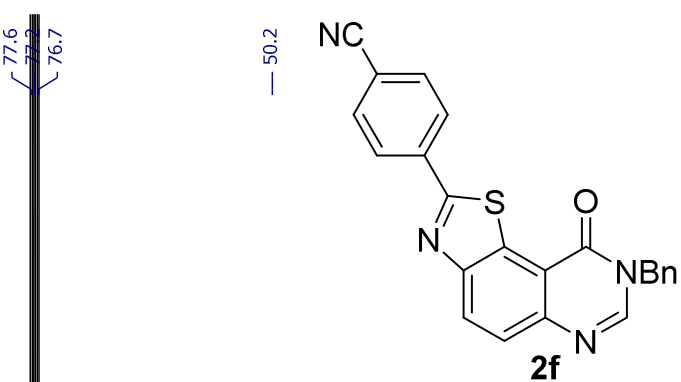

.

140000

$-130000$

$-120000$

$-110000$

${ }^{13} \mathrm{C}$ NMR $\left(\mathrm{CDCl}_{3}, 25^{\circ} \mathrm{C}, 75.4 \mathrm{MHz}\right)-100000$

90000

$-80000$

70000

$\begin{array}{llllllllllll}\text { DO } & 190 & 180 & 170 & 160 & 150 & 140 & 130 & 120 & 110 & 100 & 90\end{array}$

80

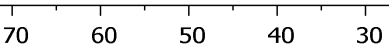

20 
$N^{8}$-benzyl-2-(2,4-dichlorophenyl)thiazolo[5,4-f] quinazolin-9(8H)-one (2g)

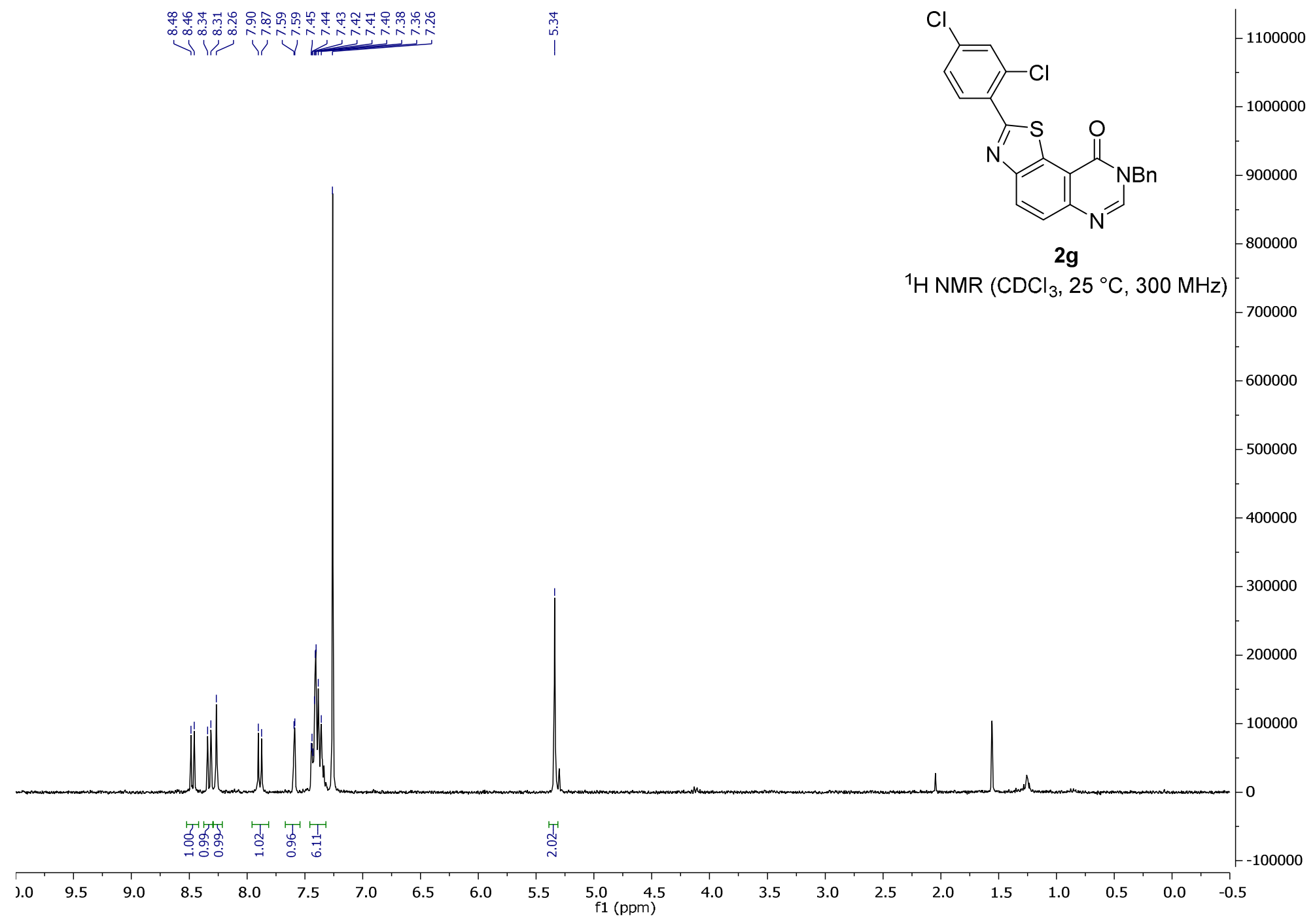




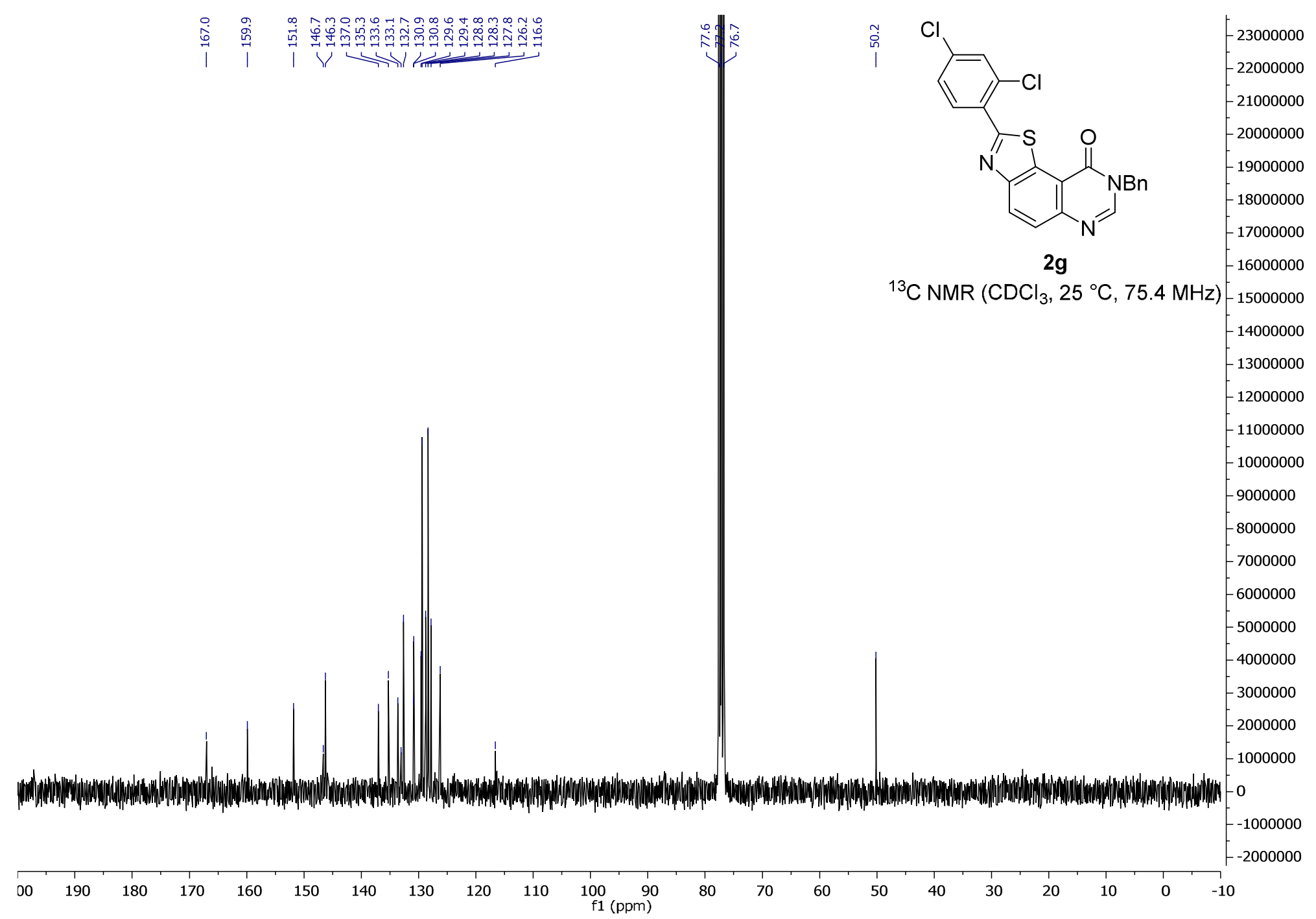


$N^{8}$-benzyl-2-(4-(dimethylamino)phenyl)thiazolo[5,4-f quinazolin-9(8H)-one (2h)

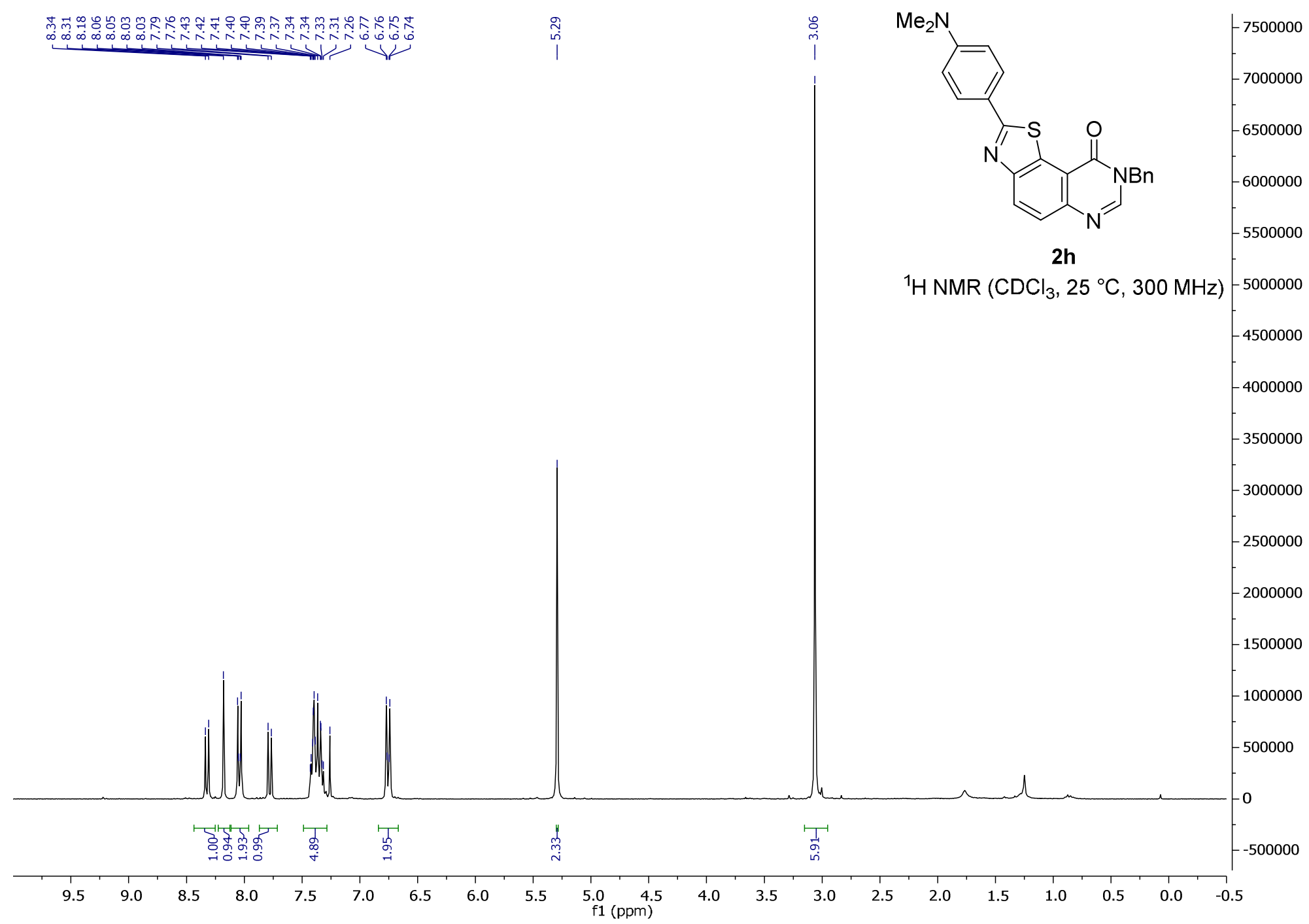




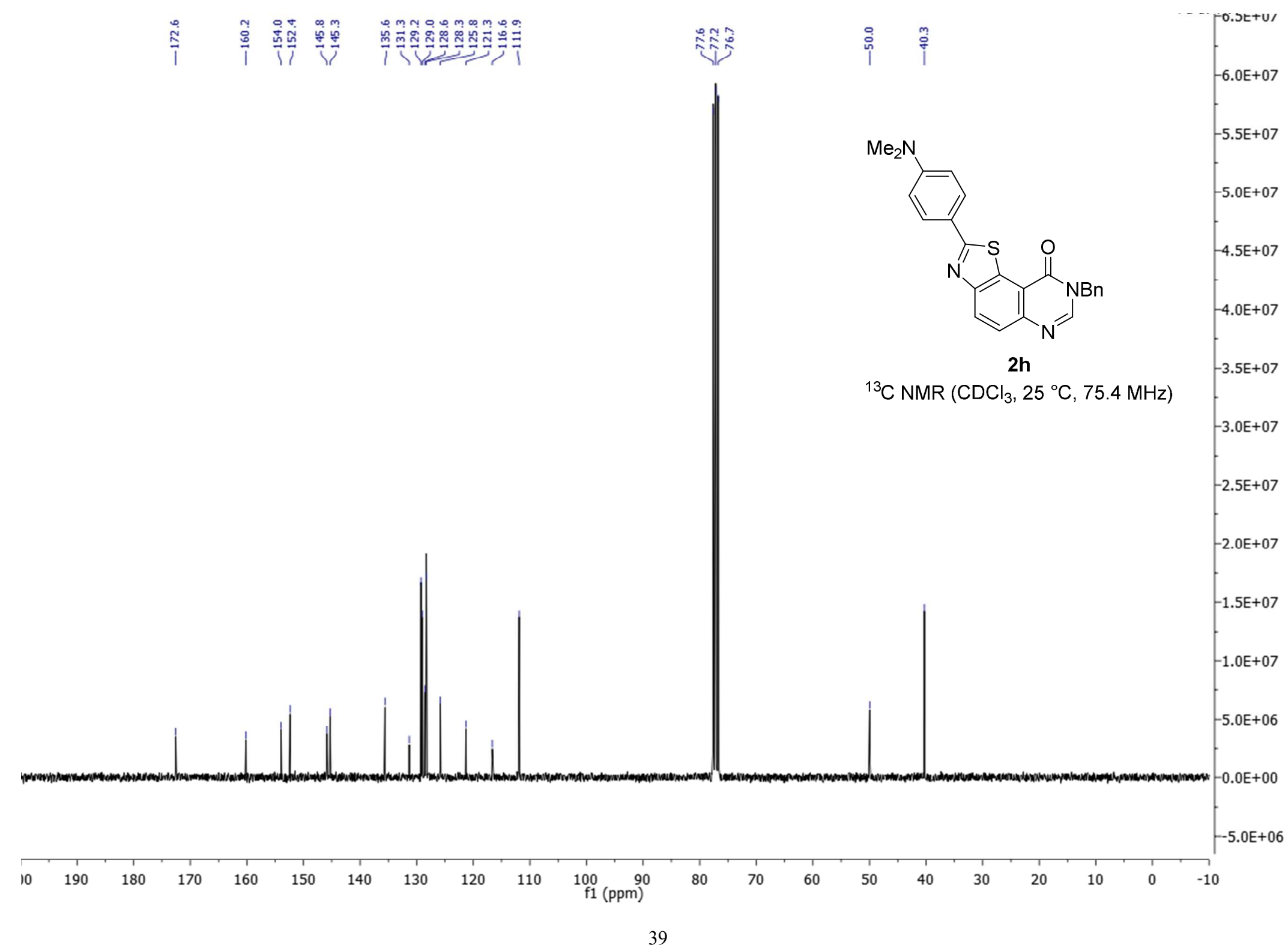


$N^{8}$-benzyl-2-(pyridin-3-yl)thiazolo[5,4-f]quinazolin-9(8H)-one (2i)

V|

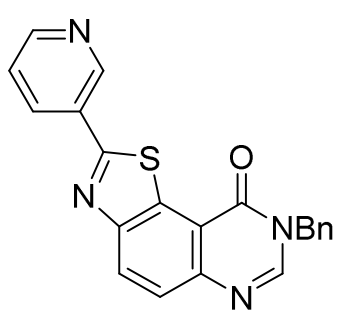

$2 \mathbf{i}$
$-2500000$

$-2400000$

$-2300000$

$-2200000$

$-2100000$

$-2000000$

$-1900000$

$-1800000$

${ }^{1} \mathrm{H}$ NMR $\left(\mathrm{CDCl}_{3}, 25^{\circ} \mathrm{C}, 300 \mathrm{MHz}\right)-1700000$

$-1600000$

$-1500000$

$-1400000$

1300000

$-1200000$

$-1100000$

1000000

$-900000$

$-800000$

$-700000$

$-600000$

$-500000$

$-400000$

$-300000$

$-200000$

$-100000$

0

$-100000$

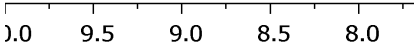

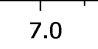

$6.5 \quad 6$

$\begin{array}{lr}5.0 & 4.5 \\ \mathrm{f} 1(\mathrm{ppm})\end{array}$ 


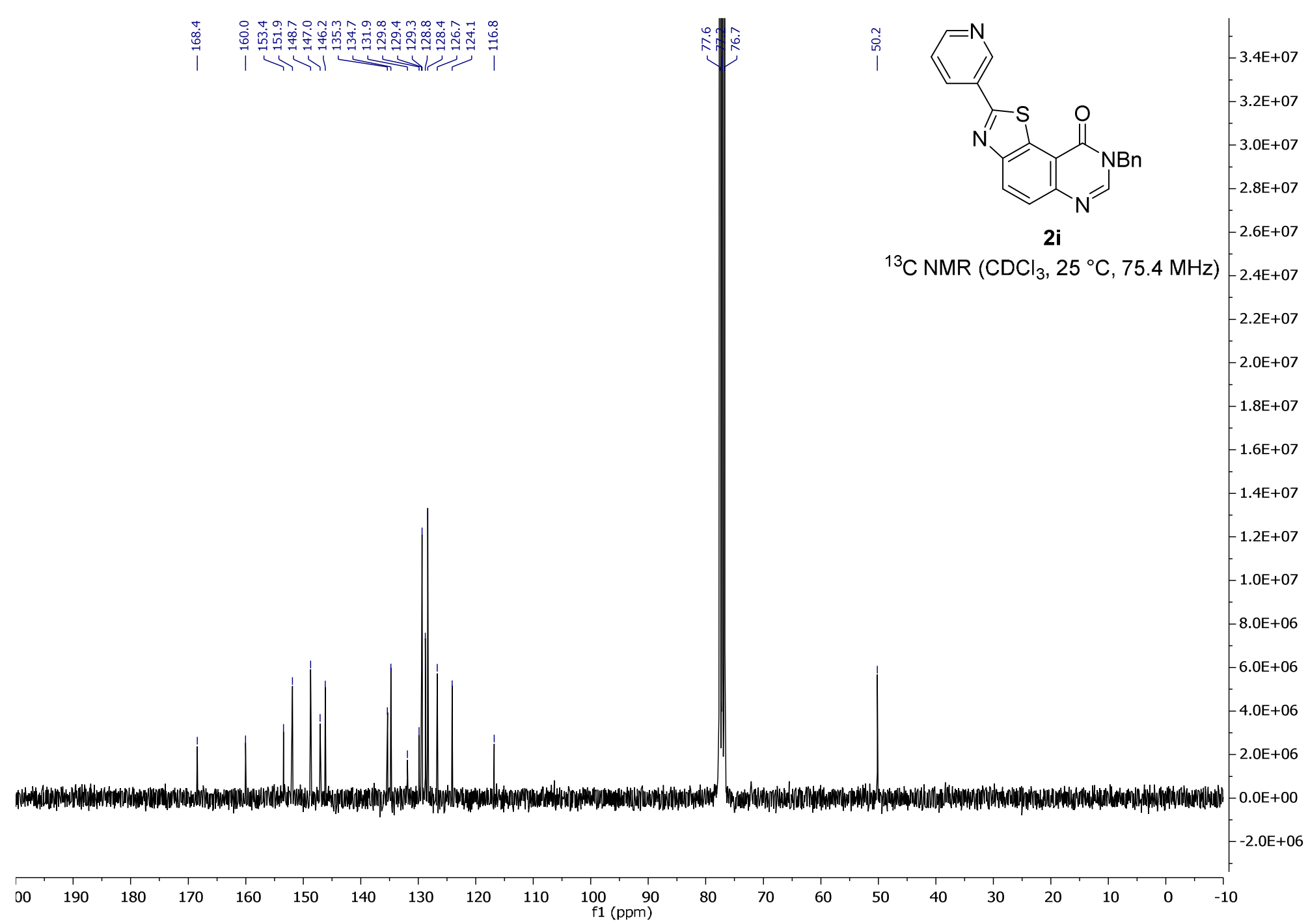


$N^{8}$-benzyl-2-(pyrazin-2-yl)thiazolo[5,4-f]quinazolin-9(8H)-one (2j)

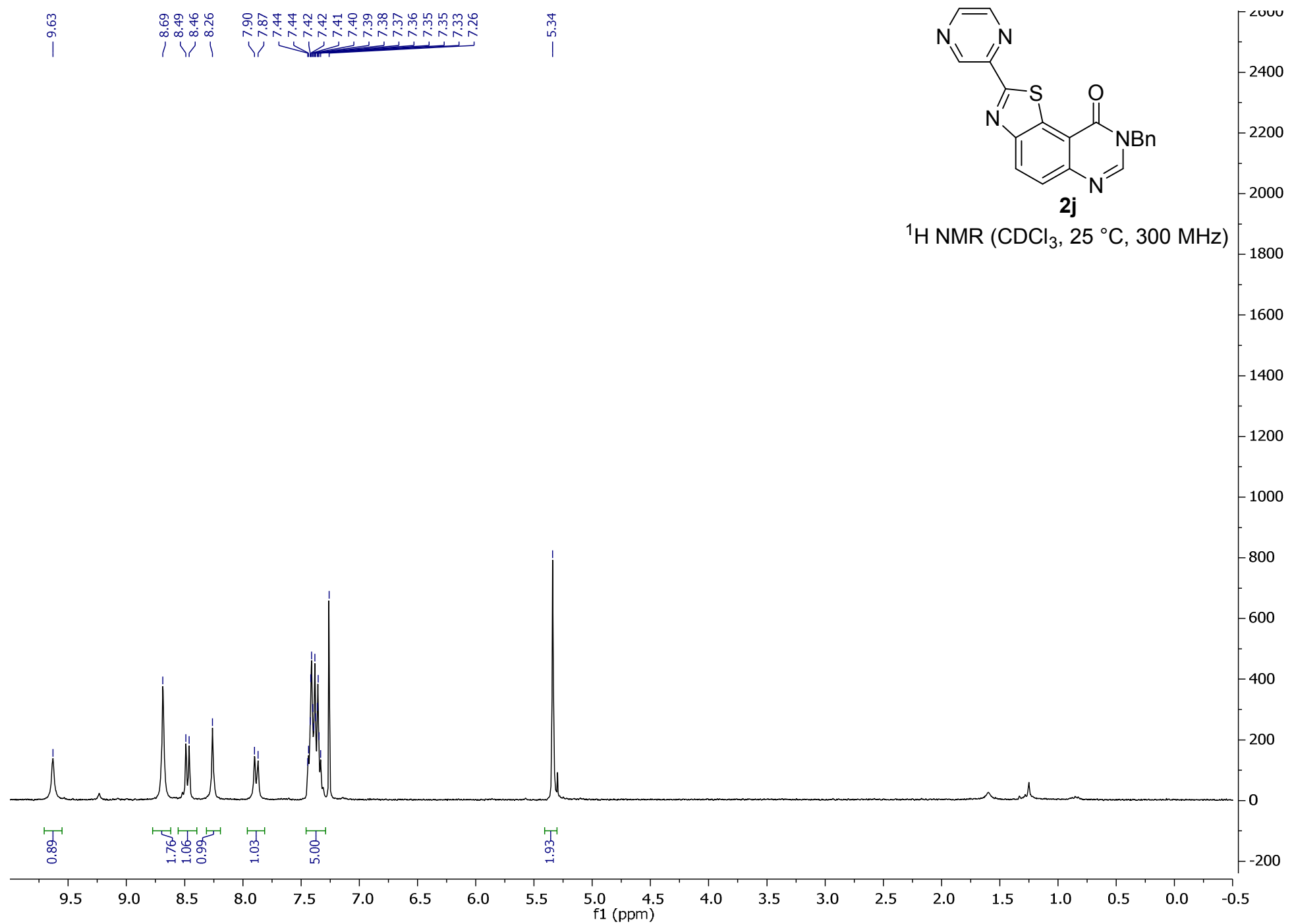




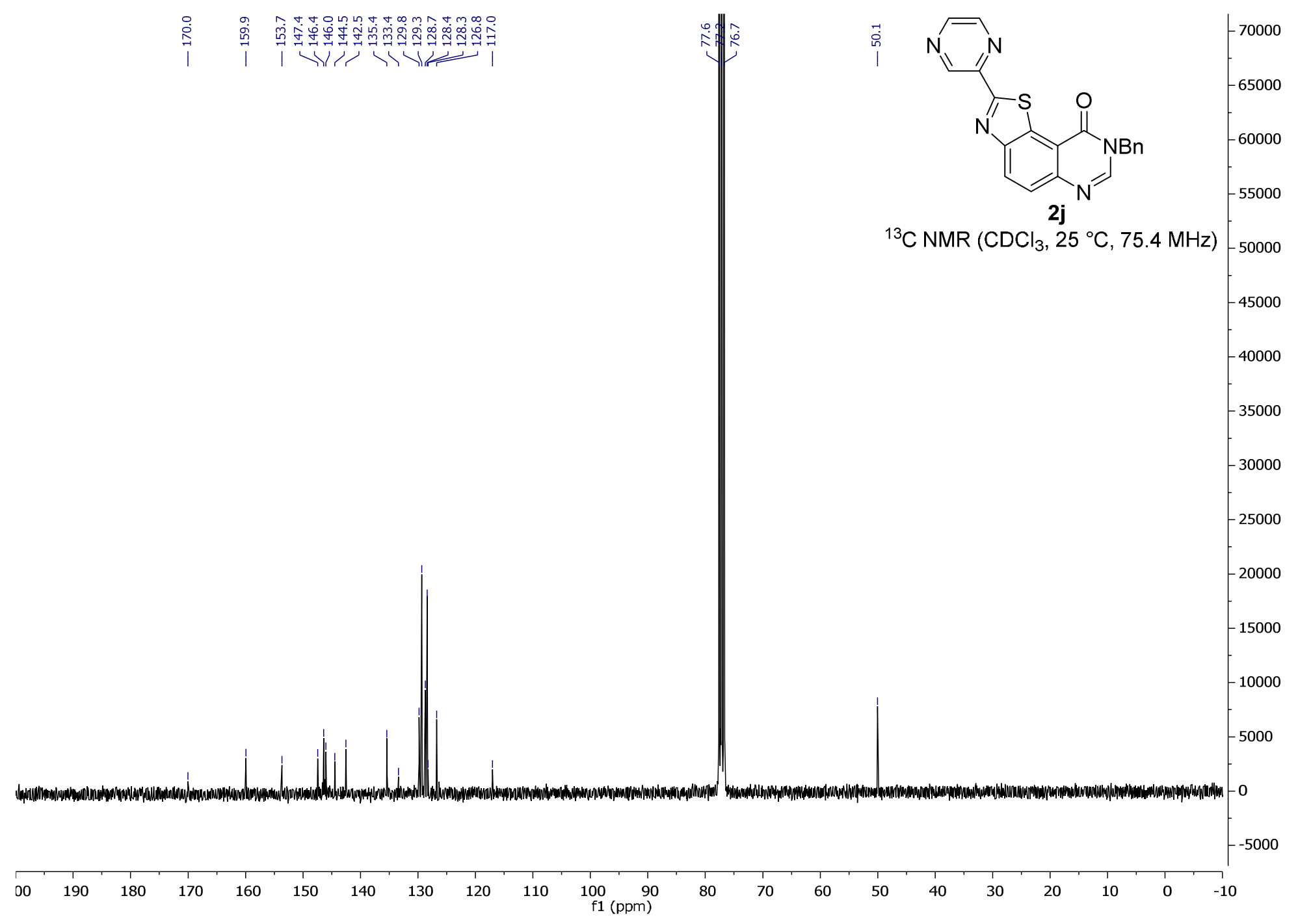


(E)- $N^{8}$-benzyl-2-styrylthiazolo[5,4-f]quinazolin-9(8H)-one (2k)

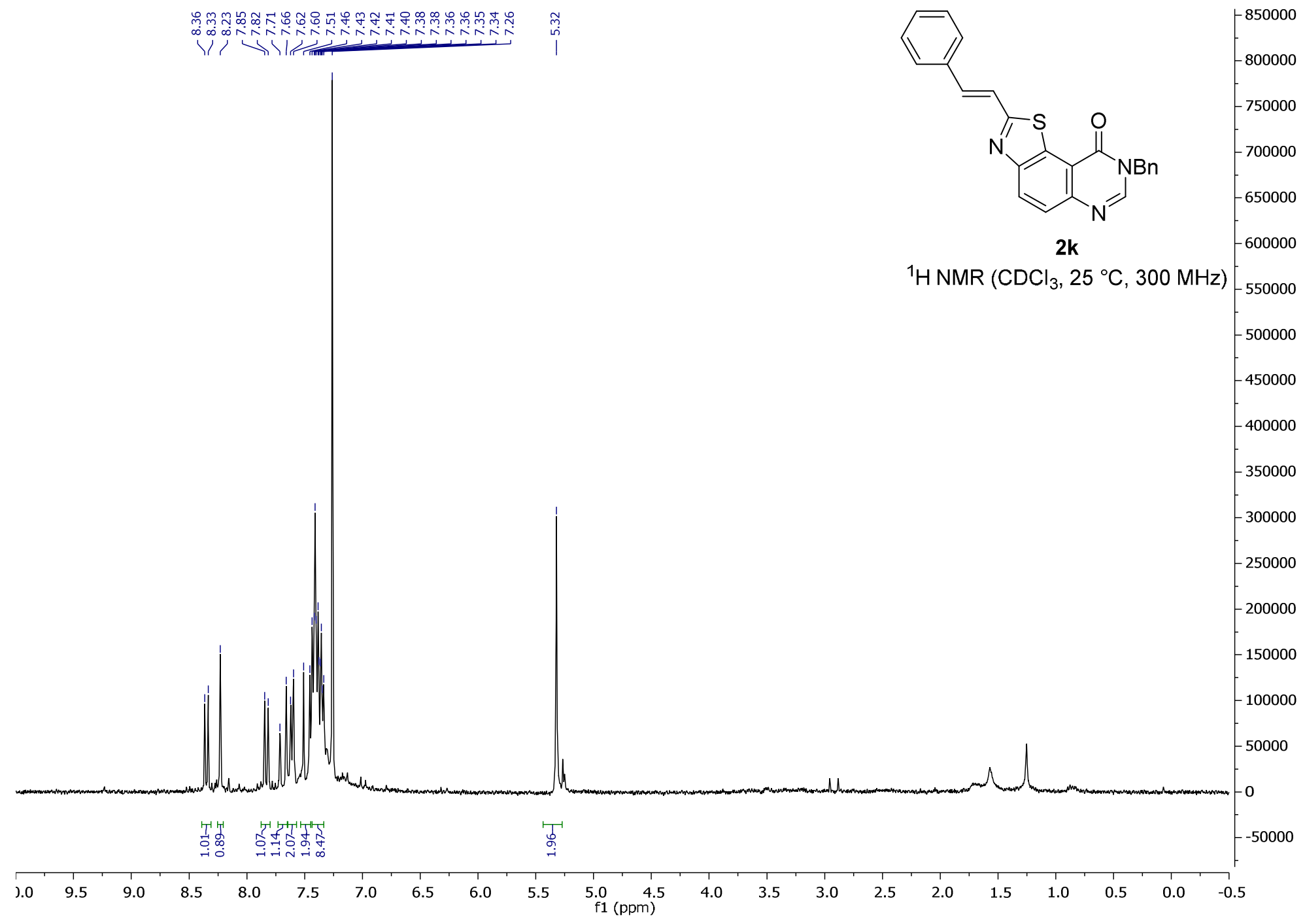




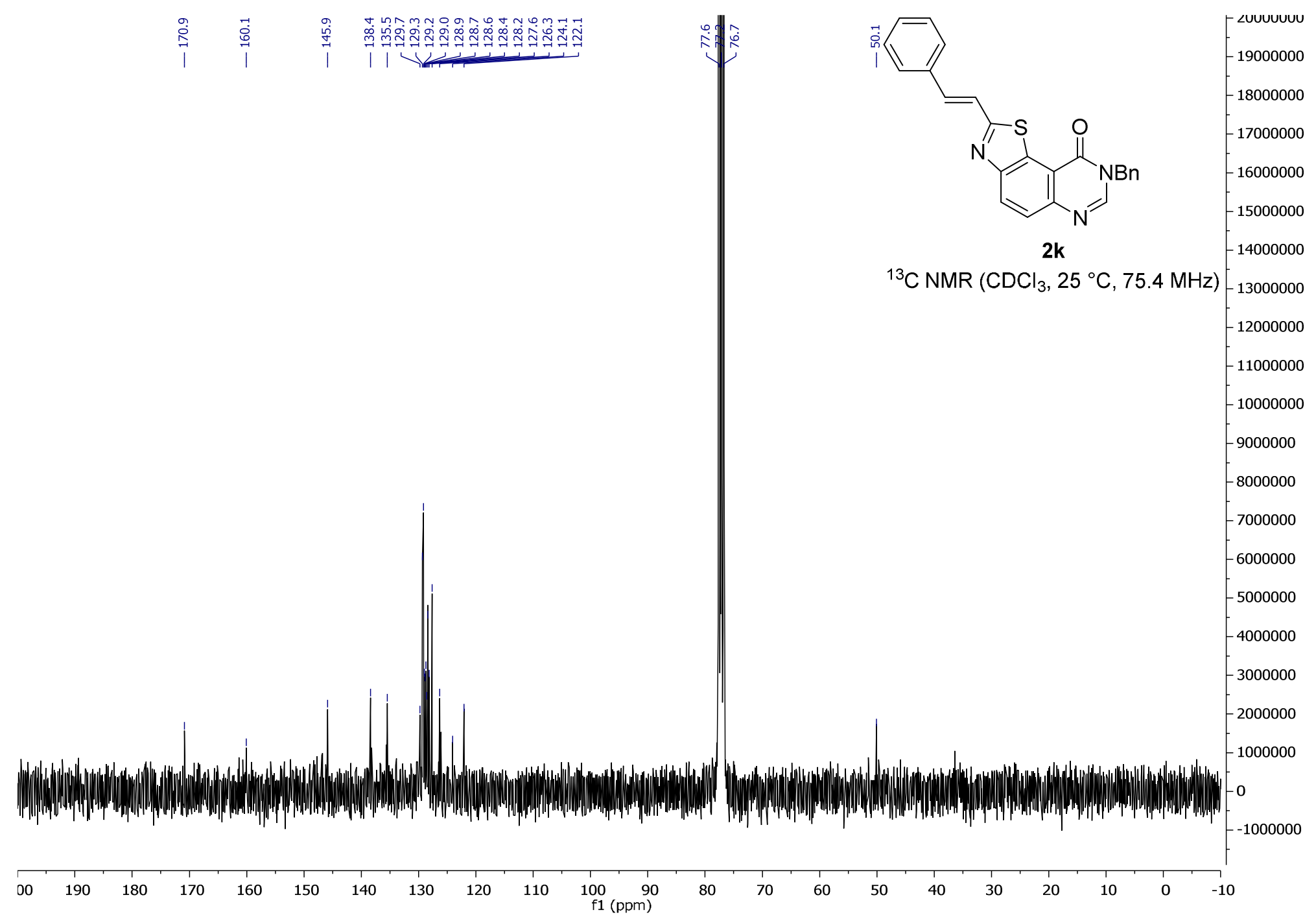


$N^{8}$-benzyl-2,7-diphenylthiazolo[5,4-f]quinazolin-9(8H)-one (3a)

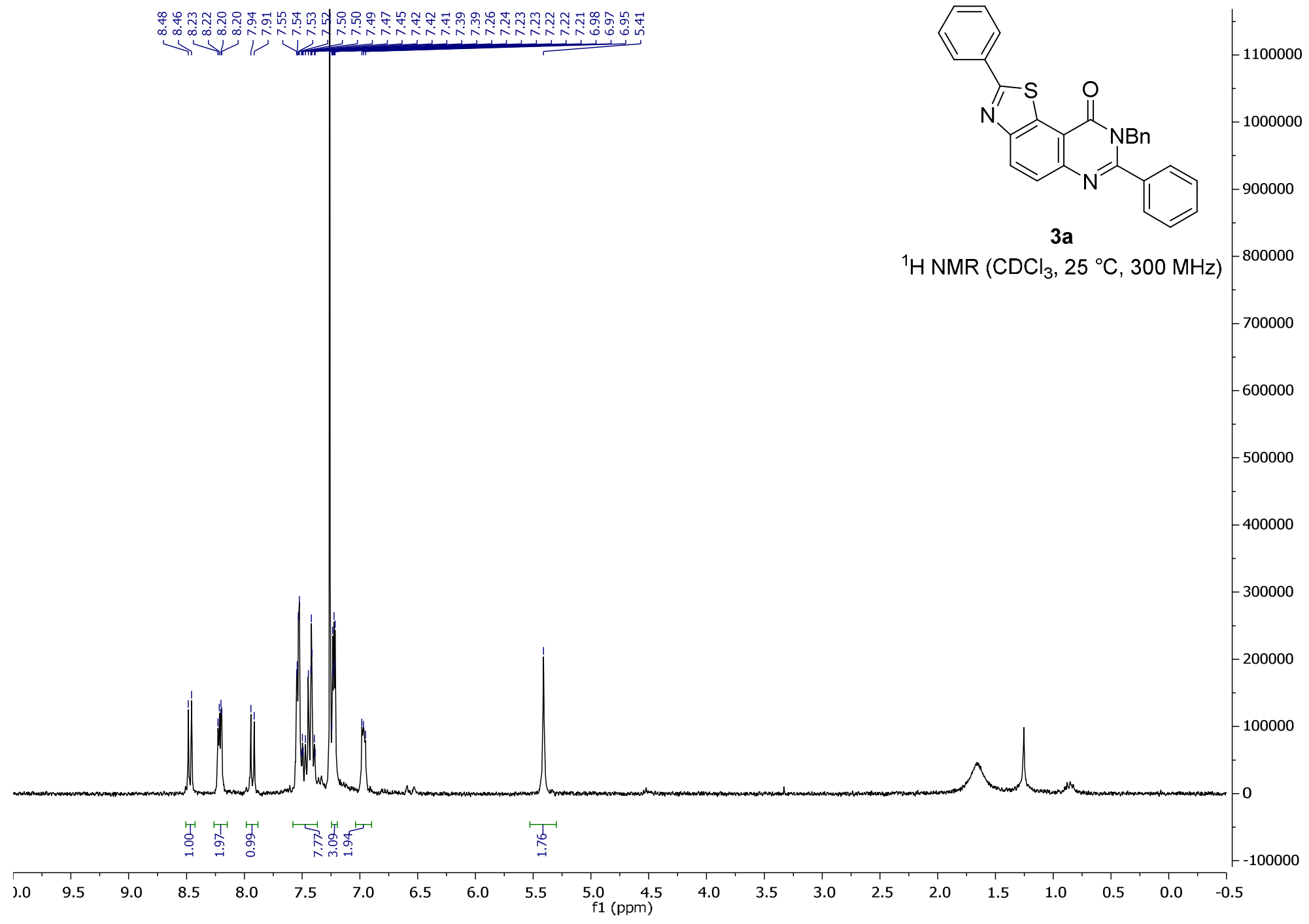




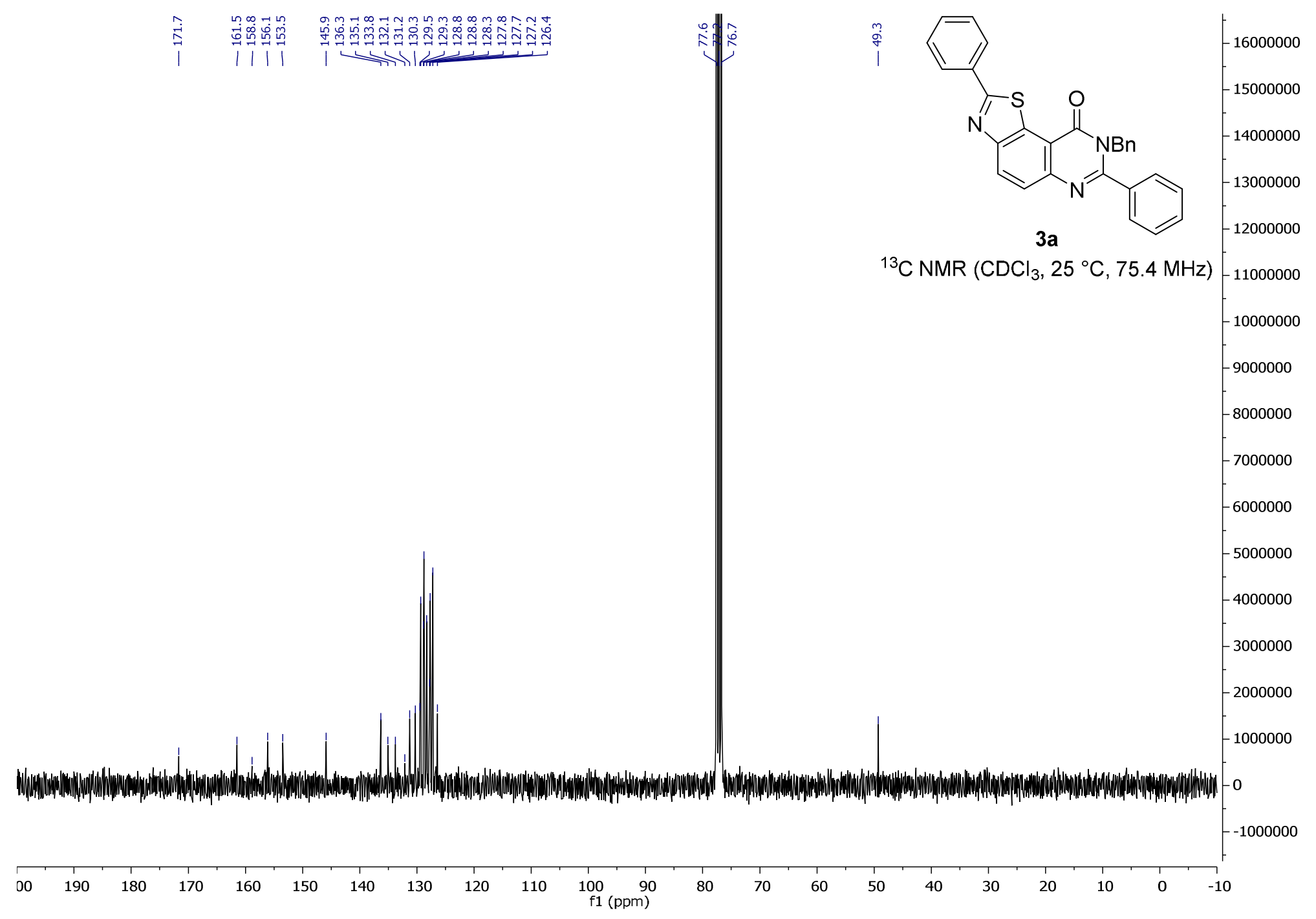


$N^{8}$-benzyl-2,7-di-p-tolylthiazolo[5,4-f]quinazolin-9(8H)-one (3b)

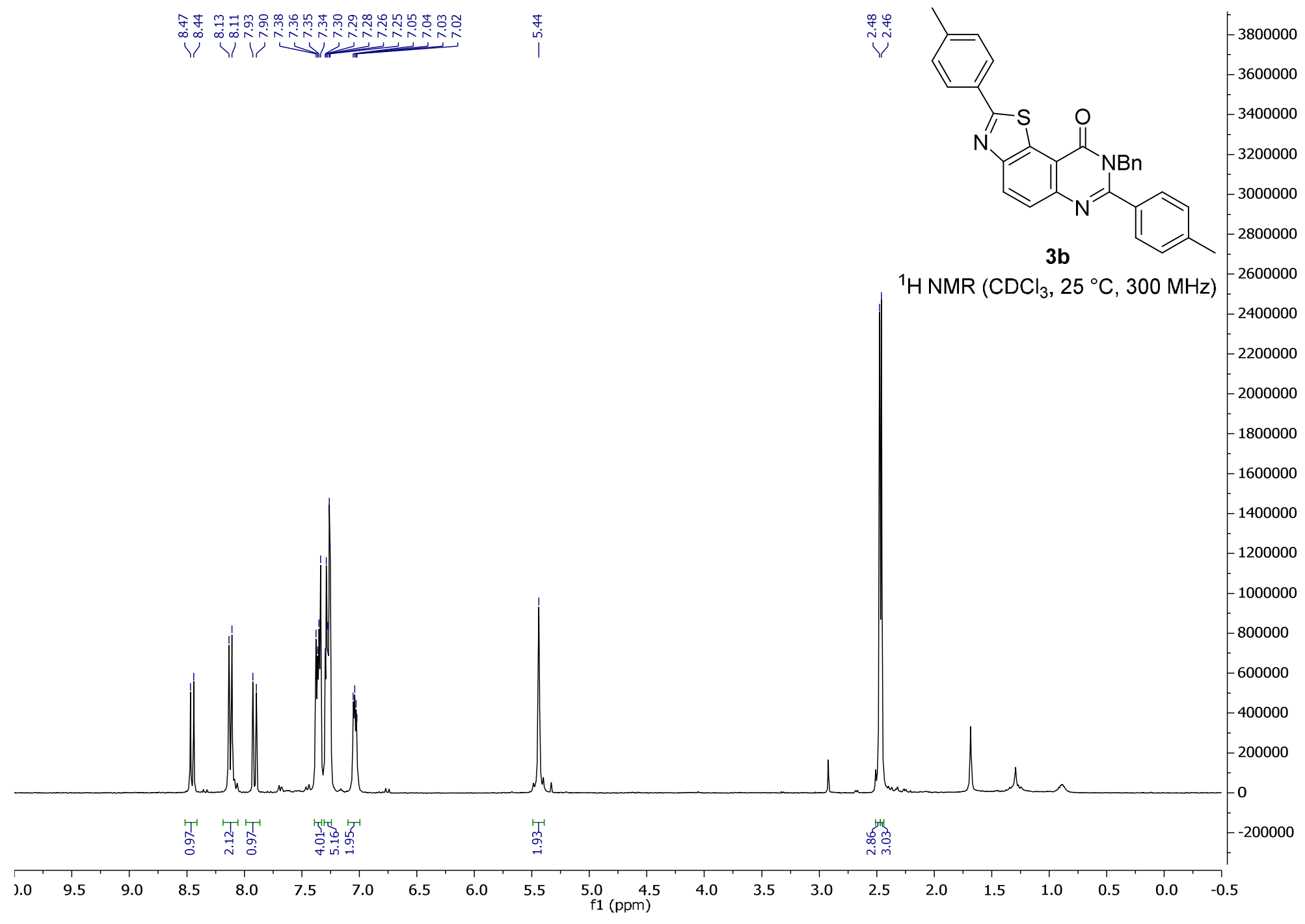




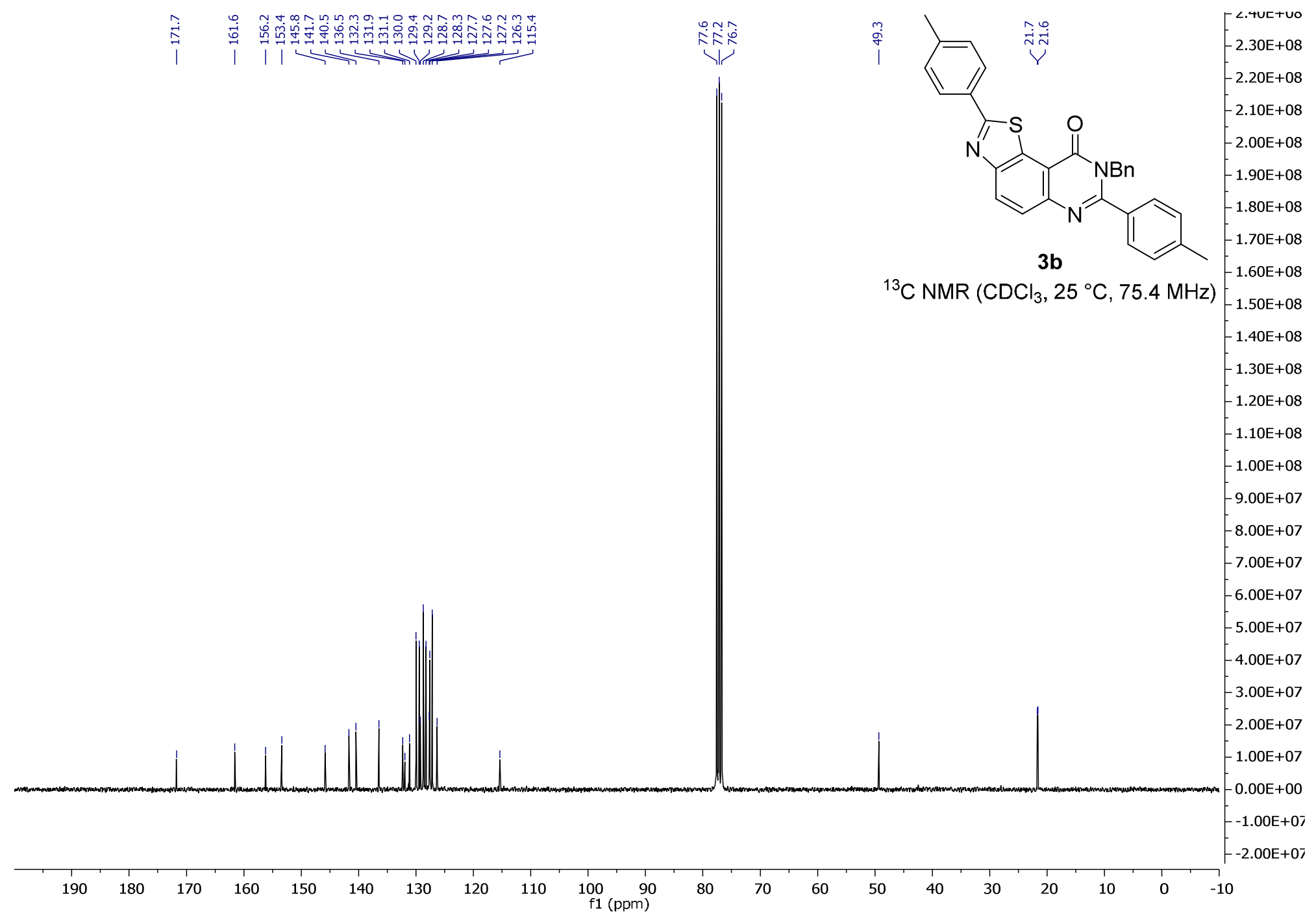


$N^{8}$-benzyl-2,7-bis(4-methoxyphenyl)thiazolo[5,4-f]quinazolin-9(8H)-one (3c)

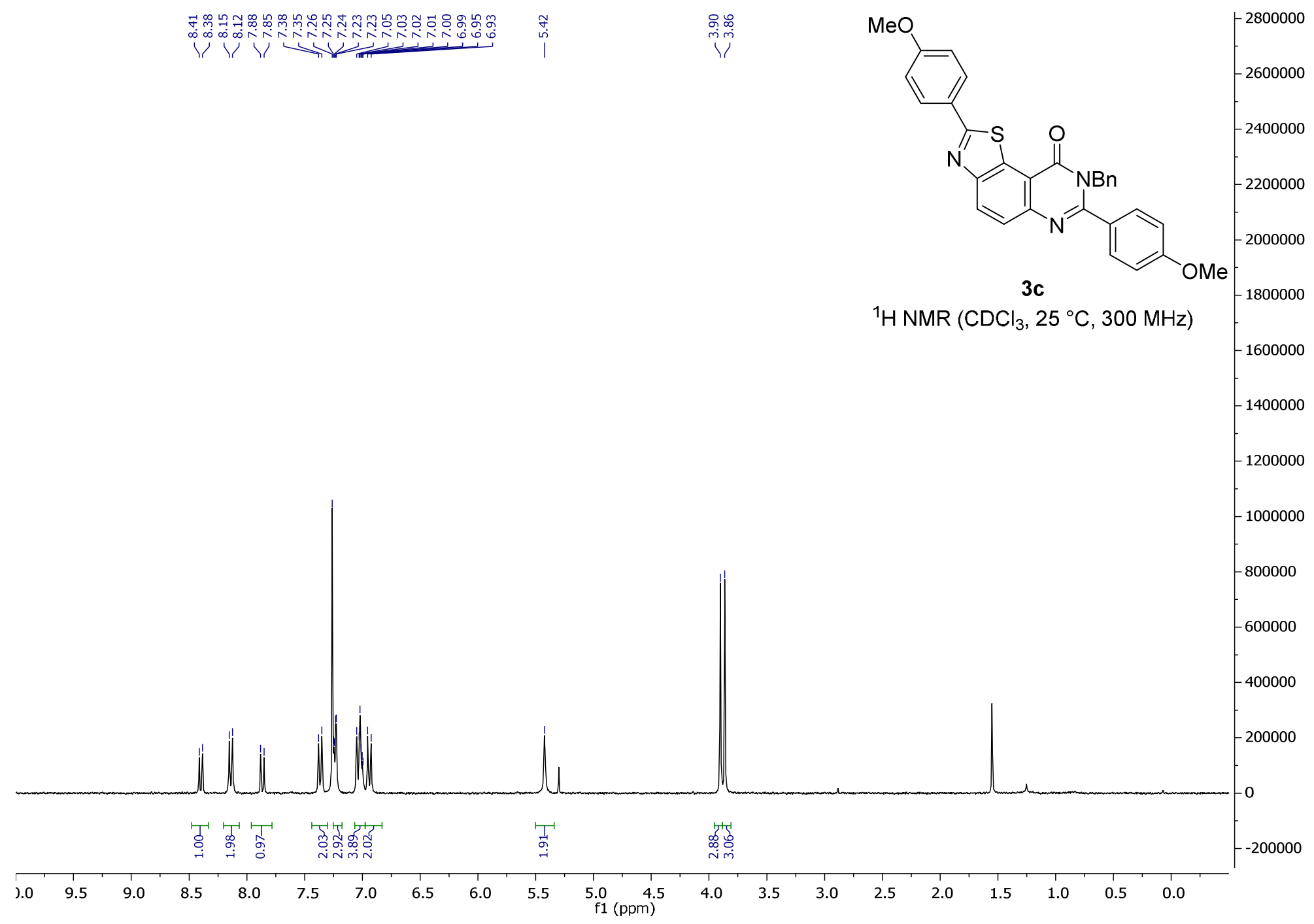




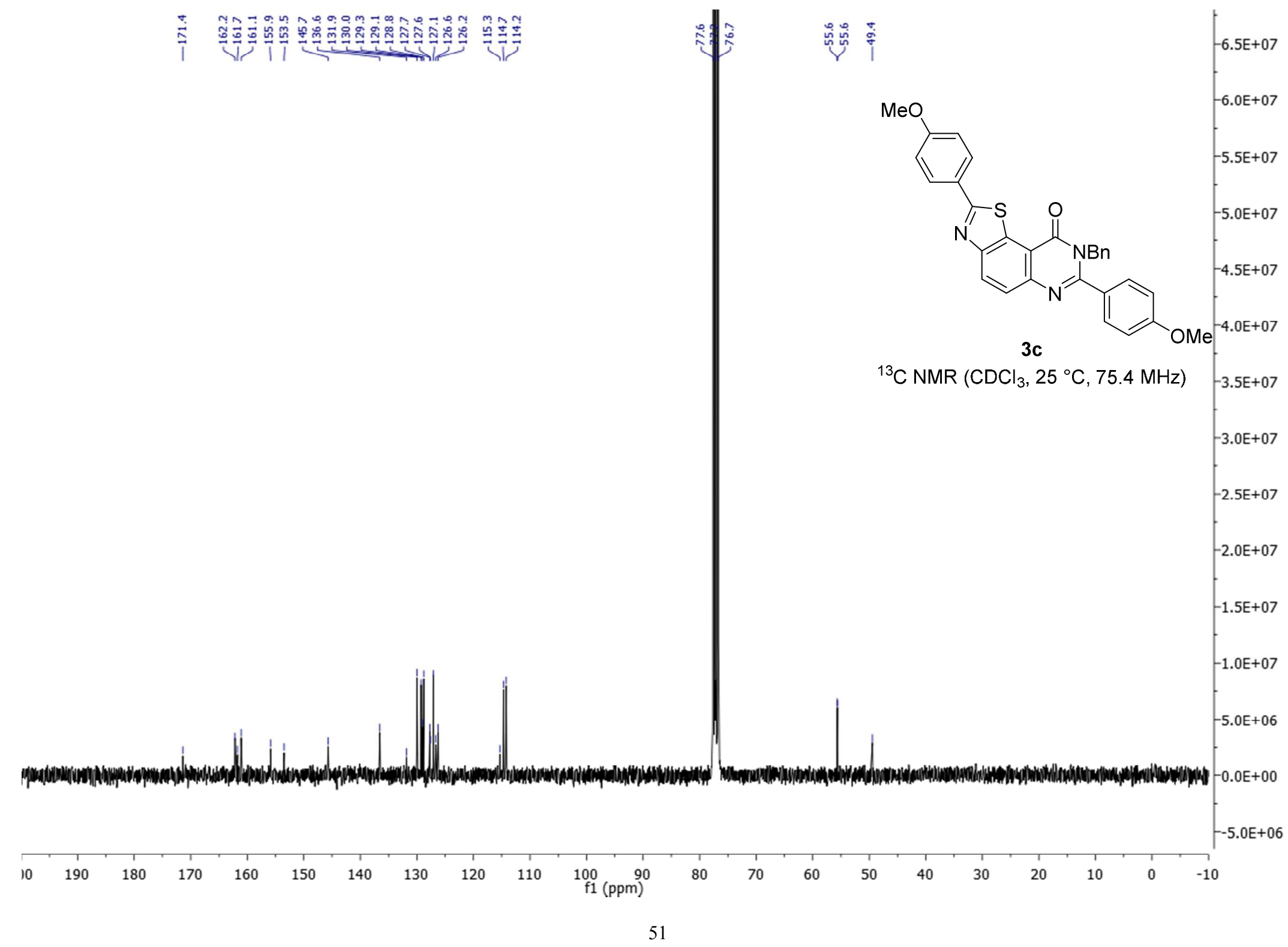


$N^{8}$-benzyl-2,7-bis(4-chlorophenyl)thiazolo[5,4-f]quinazolin-9(8H)-one (3d)

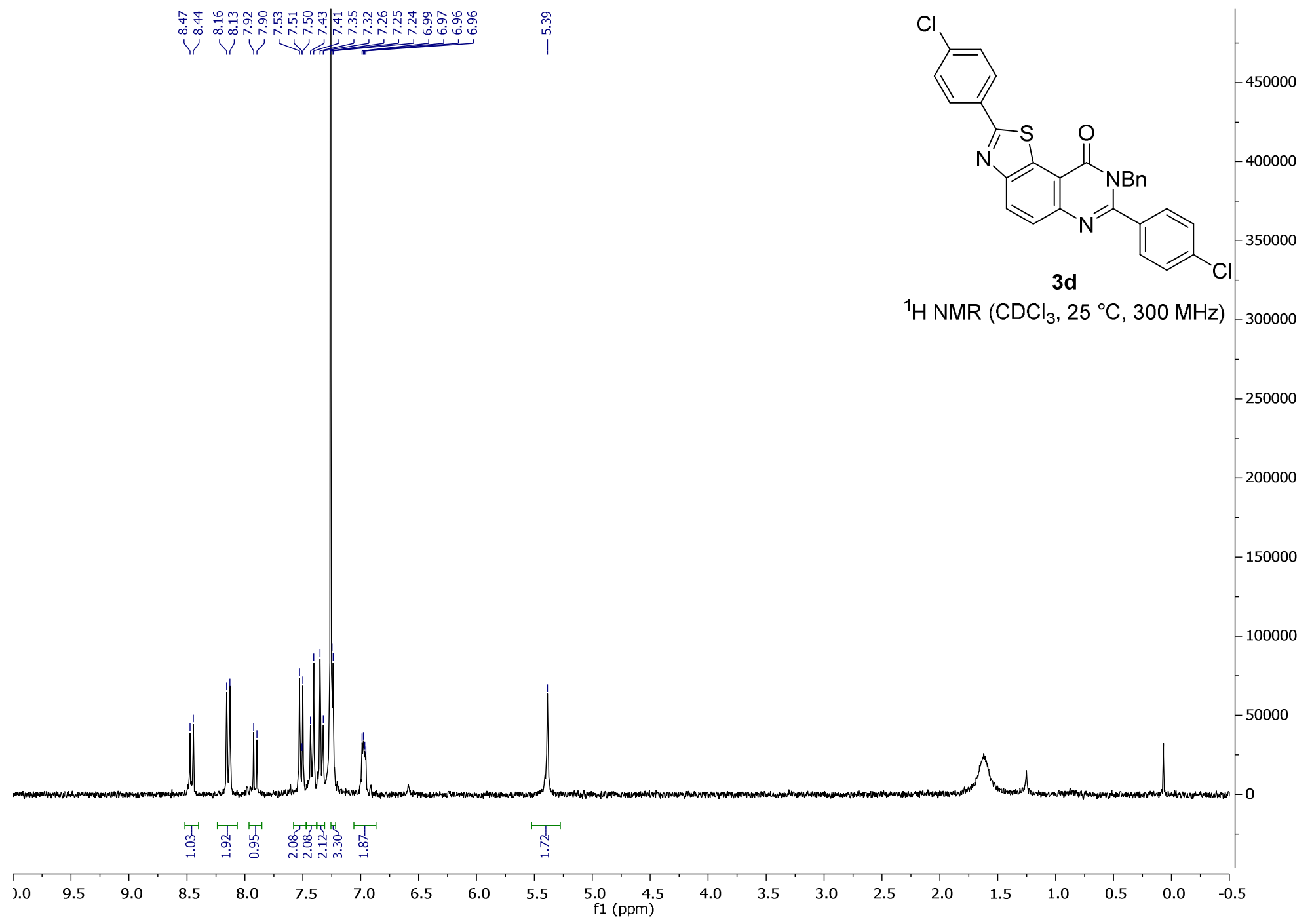




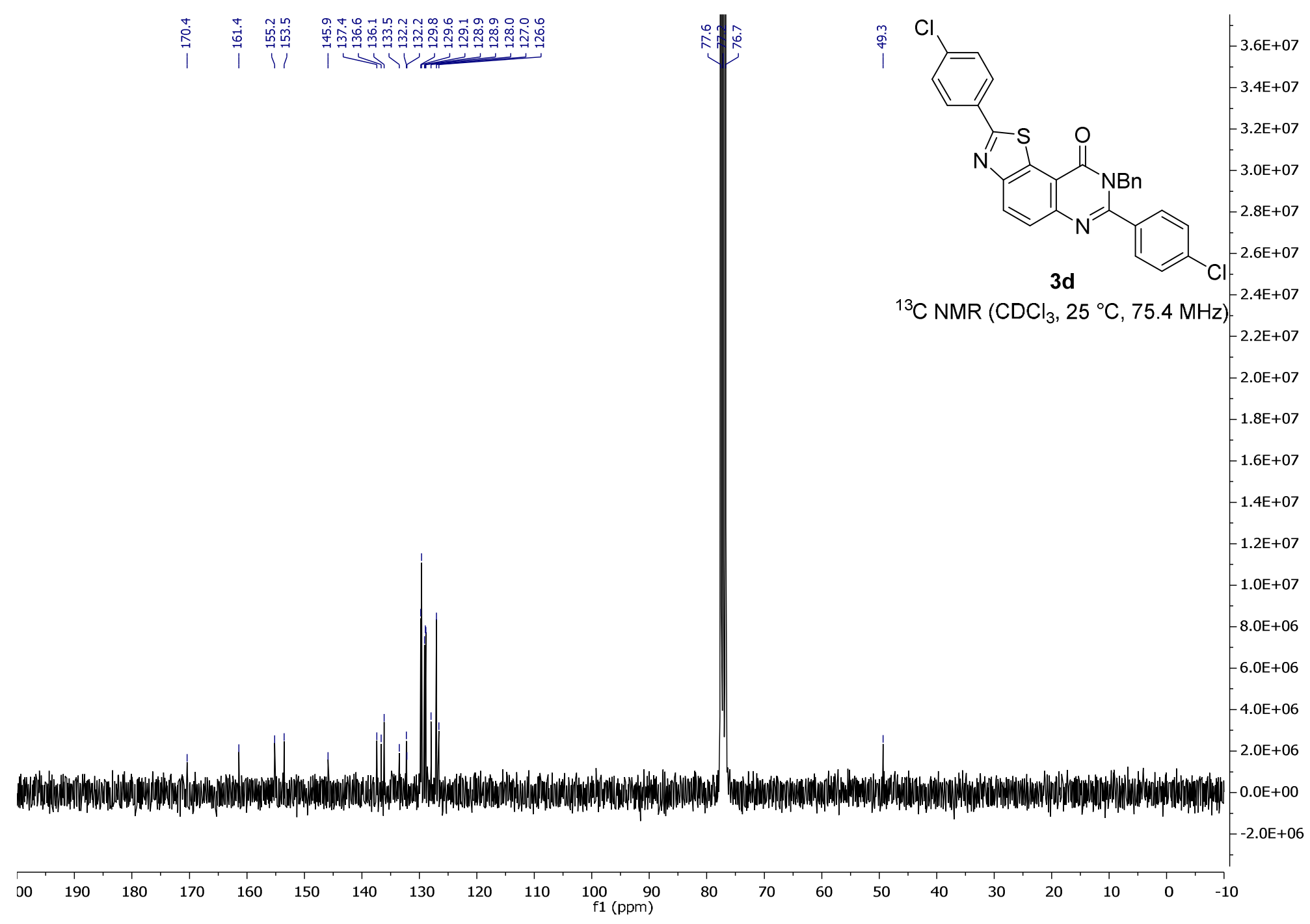


$N^{8}$-benzyl-2,7-bis(4-fluorophenyl)thiazolo[5,4-f]quinazolin-9(8H)-one (3e)

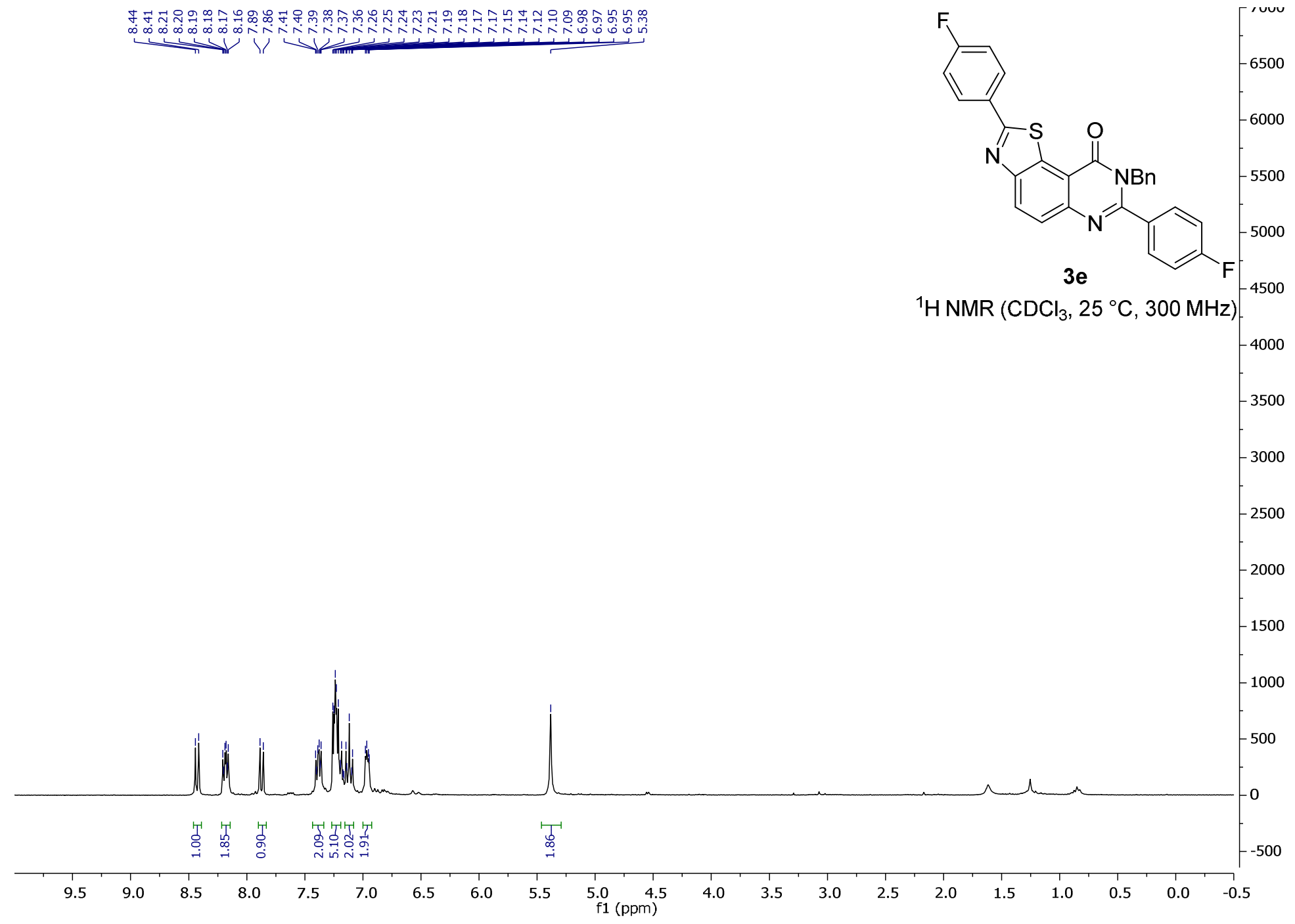




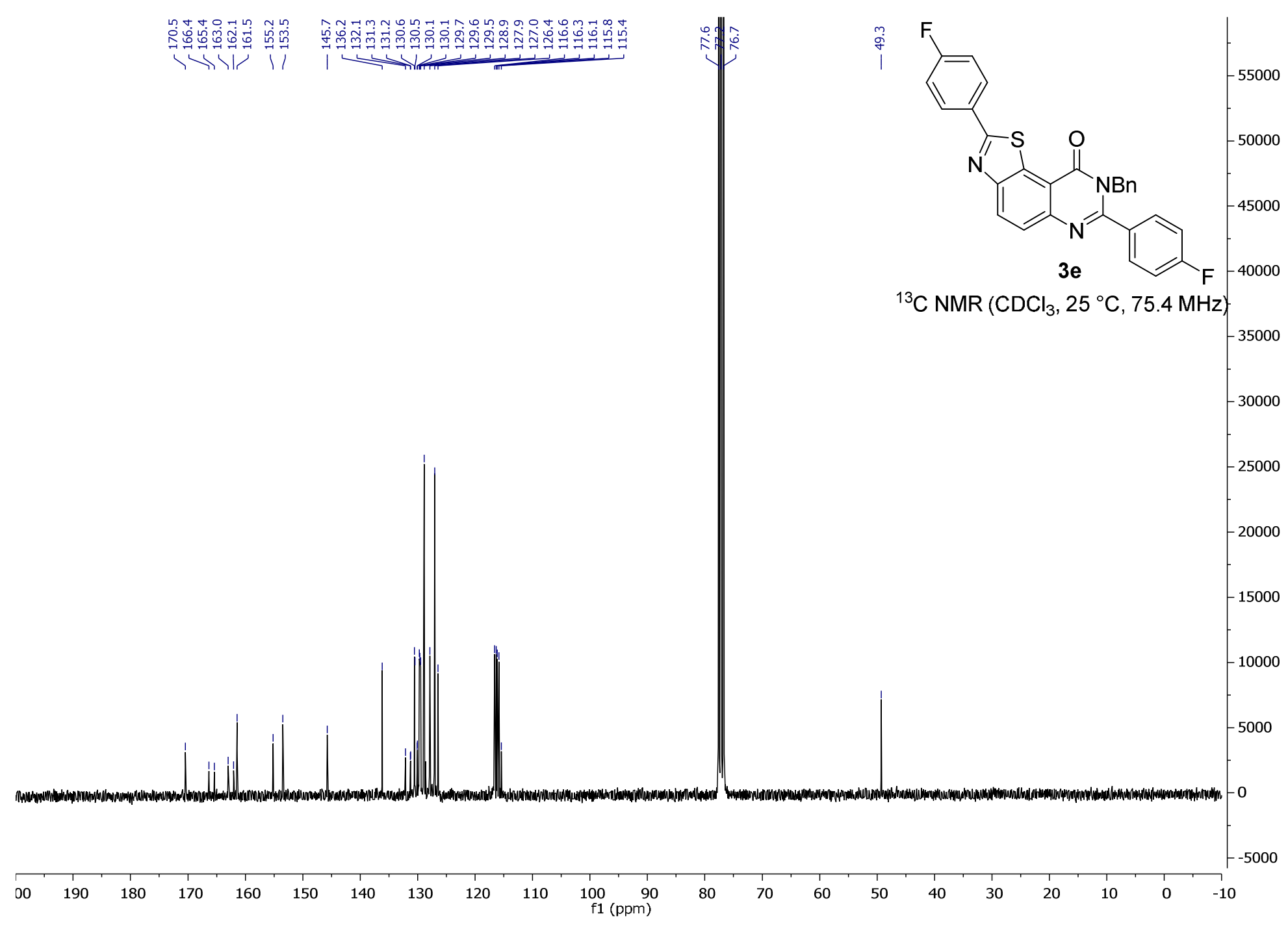


4,4'-(N-benzyl-9-oxo-8,9-dihydrothiazolo[5,4-f]quinazoline-2,7-diyl)dibenzonitrile (3f)

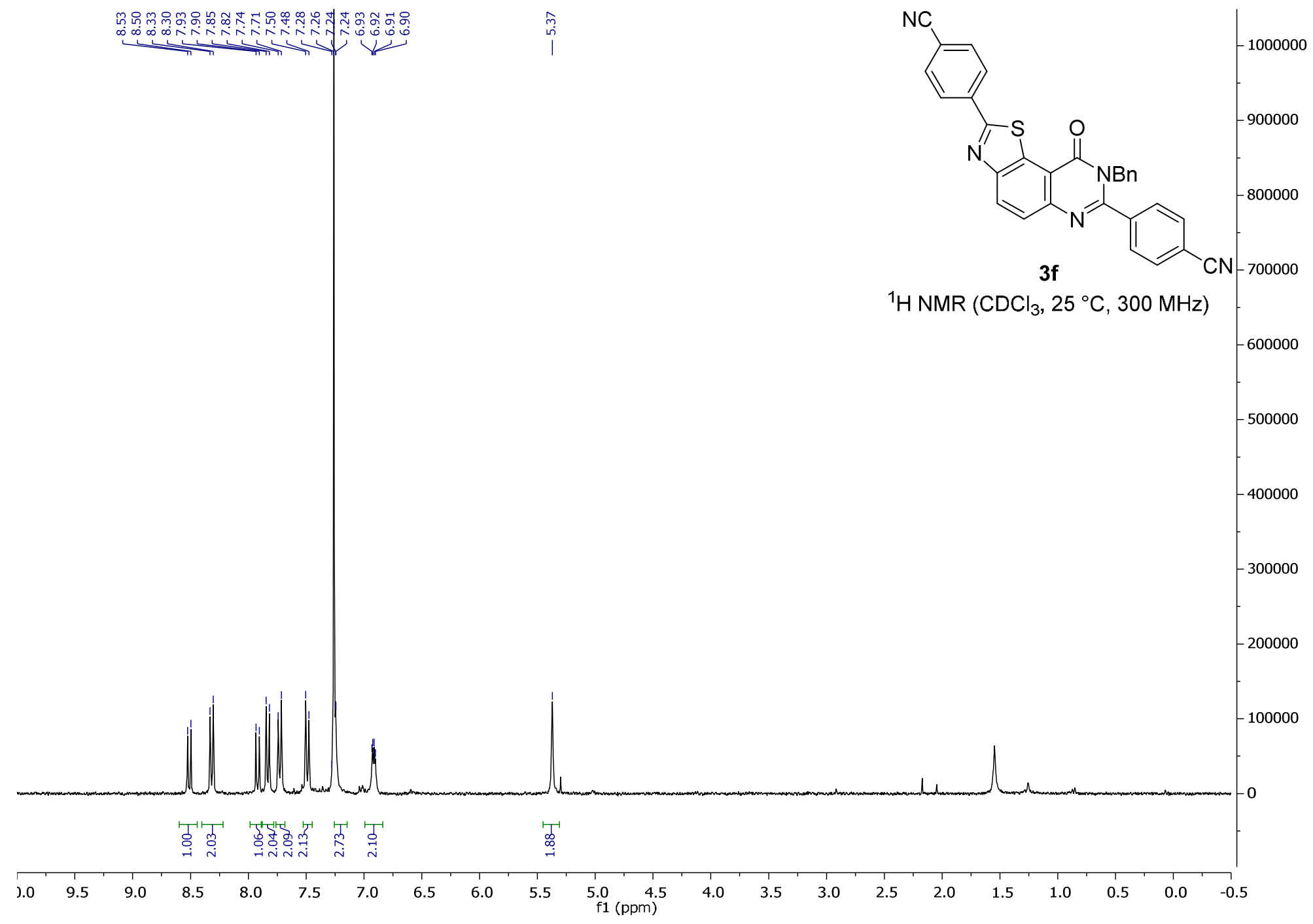




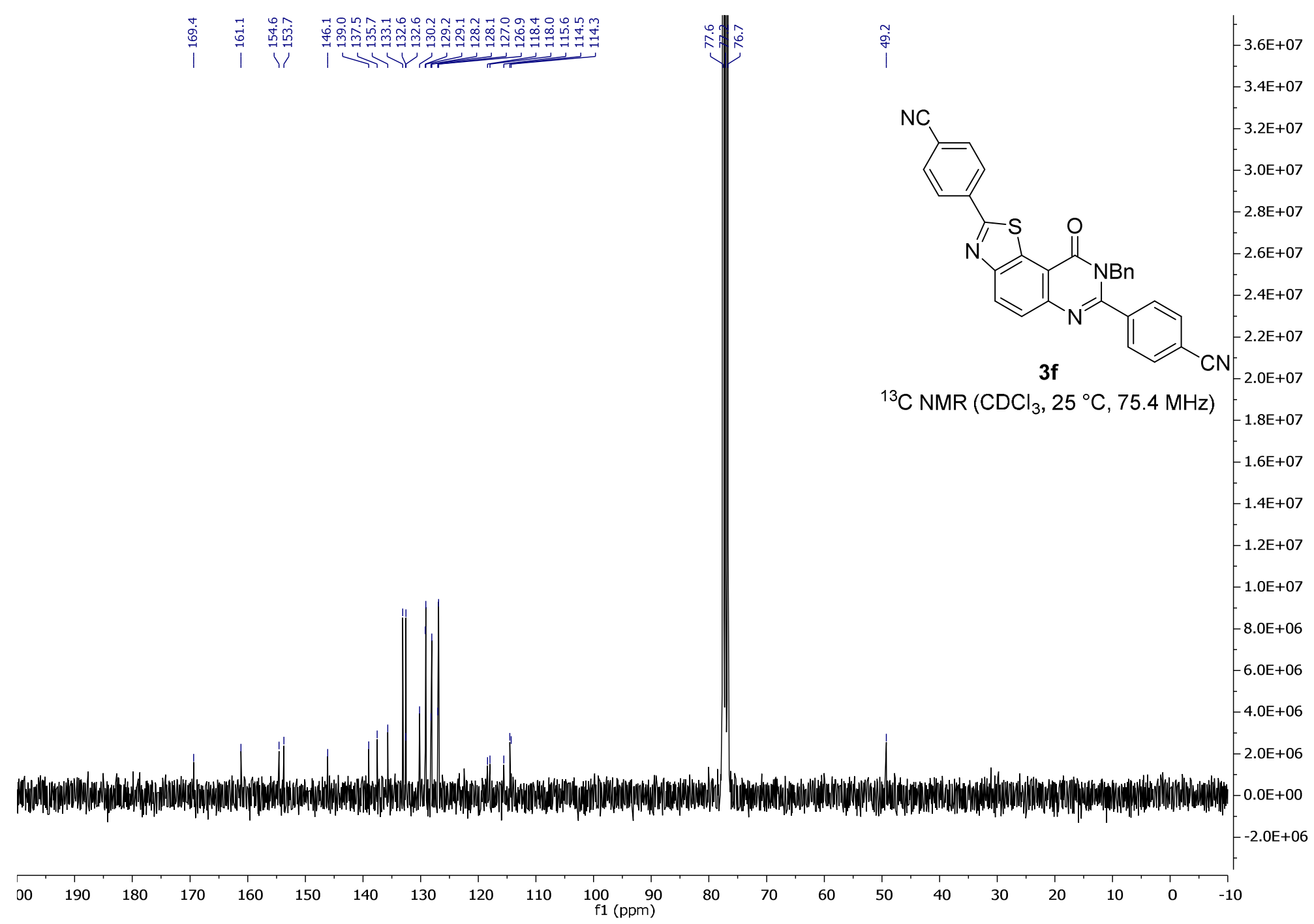


$N^{8}$-benzyl-2-phenyl-7-(p-tolyl)thiazolo[5,4-f]quinazolin-9(8H)-one (4a)

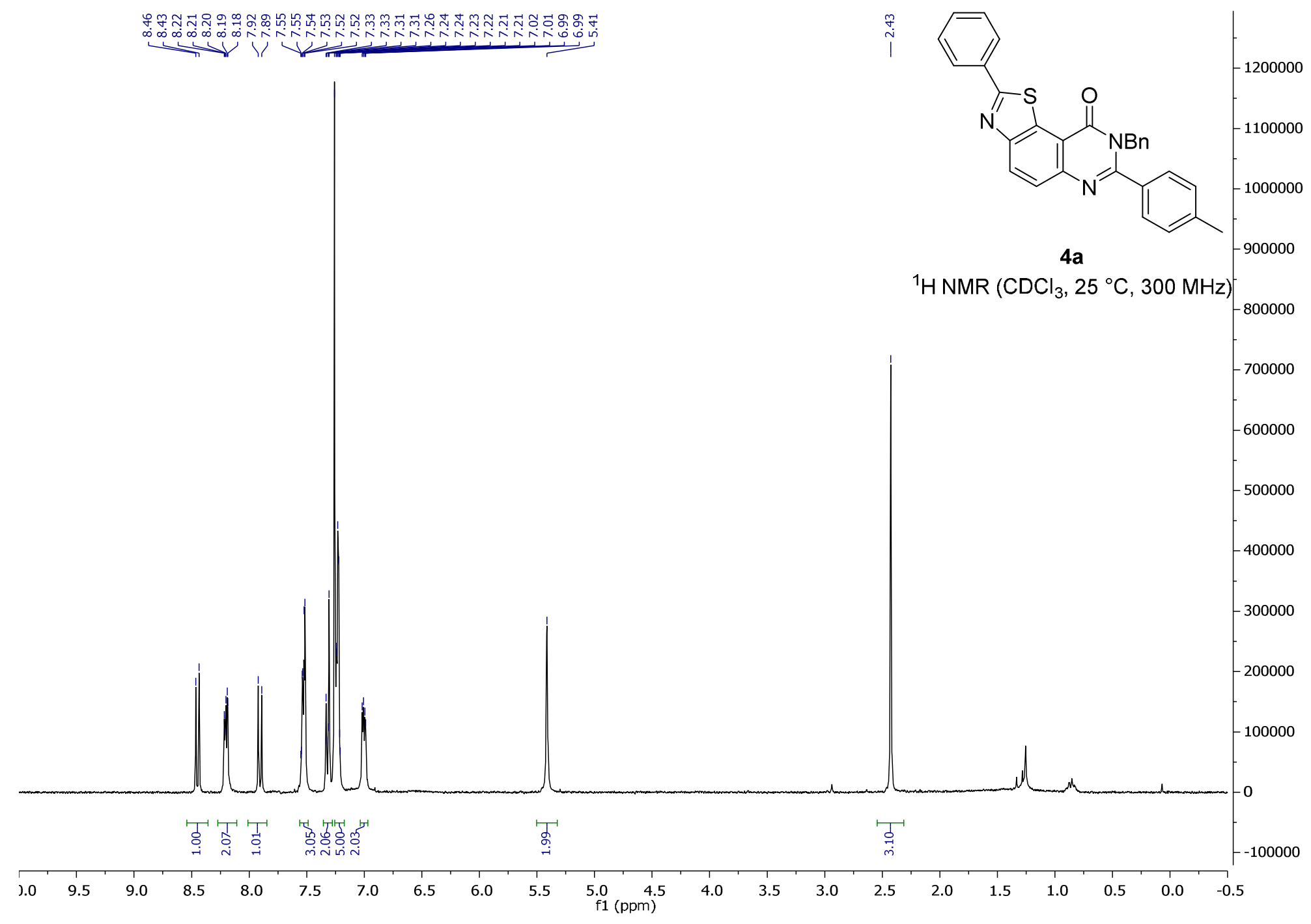




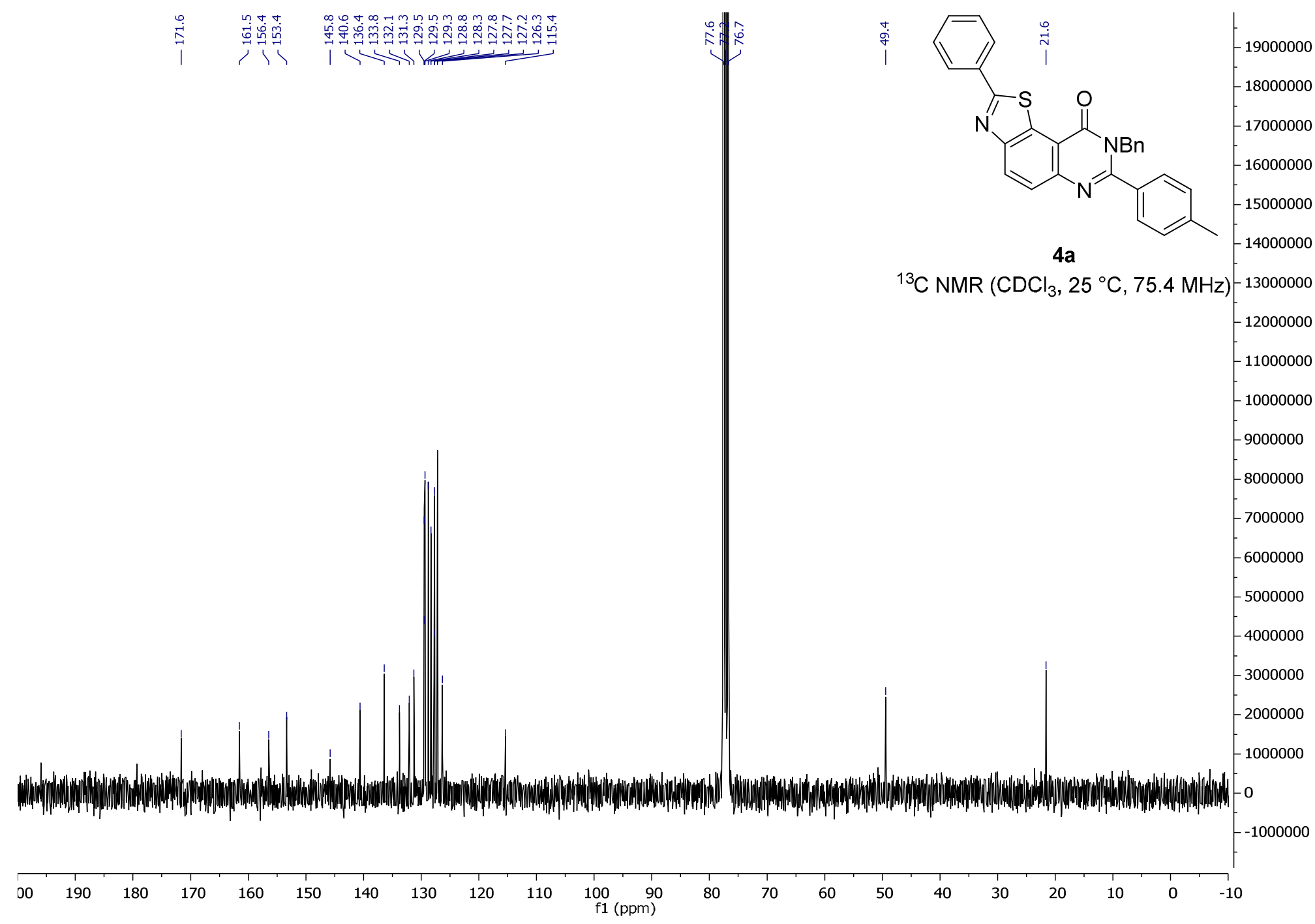


4-( $N^{8}$-benzyl-9-oxo-2-( $p$-tolyl)-8,9-dihydrothiazolo[5,4-f]quinazolin-7-yl)benzonitrile (4b)

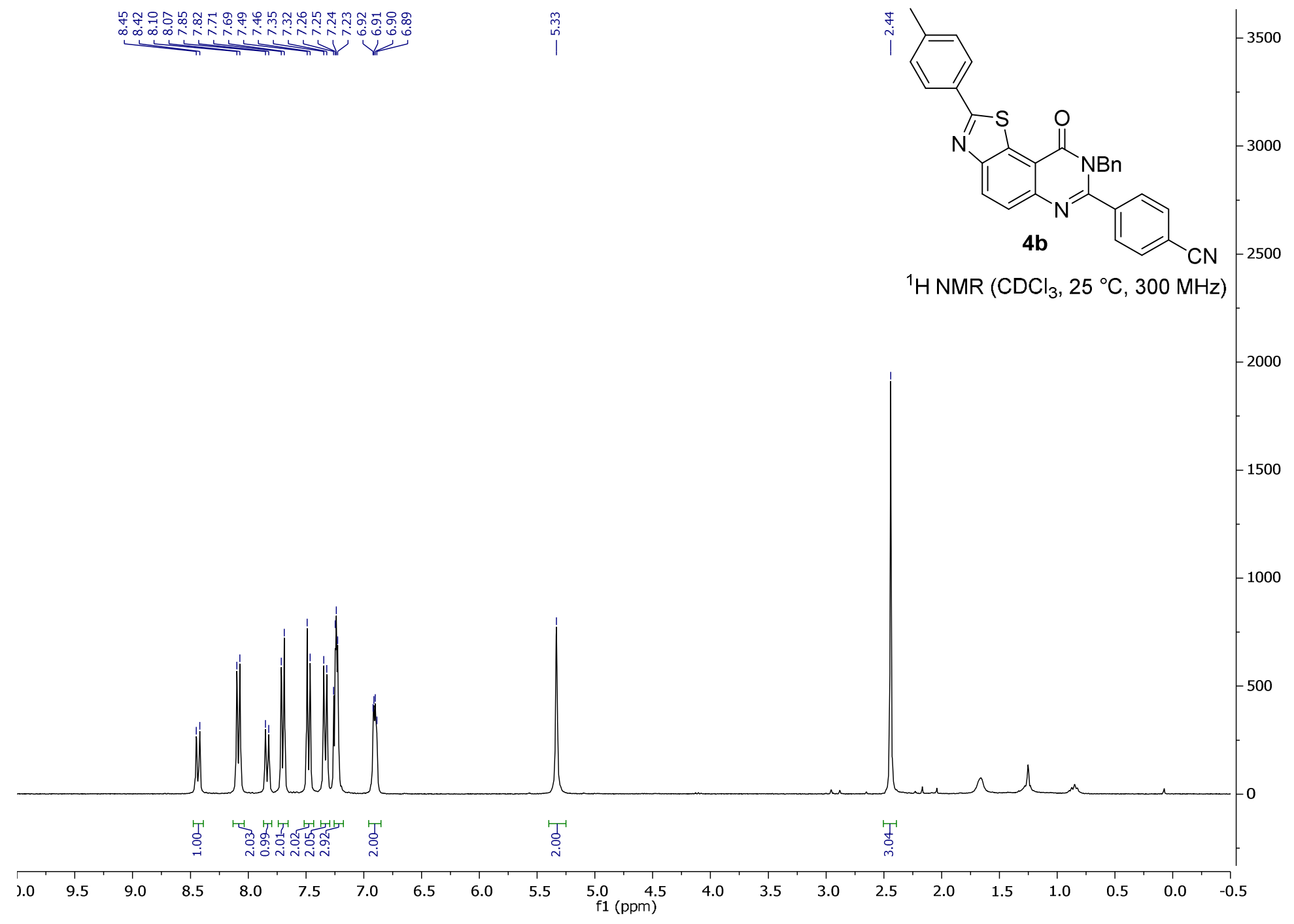




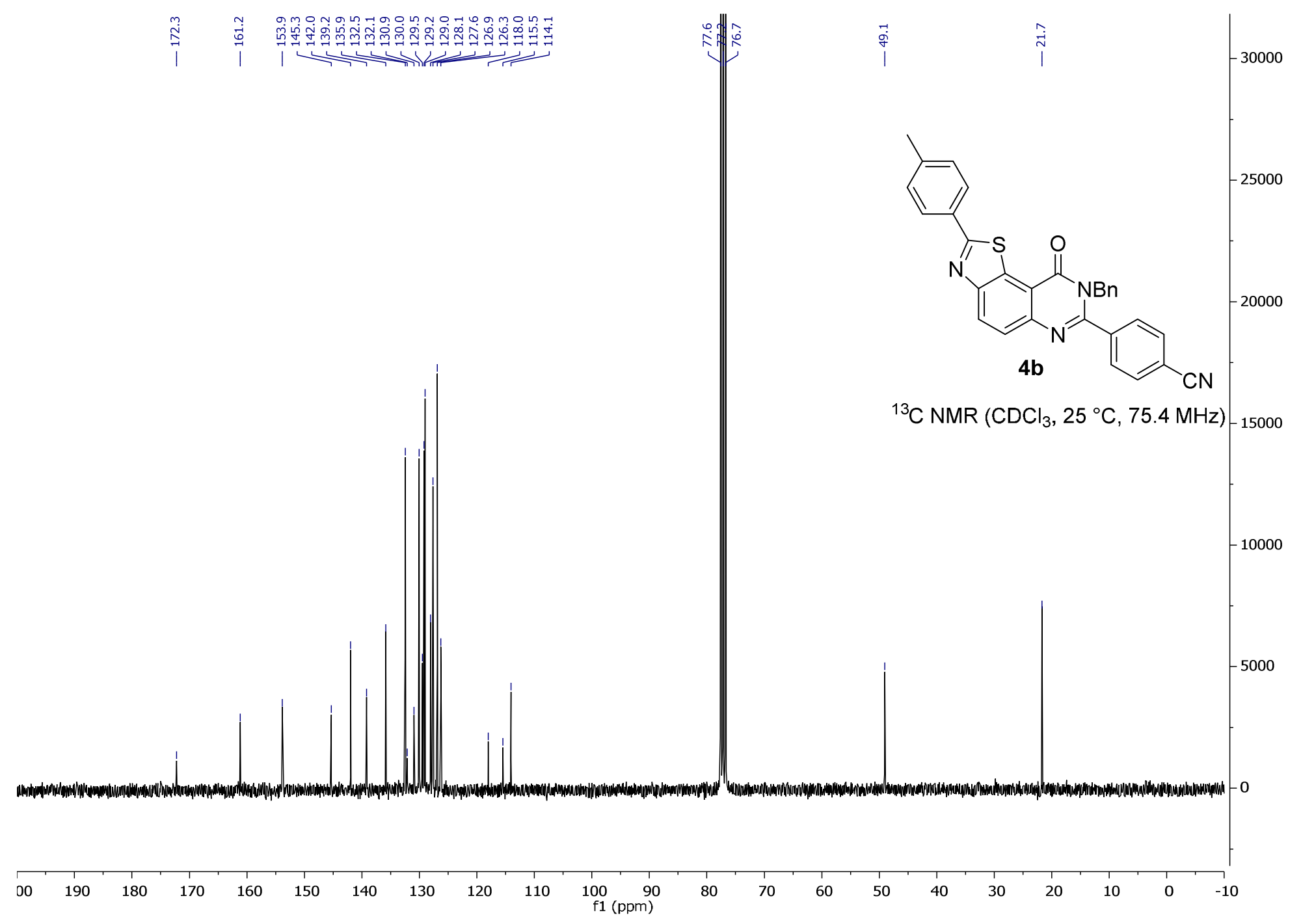


4-( $N^{8}$-benzyl-2-(4-methoxyphenyl)-9-oxo-8,9-dihydrothiazolo[5,4-f]quinazolin-7-yl)benzonitrile (4ca)

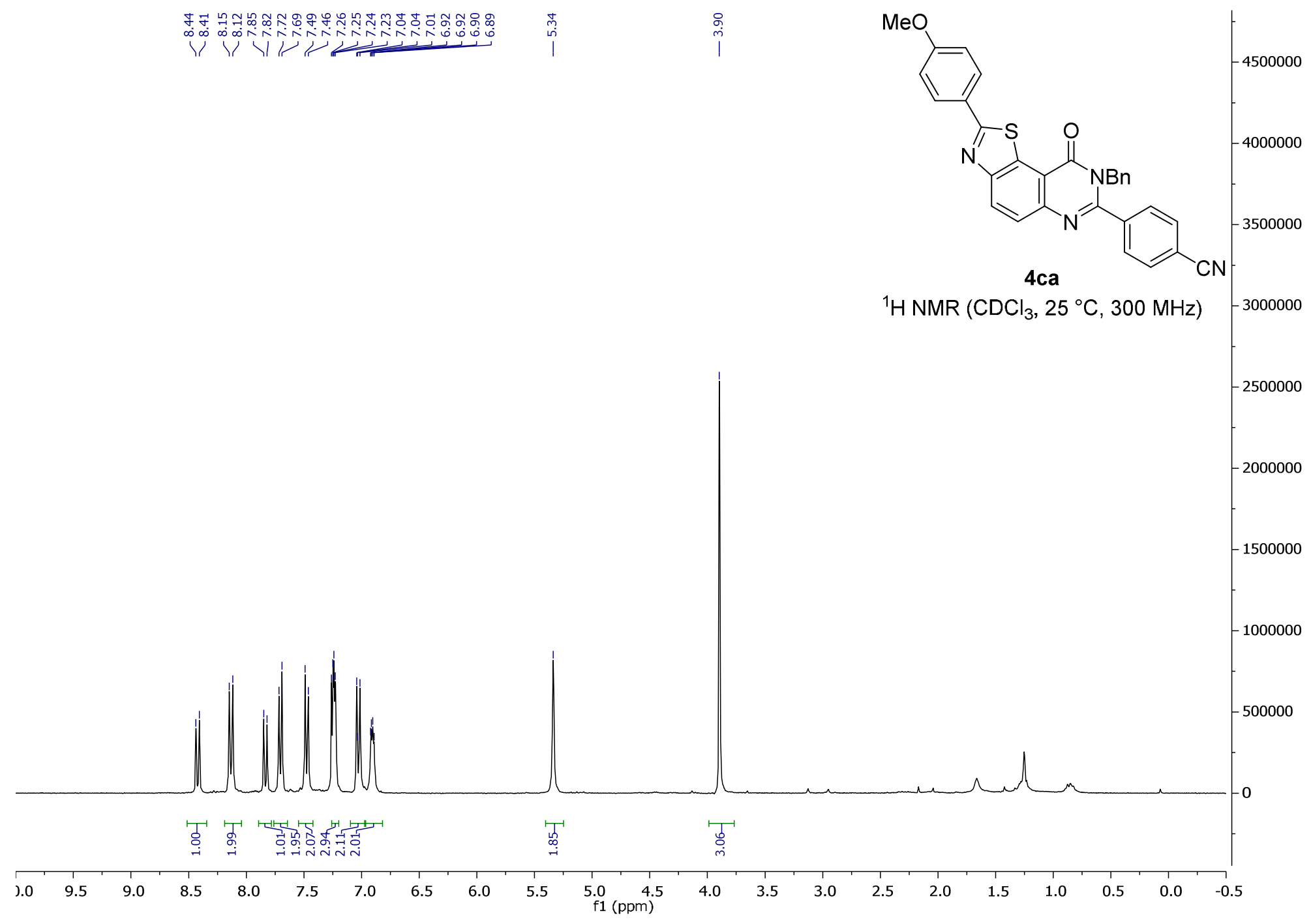




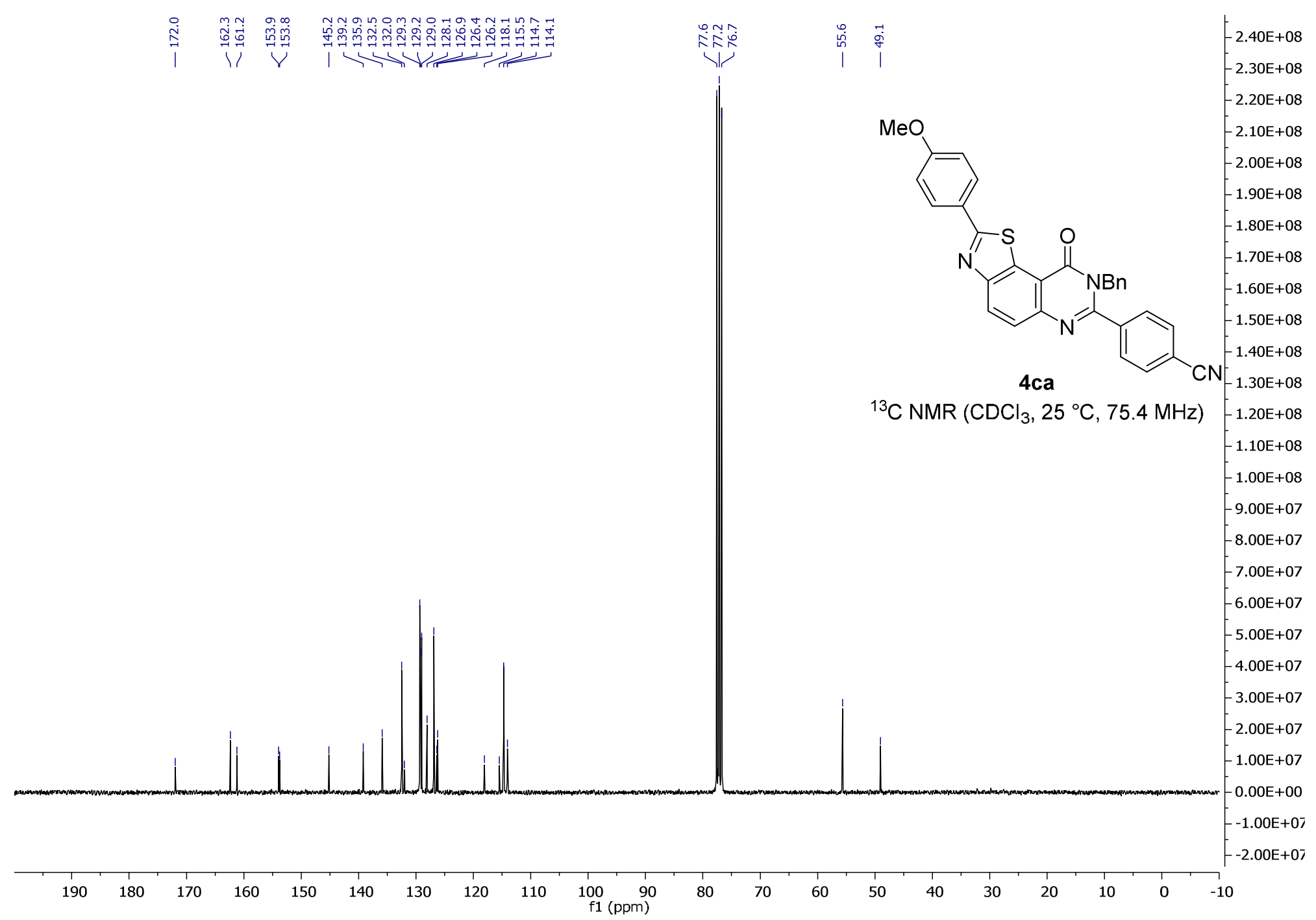


$N^{8}$-benzyl-7-(4-fluorophenyl)-2-(4-methoxyphenyl)thiazolo[5,4-f]quinazolin-9(8H)-one (4cb)

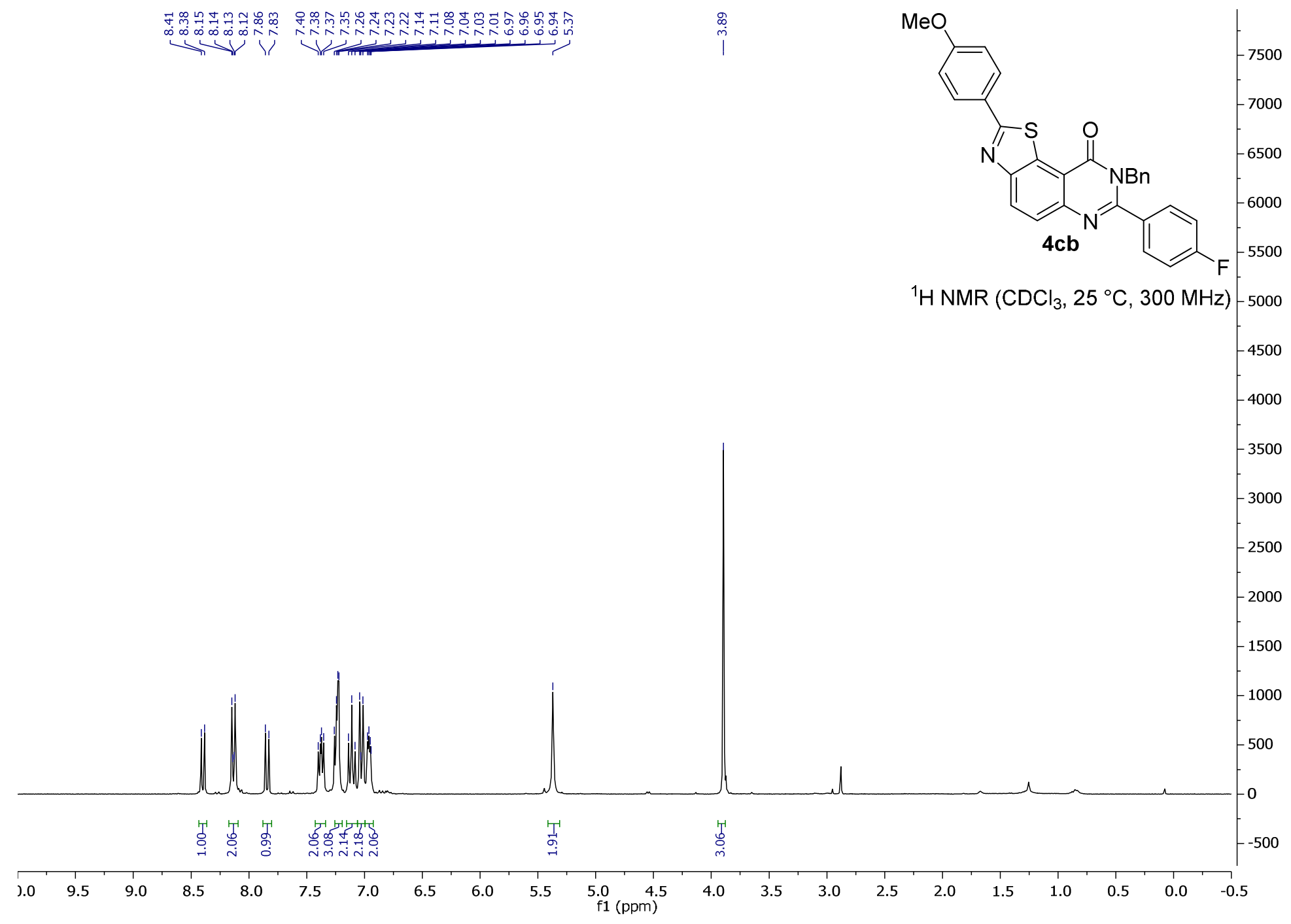




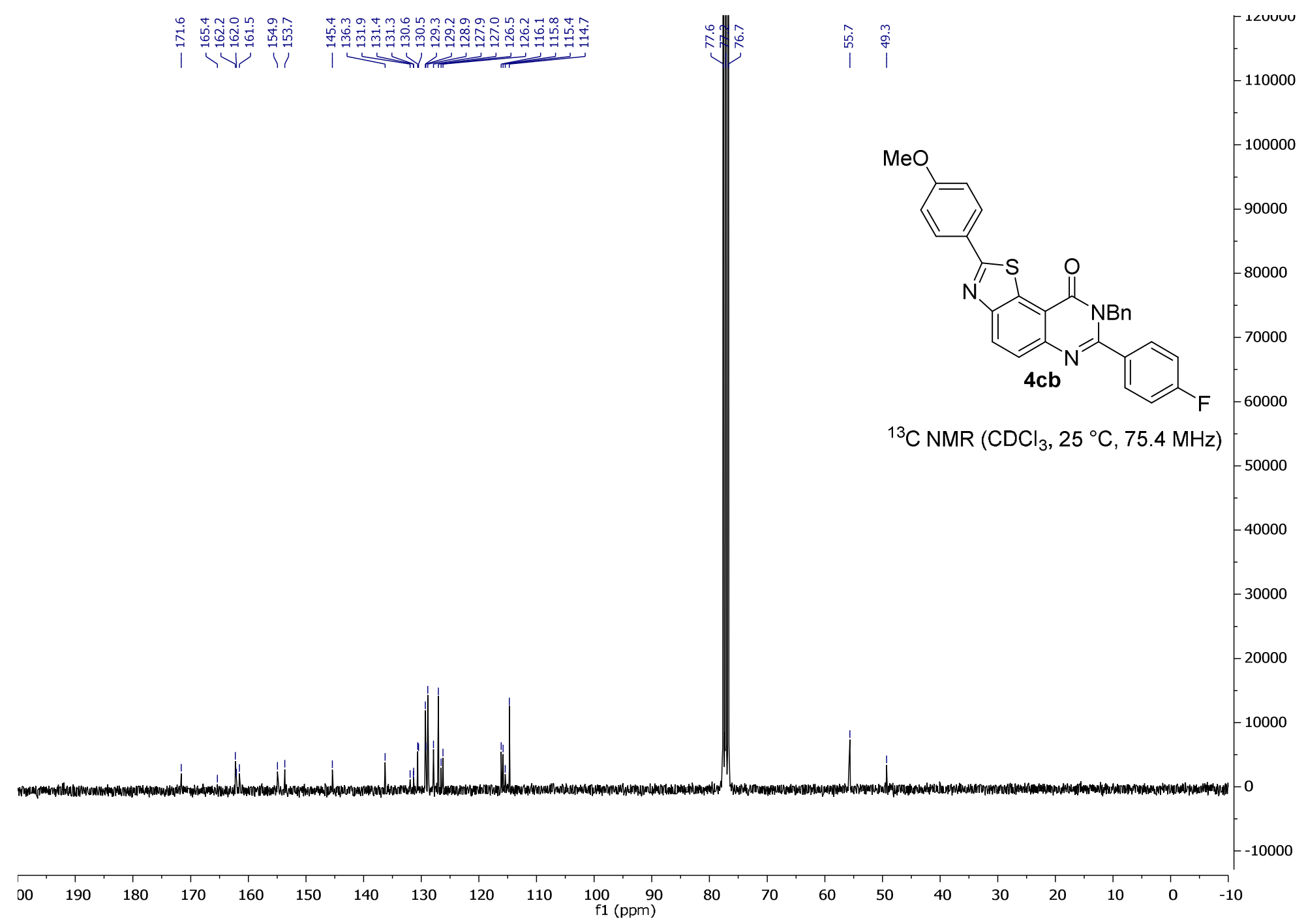


4-( $N^{8}$-benzyl-2-(4-chlorophenyl)-9-oxo-8,9-dihydrothiazolo[5,4-f] quinazolin-7-yl)benzonitrile (4da)

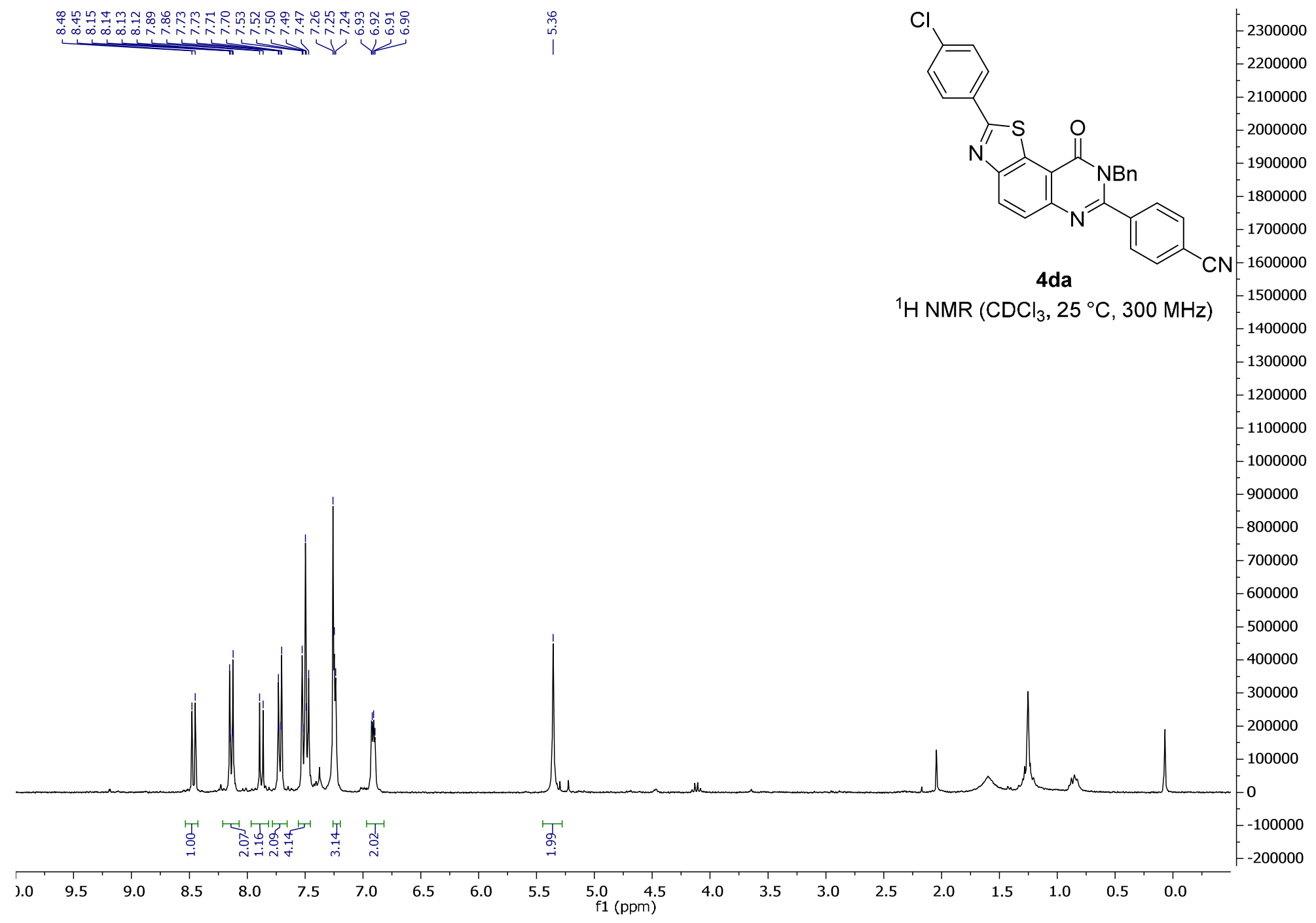




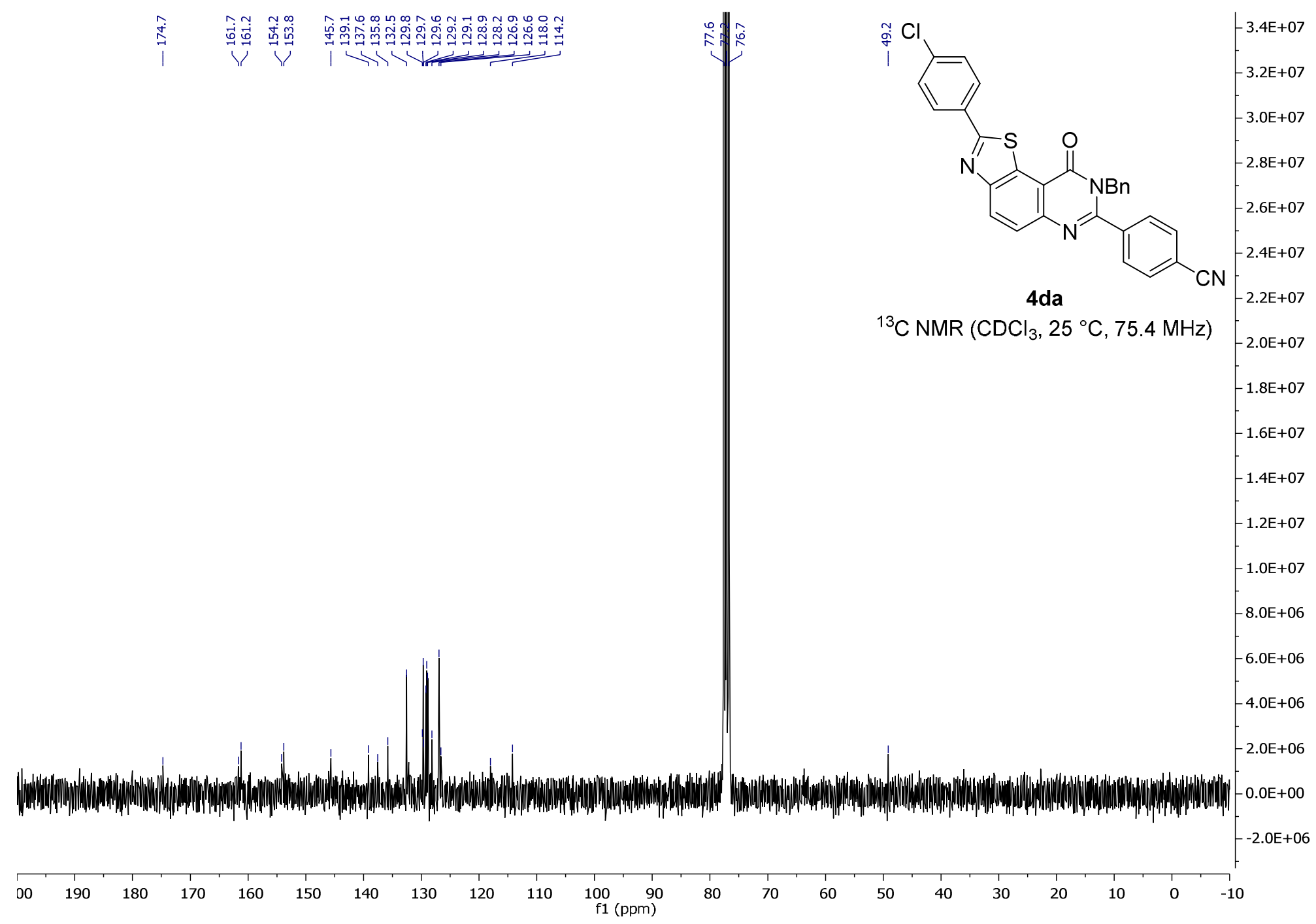


$N^{8}$-benzyl-2-(4-chlorophenyl)-7-( $p$-tolyl)thiazolo[5,4-f]quinazolin-9(8H)-one (4db)

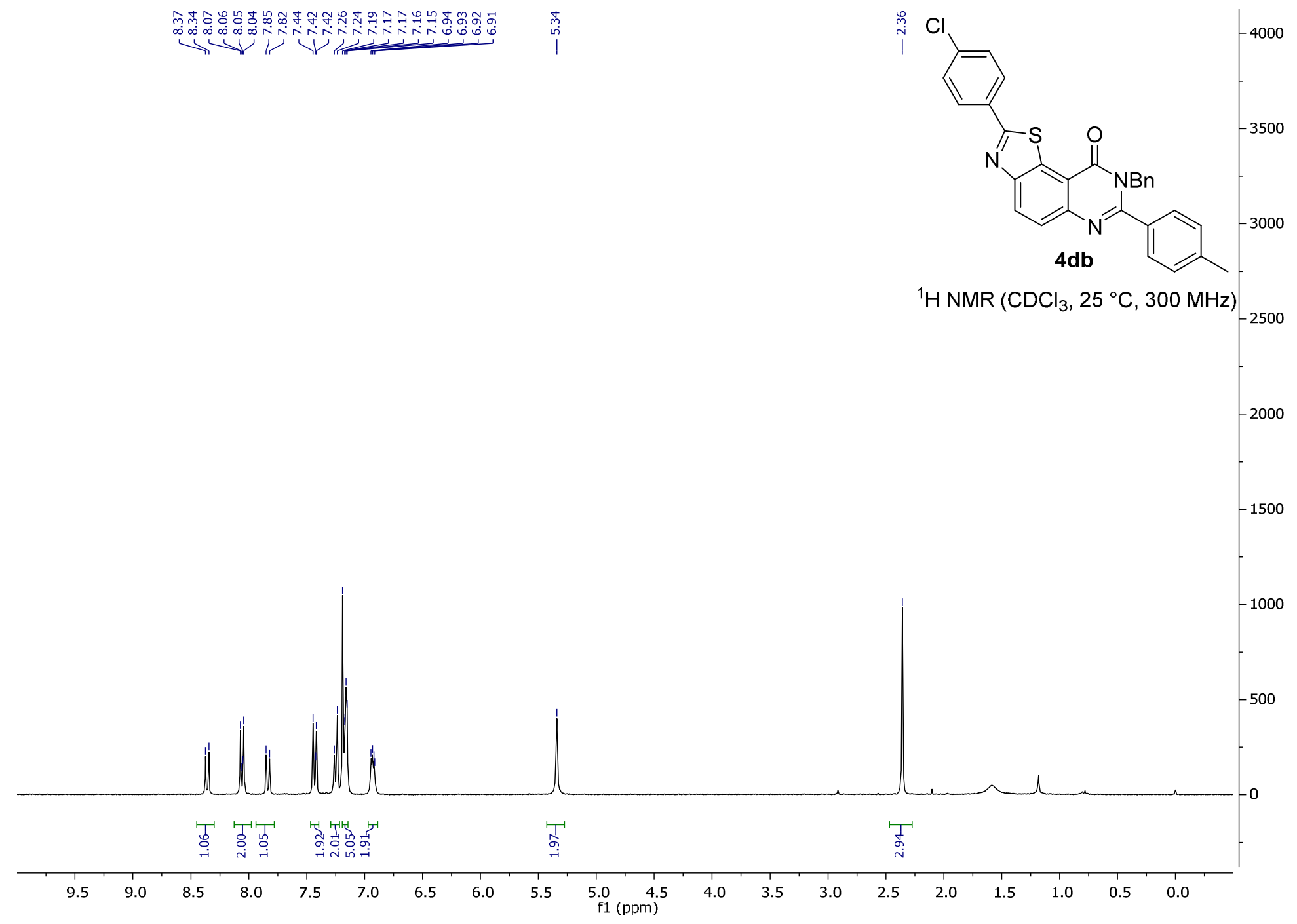




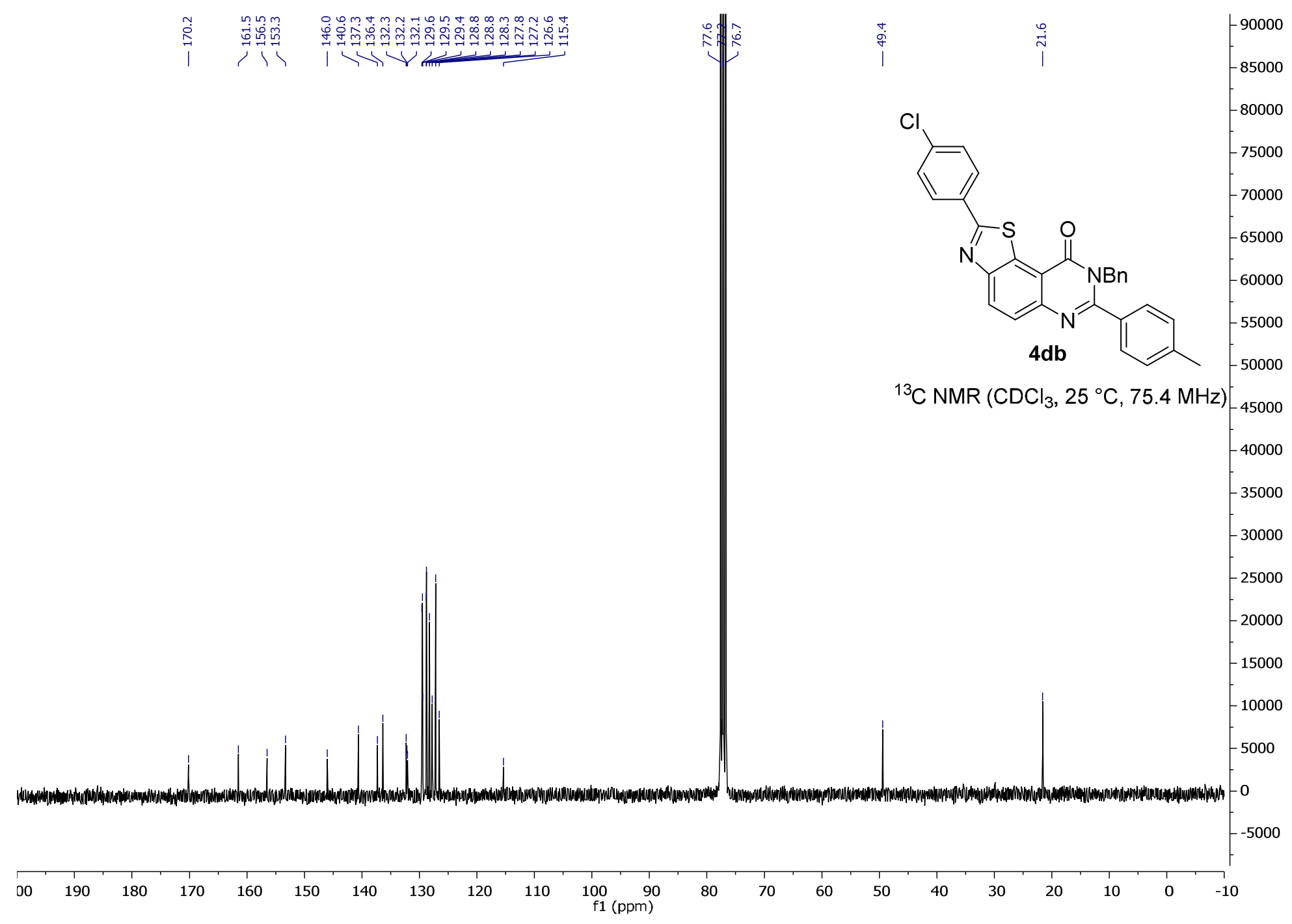


$N^{8}$-benzyl-2-(4-chlorophenyl)-7-(4-methoxyphenyl)thiazolo[5,4-f]quinazolin-9(8H)-one (4dc)

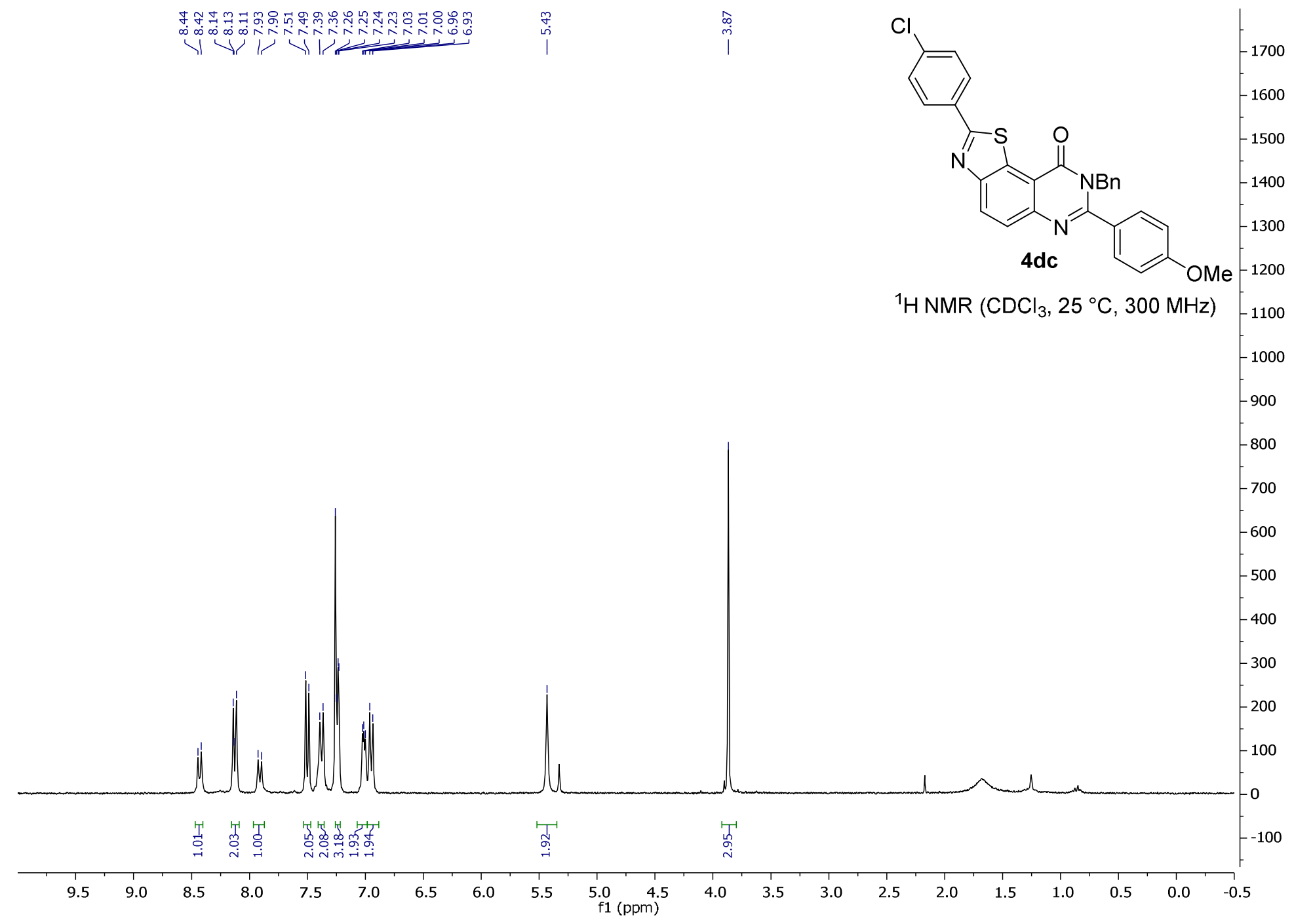



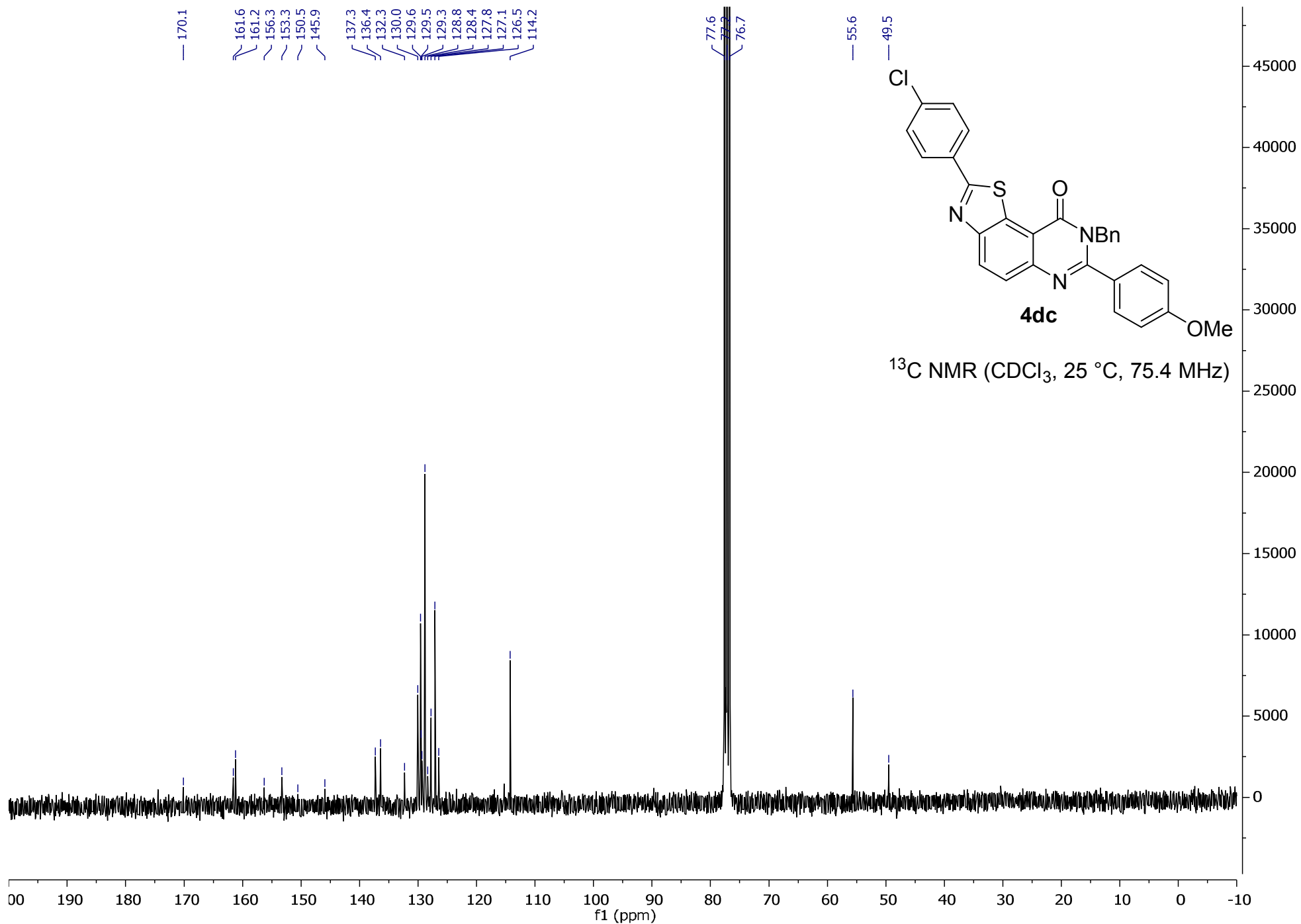
$N^{8}$-benzyl-2-(4-fluorophenyl)-7-(p-tolyl)thiazolo[5,4-f]quinazolin-9(8H)-one (4e)

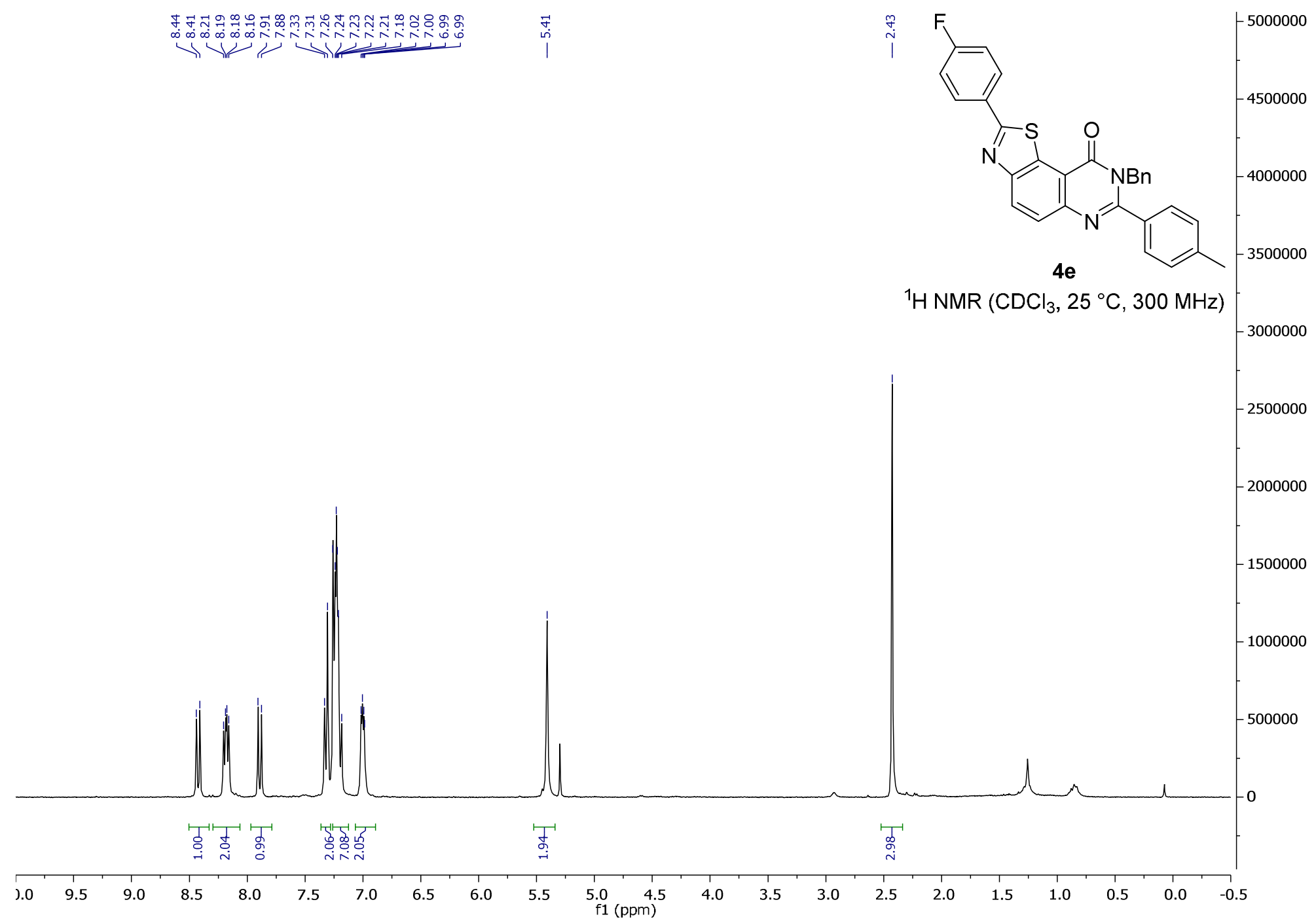




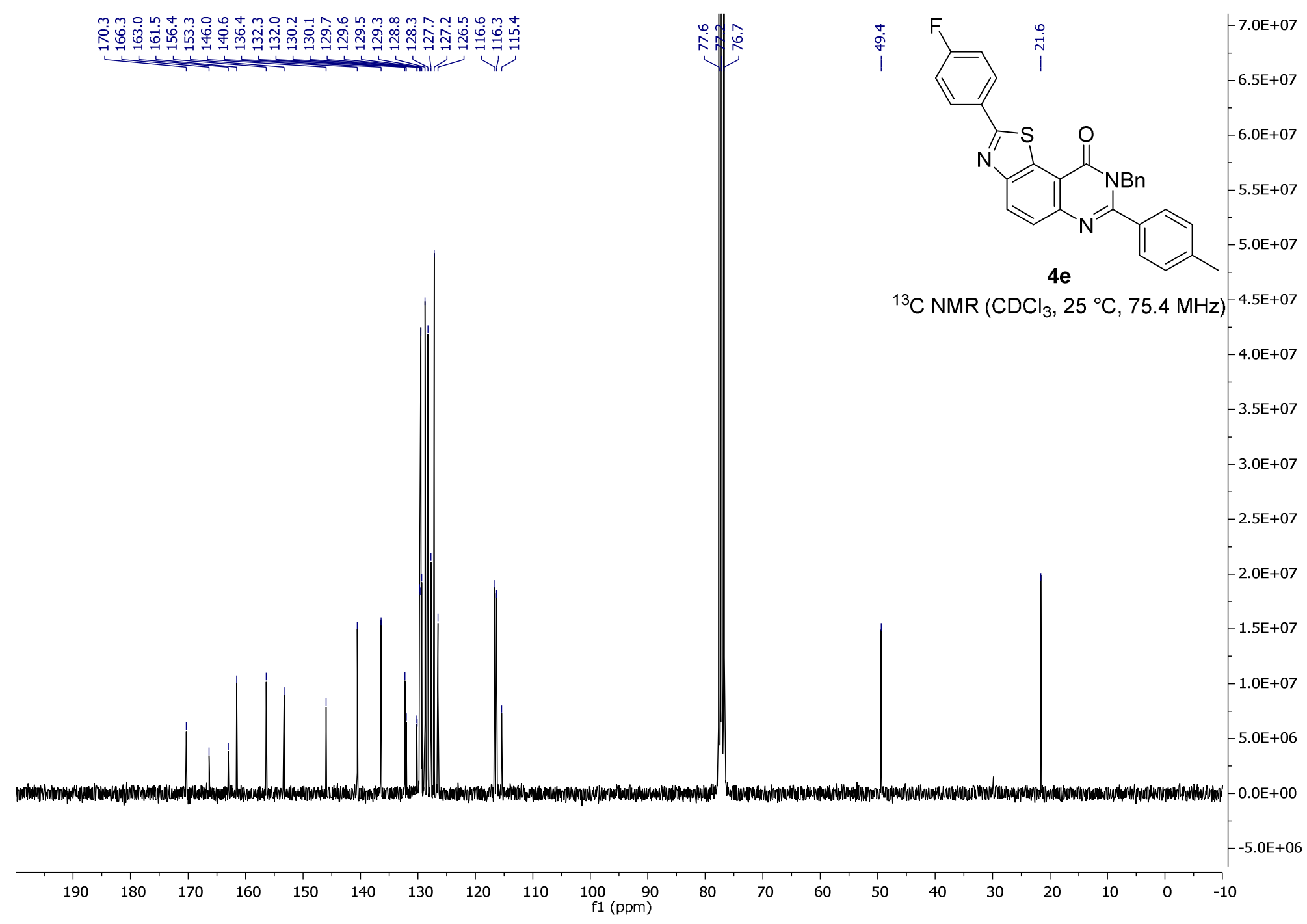


4-( $N^{8}$-benzyl-7-(4-fluorophenyl)-9-oxo-8,9-dihydrothiazolo[5,4-f]quinazolin-2-yl)benzonitrile (4fa)

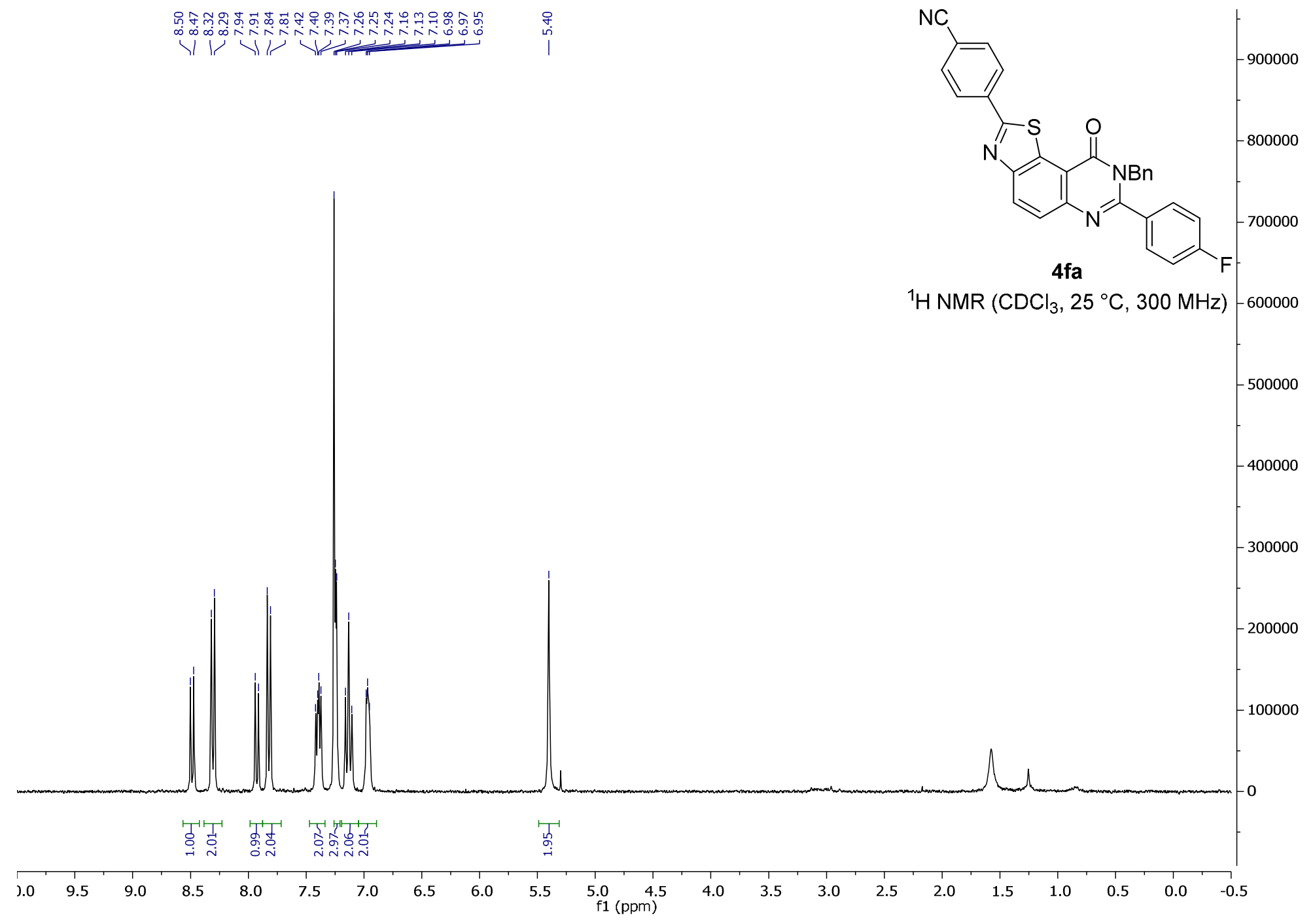




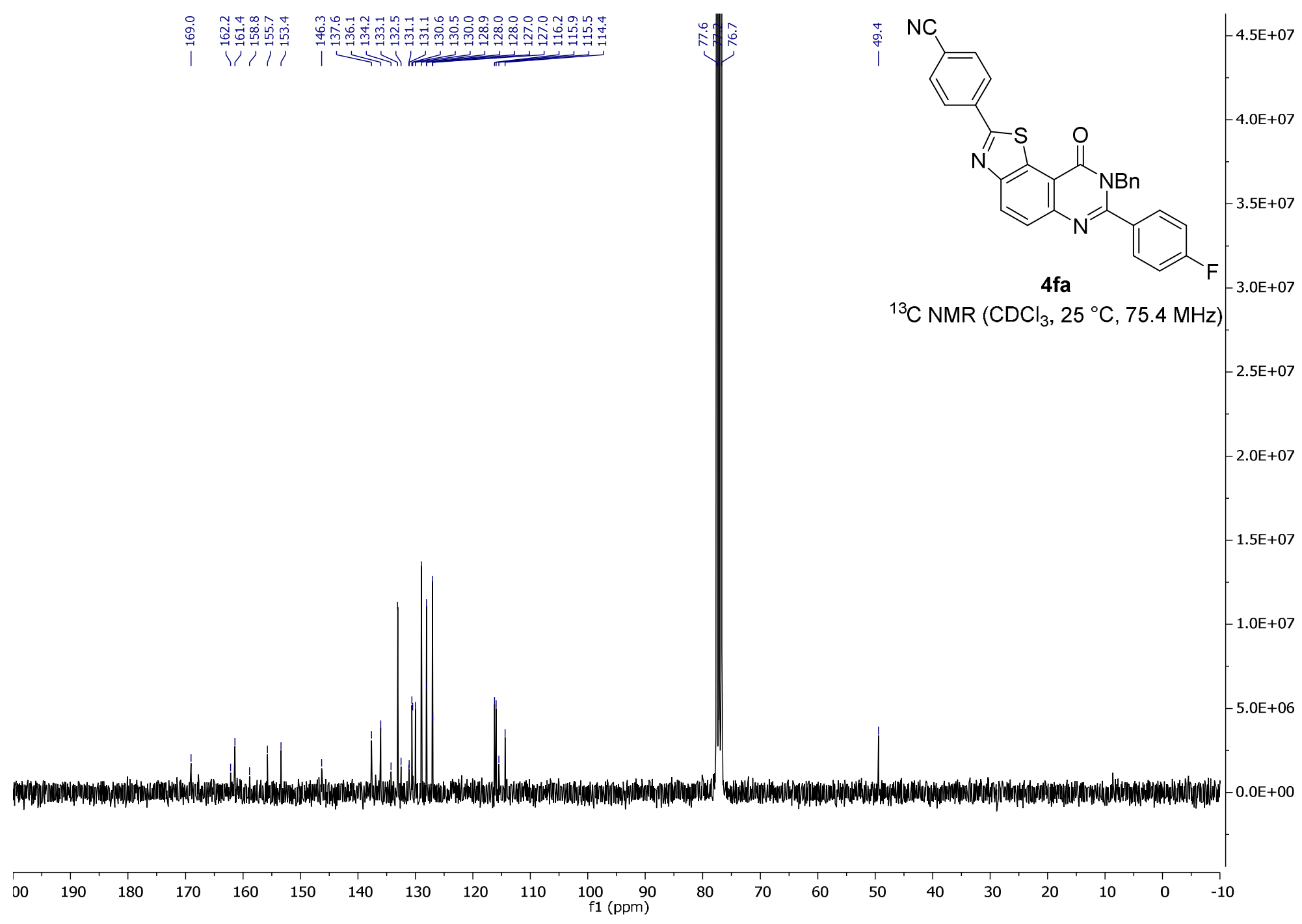


4-( $N^{8}$-benzyl-9 -oxo-7-(o-tolyl)-8,9-dihydrothiazolo[5,4-f] quinazolin-2-yl)benzonitrile (4fb)

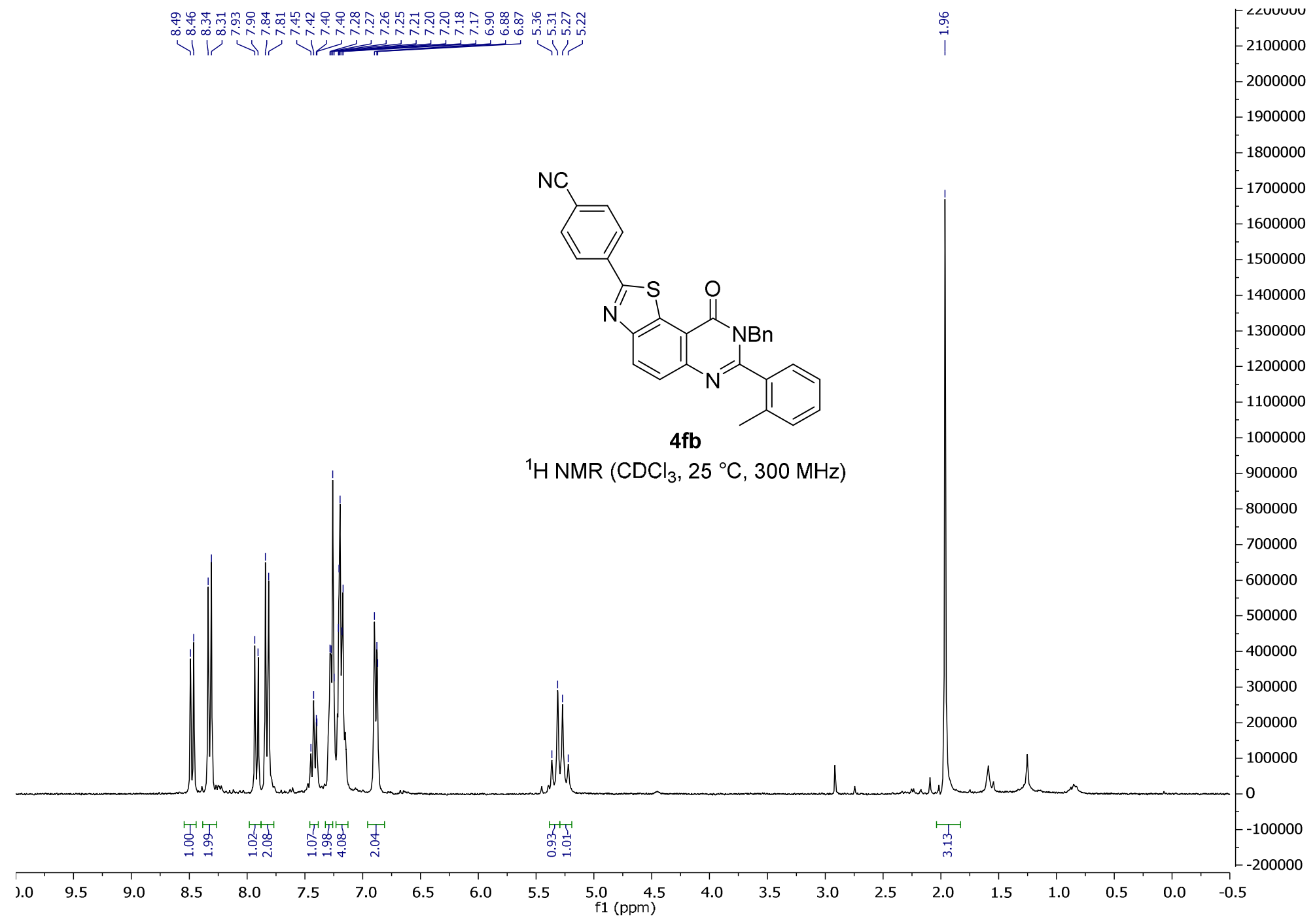




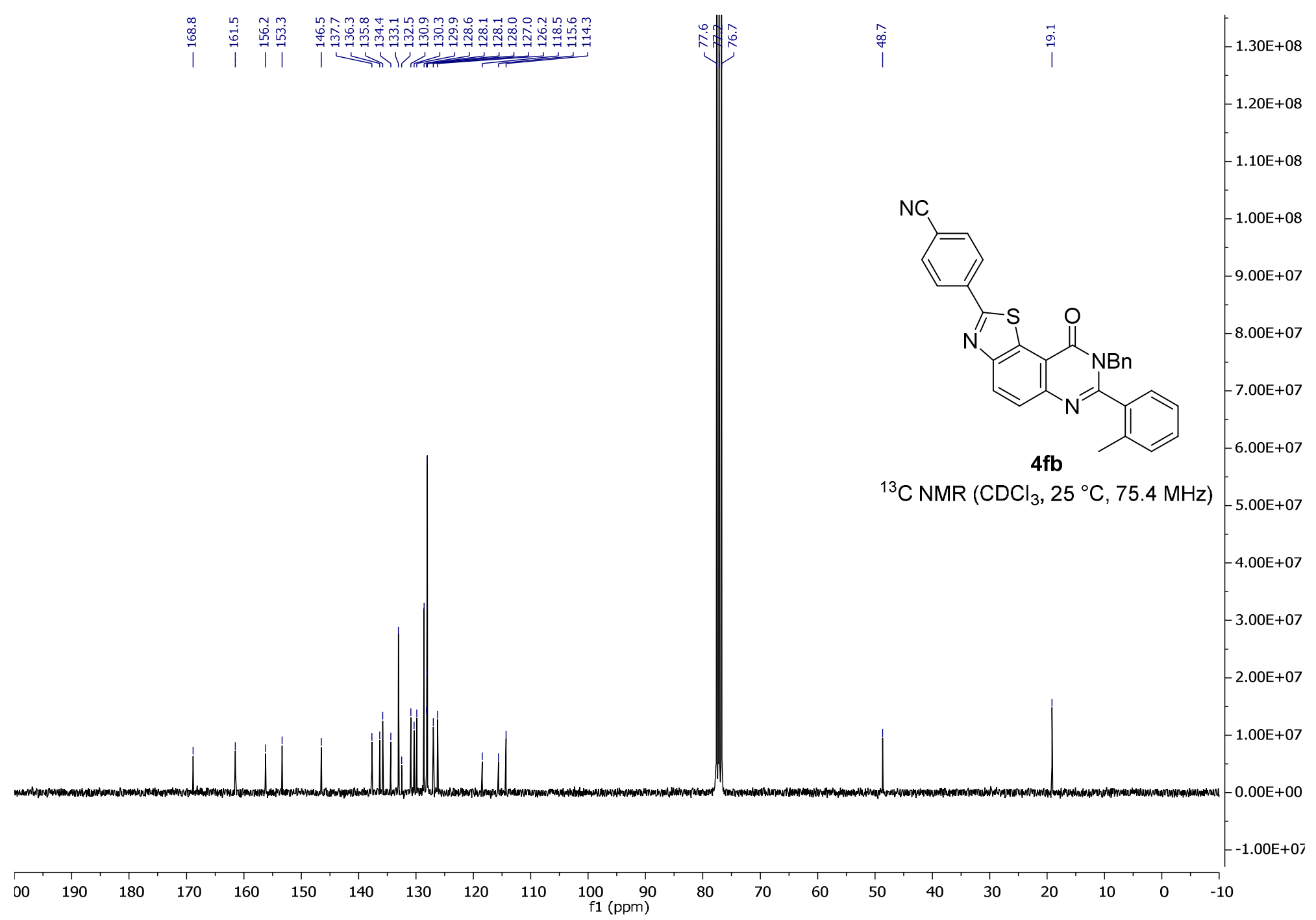

